\title{
Aromaticity and Möbius Antiaromaticity in Monocyclic [11]Annulenium Cations
}

\author{
Philip M. Warner \\ Department of Chemistry and Chemical Biology, Northeastern University, Boston, MA \\ 02115
}

\section{SUPPORTING INFORMATION}

Table of Contents

I. Coordinates and energies

1. Indene (11)

2. Isoindene (12)

3. [11]Annulenium, all cis planar-indene cation (13, NImag=3)

4. [11]Annulenium, all cis planar-isoindene cation $(\mathbf{1 4}, \mathrm{NImag}=3)$

5. Tropylium ion (15)

6. (RR/SS)-Hepta-3-enyl cation (16a1, diethyl allyl)

7. (RR/SS)-Octa-3-enyl cation (16a2, ethyl propyl allyl)

8. (RR/SS)-Nona-4-enyl cation (16a3, dipropyl allyl)

9. (RR/SS)-Nona-3,5-dienyl cation (16b1, diethyl pentadienyl)

10. (RR/SS)-Deca-3,5-dienyl cation (16b2, ethyl propyl pentadienyl)

11. (RR/SS)-Undeca-4,6-dienyl cation (16b3, dipropyl pentadienyl)

12. (RR/SS)-Undeca-3,5,7-trienyl cation (16c1, diethyl heptatrienyl)

13. (RR/SS)-Dodeca-3,5,7-trienyl cation (16c2, ethyl propyl heptatrienyl)

14. (RR/SS)-Trideca-4,6,8-trienyl_cation (16c3, dipropyl heptatrienyl)

15. (RR/SS)-Trideca-3,5,7,9-tetraenyl cation (16d1, diethyl nonatetraenyl)

16. (RR/SS)-Tetradeca-3,5,7,9-tetraenyl cation (16d2, ethyl propyl nonatetraenyl)

17. (RR/SS)-Pentadeca-4,6,8,10-tetraenyl cation (16d3, dipropyl nonatetraenyl)

18. (RR/SS)-Pentadeca-3,5,7,9,11-pentaenyl cation (16e1, diethyl undecapentaenyl)

19. (RR/SS)-Hexadeca-3,5,7,9,11-pentaenyl cation (16e2, ethyl propyl undecapentaenyl)

20. (RR/SS)-Heptadeca-4,6,8,10,12-pentaenyl cation (16e3, dipropyl undecapentaenyl)

21. (RR/SS)-Hex-3-ene (17a1, diethyl ethene)

22. (RR/SS)-Hept-3-ene (17a2, ethyl propyl ethene)

23. (RR/SS)-Oct-4-ene (17a3, dipropyl ethene)

24. (RR/SS)-Octa-3,5-diene (17b1, diethyl butadiene)

25. (RS/SR)-Octa-3,5-diene (iso-17b1, diethyl butadiene)

26. (RR/SS)-Nona-3,5-diene (17b2, ethyl propyl butadiene)

27. (RR/SS)-Deca-4,6-diene (17b3, dipropyl butadiene)

28. (RR/SS)-Deca-3,5,7-triene (17c1, diethyl hexatriene)

29. (RR/SS)-Undeca-3,5,7-triene (17c2, ethyl propyl hexatriene)

30. (RR/SS)-Dodeca-4,6,8-triene (17c3, dipropyl hexatriene)

31. (RR/SS)-Dodeca-3,5,7,9-tetraene (17d1, diethyl octatetraene)

32. (RR/SS)-Trideca-3,5,7,9-tetraene (17d2, ethyl propyl octatetraene)

33. (RR/SS)-Tetradeca-4,6,8,10-tetraene (17d3, dipropyl octatetraene)

34. (RR/SS)-Tetradeca-3,5,7,9,11-pentaene (17e1, diethyl decapentaene)

35. (RR/SS)-Pentadeca-3,5,7,9,11-pentaene (17e2, ethyl propyl decapentaene)

36. (RR/SS)-Hexadeca-4,6,8,10,12-pentaene (17e3, dipropyl decapentaene)

37. Bicyclo[4.3.0]nona-1(6),2,8-triene, held planar (NImag=1) (18)

38. Bicyclo[4.3.0]nona-2,6,9-triene, held planar (NImag=1) (19)

39. Tropylium-indene cation $(\mathbf{2 0})$

40. Tropylium-isoindene cation (21)

41. 7,8-Dihydrotropylium-indene cation, held planar (NImag=1) (22)

42. 7,8-Dihydro-2H-tropylium-isoindene cation, held planar $(\mathrm{NImag}=1)(\mathbf{2 3})$ 
43. 11,12-Dihydro[11] annulenium mono-trans planar-indene cation (NImag=3) (24) S32

44. 11,12-Dihydro-2H[11] annulenium mono-trans planar-isoindene cation (NImag=3) (25) $\quad$ S32

45. [11]Annulenium cation, di-trans, 5+4 type (26) $\quad$ S33

46. [11]Annulenium cation, di-trans, 6+3 type (27) S34

47. [11]Annulenium cation, tri-trans (28) S34

48. [11]Annulenium cation, mono-trans tub (29)

49. [11]Annulenium cation, mono-trans planar (NImag $=2: 52 \mathrm{i}, 95 \mathrm{i})(\mathbf{3 0}) \quad \mathrm{S} 35$

50. [11]Annulenium cation, all cis $\mathrm{C}_{2}(\mathbf{3 1}) \quad \mathrm{S} 36$

51. [11]Annulenium cation, all cis $\mathrm{C}_{\mathrm{s}}$ tub (NImag=1: 19i) (32) S37

52. [11]Trannulenium cation $\left(\mathrm{C}_{\mathrm{s}}\right)(\mathbf{3 3}) \quad \mathrm{S} 37$

53. [11]Annulenium cation, all cis $\mathrm{D}_{11 \mathrm{~h}}$ planar (NImag=2: 119.1i, 119.5i) (34) S38

54. [11]Annulenium cation triplet, di-trans 5+4 type (26t) S38

55. [11]Annulenium cation triplet, di-trans $6+3$ type (27t) $\quad$ S39

56. [11]Annulenium cation triplet, tri-trans $(\mathbf{2 8 t}) \quad$ S39

57. [11]Annulenium cation triplet, mono-trans (29t) $\quad \mathrm{S} 40$

58. [11]Annulenium cation triplet, all cis (31t) $\quad \mathrm{S} 41$

59. [11]Trannulenium cation triplet $\left(\mathrm{C}_{2}\right)(\mathbf{3 3 t}) \quad \mathrm{S} 41$

60. [11]Annulenium, di-trans, $5+4$ type-indene cation (35) S42

61. [11]Annulenium, di-trans, 5+4 type-isoindene cation (36) $\quad \mathrm{S} 42$

62. [11]Annulenium, di-trans, $6+3$ type-indene cation (37) $\quad$ S43

63. [11]Annulenium, di-trans, $6+3$ type-isoindene cation (38) $\quad \mathrm{S} 44$

64. [11]Annulenium, tri-trans-indene cation (39) $\quad$ S44

65. [11]Annulenium, tri-trans-isoindene cation (40)

66. [11]Annulenium, mono-trans tub-indene cation (41) S46

67. [11]Annulenium, mono-trans tub-isoindene cation (42) $\quad \mathrm{S} 47$

68. [11]Annulenium, mono-trans planar-indene cation (43, NImag=2) $\quad$ S47

69. [11]Annulenium, mono-trans planar-isoindene cation (44, NImag=2) $\quad$ S48

70. [11]Annulenium, all cis-indene cation (45) $\quad$ S49

71. [11]Annulenium, all cis-isoindene cation (46) $\quad$ S49

72. [11]Annulenium, all cis-indene cation isomer (47) $\quad$ S50

73. [11]Annulenium, all cis tub-indene cation $(\mathbf{4 8}, \mathrm{NImag}=1) \quad \mathrm{S}$ (1)

74. [11]Annulenium, all cis tub-isoindene cation $(\mathbf{4 9}, \mathrm{NImag}=1) \quad$ S51

75. [11]Trannulenium-indene cation (50) S52

76. [11]Trannulenium-isoindene cation (51) S53

77. Cyclopropa[1,2][11]annulenium cation, planar (NImag=2: 95i, 100i) (52a) S53

78. Cyclopropa[1,2][11]annulenium cation, non-planar (52b) $\quad$ S54

79. Cyclopropa[1,2][11]annulenium cation triplet, non-planar (52t) S54

80. Dicyclopropa[1,2:6,7][11] annulenium cation, planar (NImag=2: 48.0i, 48.4i) (53a) S55

81. Dicyclopropa[1,2:6,7][11] annulenium cation, non-planar (53b) S56

82. Tricyclopropa[1,2:4,5:8,9][11] annulenium cation, planar (54) S56

83. Tetracyclopropa[1,2:3,4:6,7:8,9][11]annulenium cation, planar (55) S57

84. Pentacyclopropa[1,2:3,4:5,6:7,8:9,10][11]annulenium cation, planar (56) S58

85. Cyclopropa[1,2][10]annulene, planar (NImag=1: 15i) (57) S58

86. Cyclopropa[1,2][10]annulene, non-planar (57a) S59

87. Tricyclopropa[1,2:4,5:7,8][10]annulene, planar (58) S59

88. Tetracyclopropa[1,2:3,4:6,7:8,9][10]annulene, planar (59) S60

89. Tricyclopropa[1,2:5,6:9,10][12]annulene, planar $(\mathbf{6 0}) \quad$ S61

90. Tricyclopropa[1,2:5,6:9,10][12]annulene triplet, planar (NImag=2: $5 \mathrm{i}, 14 \mathrm{i})(\mathbf{6 0 t}-\mathbf{a}) \quad \mathrm{S} 61$

91. Tricyclopropa[1,2:5,6:9,10][12]annulene triplet, non-planar (60t-b) S62

92. Heptacyclopropa[15]annulenium cation, planar (NImag=2: 10i, 14i) (61) S62

93. Heptacyclopropa[15]annulenium cation, non-planar (61a) S63

94. 1;2,6;7-Bisdehydro[11]annulenium cation (62) $\quad$ S64

95. Dicyclopropa[1,2:4,5][11] annulenium cation, non-planar (63) $\quad$ S65

96. Dicyclopropa[1,2:4,5][11] annulenium cation triplet, non-planar (63t) S65

97. Dicyclopropa[1,2:3,4][11] annulenium cation, non-planar (64) S66

98. Dicyclopropa[1,2:3,4][11]annulenium cation triplet, non-planar (64t) S66 
99. 1,2-Dihydrobenzene, held planar (NImag=1) $\quad$ S67

100. 1,2-Dihydrotropylium cation, held planar (NImag=1) $\quad$ S68

101. 1,2-Dihydro[11] annulenium cation, all cis, held planar (NImag=4) $\quad$ S68

102. [11]Annulenium, di-trans, $5+4$ type-indene cation, isomer of $\mathbf{3 5} \quad S 69$

103. [11]Annulenium, di-trans, $5+4$ type-indene cation, another isomer of $\mathbf{3 5} \quad S 69$

104. [11]Annulenium, di-trans, 5+4 type-isoindene cation, isomer of $\mathbf{3 6} \quad \mathrm{S} 70$

105. [11]Annulenium, all cis-indene cation, another isomer of $\mathbf{4 5} \quad$ S71

106. [11]Annulenium, all cis-indene cation, another isomer of $\mathbf{4 5} \quad \mathrm{S} 72$

107. [11]Annulenium, all cis-isoindene cation, isomer of $\mathbf{4 6} \quad \mathrm{S} 73$

108. [11]Annulenium, all cis-indene cation, another isomer of $\mathbf{4 5} \quad \mathrm{S} 73$

109. [11]Annulenium, all cis-indene cation, another isomer of $\mathbf{4 5} \quad \mathrm{S} 74$

110. [11]Annulenium, all cis-indene cation, another isomer of $\mathbf{4 6} \quad \mathbf{S 7 5}$

$\begin{array}{ll}\text { II. Table SI1. Summary Data for Equation } 6 . & \text { S76 }\end{array}$

I. Coordinates and energies for all new species discussed. Unless noted otherwise, all species are minima $(\mathrm{NImag}=0)$ by frequency analysis.

1. Indene (11)

$\mathrm{C}, 0,-0.2268344519,0.0000110587,-0.7180927872$

$\mathrm{C}, 0,-0.2156446402,-0.000057815,0.6941296852$

C, $0,0.9852922499,0.0000386431,1.3909244593$

C, $0,2.1866180945,0.0002071346,0.6690559586$

C, $0,2.1768854139,0.0002777359,-0.7293909041$

$\mathrm{C}, 0,0.9704544011,0.0001791969,-1.4358504271$

C, $0,-1.6243038572,-0.0001169824,-1.1638046748$

C, $0,-2.4434421726,-0.0002720378,-0.0943711038$

C, $0,-1.6456959134,-0.0002414056,1.1899772941$

$\mathrm{H}, 0,1.0010290819,-0.0000169881,2.4786045473$

$\mathrm{H}, 0,3.1341308031,0.0002841257,1.2013604614$

$\mathrm{H}, 0,3.1181128944,0.0004100724,-1.2731248804$

$\mathrm{H}, 0,0.9685819037,0.000236756,-2.5231498884$

$\mathrm{H}, 0,-1.9323083562,-0.0000966047,-2.204652644$

$\mathrm{H}, 0,-1.8710081976,0.8782103767,1.8128502452$

$\mathrm{H}, 0,-1.8708172122,-0.8787821379,1.8127916763$

$\mathrm{H}, 0,-3.5276956611,-0.0003987699,-0.1201445183$

$\mathrm{E}\left(\mathrm{B} 3 \mathrm{LYP} / 6-31 \mathrm{G}^{*} \mathrm{opt}\right)=$

$-347.762854$

Sum of electronic and zero-point Energies $=\quad-347.621927$

Sum of electronic and thermal Energies $=\quad-347.615554$

Sum of electronic and thermal Enthalpies= $\quad-347.614610$

Sum of electronic and thermal Free Energies $=\quad-347.652389$

$\mathrm{E}\left(\mathrm{B} 3 \mathrm{LYP} / 6-311+\mathrm{G}^{* *} / / \mathrm{B} 3 \mathrm{LYP} / 6-31 \mathrm{G}^{*}\right)=\quad-347.850977$

$\chi=-74.3$

2. Isoindene (12)

C, $0,-0.254261091,0.0000080995,-0.7421170625$

C, $0,-0.2543293699,-0.0000616606,0.742094372$

C, $0,1.0076466342,0.0000460639,1.4419409745$

$\mathrm{C}, 0,2.1636271178,0.0002082476,0.7260650516$

C, $0,2.1636935994,0.0002763719,-0.7258670951$

C, $0,1.0077783384,0.0001819088,-1.4418487562$

C, $0,-1.5415331795,-0.0001139906,-1.1865003438$ 
C, $0,-2.462601329,-0.0002800798,-0.0001139692$

C, $0,-1.5416416198,-0.0002263888,1.1863596766$

$\mathrm{H}, 0,1.0207798689,-0.0000033852,2.5290375495$

$\mathrm{H}, 0,3.1216468896,0.000290722,1.2396584064$

$\mathrm{H}, 0,3.1217604055,0.0004067439,-1.2393725569$

$\mathrm{H}, 0,1.0210101799,0.0002347039,-2.5289440932$

$\mathrm{H}, 0,-1.8757993973,-0.000102907,-2.2175280078$

$\mathrm{H}, 0,-3.1317118943,-0.876325946,-0.0001838993$

$\mathrm{H}, 0,-1.876006394,-0.0003098751,2.2173550763$

$\mathrm{H}, 0,-3.1319542617,0.8755785124,-0.0000995628$

$\mathrm{E}\left(\mathrm{B} 3 \mathrm{LYP} / 6-31 \mathrm{G}^{*}\right.$ opt $)=$

$-347.726815$

Sum of electronic and zero-point Energies $=\quad-347.587242$

Sum of electronic and thermal Energies $=\quad-347.580668$

Sum of electronic and thermal Enthalpies= $\quad-347.579724$

Sum of electronic and thermal Free Energies $=\quad-347.617846$

$\mathrm{E}\left(\mathrm{B} 3 \mathrm{LYP} / 6-311+\mathrm{G}^{* *} / / \mathrm{B} 3 \mathrm{LYP} / 6-31 \mathrm{G}^{*}\right)=\quad-347.815884$

$\chi=-64.9$

3. [11]Annulenium, all cis planar-indene cation (13, NImag=3)

$\begin{array}{rrrr}6 & 3.444329 & -0.028035 & 0.000075 \\ 6 & 3.023149 & -1.358948 & -0.000084 \\ 6 & 1.923130 & -2.241910 & -0.000261 \\ 6 & 0.532156 & -2.403032 & -0.000398 \\ 6 & -0.759288 & -1.822913 & -0.000457 \\ 6 & -1.585756 & -0.692279 & -0.000425 \\ 6 & -1.589869 & 0.749421 & -0.000292 \\ 6 & -0.723235 & 1.879669 & -0.000113 \\ 6 & 0.569187 & 2.413304 & 0.000049 \\ 6 & 1.972122 & 2.220225 & 0.000154 \\ 6 & 3.042906 & 1.323426 & 0.000165 \\ 1 & 4.532387 & -0.034760 & 0.000412 \\ 1 & 3.918624 & -1.976876 & 0.000268 \\ 1 & 2.343361 & -3.245430 & 0.000159 \\ 1 & 0.362556 & -3.477159 & 0.000056 \\ 1 & -1.432424 & -2.677891 & 0.000464 \\ 1 & -1.379326 & 2.744920 & 0.000916 \\ 1 & 0.434564 & 3.492593 & 0.000807 \\ 1 & 2.407683 & 3.216947 & 0.000733 \\ 1 & 3.953416 & 1.919101 & 0.000659 \\ 6 & -3.001465 & 1.165064 & -0.000881 \\ 6 & -3.060724 & -1.128095 & 0.001086 \\ 6 & -3.847032 & 0.120224 & 0.000248 \\ 1 & -3.294091 & 2.208903 & -0.002494 \\ 1 & -4.929636 & 0.165803 & -0.000123 \\ 1 & -3.277909 & -1.758103 & -0.875020 \\ 1 & -3.276862 & -1.754781 & 0.879964\end{array}$

$\mathrm{E}\left(\mathrm{B} 3 \mathrm{LYP} / 6-31 \mathrm{G}^{*}\right.$ opt $)=$

$-540.876309$

Sum of electronic and zero-point Energies $=\quad-540.648578$

Sum of electronic and thermal Energies $=\quad-540.639051$

Sum of electronic and thermal Enthalpies= $\quad-540.638107$

Sum of electronic and thermal Free Energies $=\quad-540.683829$

$\mathrm{E}\left(\mathrm{B} 3 \mathrm{LYP} / 6-311+\mathrm{G}^{* *} / / \mathrm{B} 3 \mathrm{LYP} / 6-31 \mathrm{G}^{*}\right)=\quad-541.004643$

$\chi=-148.0$ 
4. [11]Annulenium, all cis planar-isoindene cation (14, NImag=3)

$\begin{array}{lrrr}6 & -3.426044 & -0.000052 & -0.000012 \\ 6 & -3.031408 & -1.351154 & -0.000014 \\ 6 & -1.953843 & -2.238786 & -0.000006 \\ 6 & -0.544321 & -2.450436 & 0.000009 \\ 6 & 0.729868 & -1.908665 & 0.000027 \\ 6 & 1.611581 & -0.759445 & 0.000042 \\ 6 & 1.611569 & 0.759454 & 0.000049 \\ 6 & 0.729761 & 1.908662 & 0.000045 \\ 6 & -0.544364 & 2.450445 & 0.000033 \\ 6 & -1.953949 & 2.238788 & 0.000015 \\ 6 & -3.031416 & 1.351106 & -0.000001 \\ 1 & -4.514905 & -0.000051 & -0.000039 \\ 1 & -3.940227 & -1.947848 & -0.000077 \\ 1 & -2.390182 & -3.236071 & -0.000052 \\ 1 & -0.415327 & -3.529710 & -0.000008 \\ 1 & 1.399790 & -2.763622 & -0.000008 \\ 1 & 1.399752 & 2.763581 & -0.000023 \\ 1 & -0.415368 & 3.529717 & -0.000014 \\ 1 & -2.390312 & 3.236059 & -0.000012 \\ 1 & -3.940266 & 1.947759 & -0.000040 \\ 6 & 2.936581 & 1.148342 & -0.000129 \\ 6 & 2.936644 & -1.148263 & -0.000006 \\ 6 & 3.860363 & 0.000020 & 0.000049 \\ 1 & 3.276370 & -2.176762 & -0.000086 \\ 1 & 3.276336 & 2.176835 & -0.000284 \\ 1 & 4.534124 & 0.000104 & 0.873489 \\ 1 & 4.534081 & -0.000089 & -0.873447\end{array}$

$\mathrm{E}\left(\mathrm{B} 3 \mathrm{LYP} / 6-31 \mathrm{G}^{*} \mathrm{opt}\right)=$

$-540.847950$

Sum of electronic and zero-point Energies= Sum of electronic and thermal Energies= Sum of electronic and thermal Enthalpies= Sum of electronic and thermal Free Energies= $-540.621131$ $-540.611544$ $-540.610600$ $-540.656204$ $\mathrm{E}\left(\mathrm{B} 3 \mathrm{LYP} / 6-311+\mathrm{G}^{* *} / / \mathrm{B} 3 \mathrm{LYP} / 6-31 \mathrm{G}^{*}\right)=$ $-540.976963$ $\chi=-51.1$

5. Tropylium ion (15)

C,0,-1.4524955062,-0.0000129228,-0.6994692259 C, $0,-1.4524954412,-0.0000305796,0.699469527$ C, $0,-0.3587426585,-0.0000241521,1.5717129961$ C, $0,-0.3587428703,0.0000220878,-1.5717129125$ C, $0,1.0051473955,-0.000011871,1.260401222$ C, $0,1.0051472012,0.0000505391,-1.2604014803$ C, $0,1.6121936423,0.0000064039,-0.0000001266$ $\mathrm{H}, 0,-2.4324409434,-0.0000284744,-1.1714137789$ $\mathrm{H}, 0,-2.4324408157,-0.0000480412,1.1714141988$ $\mathrm{H}, 0,-0.6008197227,-0.000034331,2.6321028475$ $\mathrm{H}, 0,-0.6008202122,0.0000387275,-2.6321027058$ $\mathrm{H}, 0,1.683295093,-0.0000275424,2.1107729567$ $\mathrm{H}, 0,1.6832948495,0.0001104325,-2.1107732411$ H,0,2.6998611745,-0.0000078022,-0.0000002755 
$\mathrm{E}\left(\mathrm{B} 3 \mathrm{LYP} / 6-31 \mathrm{G}^{*}\right.$ opt $)=$

Sum of electronic and zero-point Energies= Sum of electronic and thermal Energies= Sum of electronic and thermal Enthalpies= Sum of electronic and thermal Free Energies= $\mathrm{E}\left(\mathrm{B} 3 \mathrm{~L} Y \mathrm{P} / 6-311+\mathrm{G} * * / / \mathrm{B} 3 \mathrm{LYP} / 6-31 \mathrm{G}^{*}\right)=$
$-270.678568$

$-270.559094$

$-270.553656$

$-270.552712$

$-270.588130$

$-270.740207$

6. (RR/SS)-Hepta-3-enyl cation (16a1, diethyl allyl)

(Note: for this and related compounds, RR/SS = anti conformation; RS/SR = syn conformation; these stereoisomers are isoenergetic)

C, $0,-2.5559426764,0.0486180061,0.1468100303$

$\mathrm{H}, 0,-2.5751621544,0.068844609,1.2400169769$

$\mathrm{C}, 0,-1.181144303,0.0424539181,-0.3697426132$

$\mathrm{H}, 0,-1.0692518928,0.0630134242,-1.4561503936$

$\mathrm{C}, 0,-0.0248585972,-0.0078634455,0.3955631753$

$\mathrm{H}, 0,-0.091884319,-0.0325359267,1.480962505$

C,0,1.2169252573,-0.027185581,-0.2232100377

$\mathrm{H}, 0,1.2395945048,0.0020504793,-1.3149454746$

C, $0,2.5176916108,-0.0620720321,0.4576772675$

$\mathrm{H}, 0,3.0396272638,-0.9701886879,0.1101505823$

C,0,3.4022039851,1.1574680851,0.0581527645

$\mathrm{H}, 0,2.9503273135,2.0951028925,0.3914519168$

$\mathrm{H}, 0,3.5594328693,1.202315319,-1.0234592393$

H,0,2.4022389359,-0.1300032987, 1.5430133227

H, $0,4.3751625113,1.048783259,0.5434279593$

$\mathrm{H}, 0,-3.0354150315,0.9713637812,-0.2225244193$

C, $0,-3.378606461,-1.1517883432,-0.411222296$

H,0,-4.4047714297,-1.0632092552,-0.0464146009

$\mathrm{H}, 0,-3.4001581971,-1.1500724399,-1.504933351$

$\mathrm{H}, 0,-2.9673532672,-2.1032478016,-0.0647655287$

$\mathrm{E}\left(\mathrm{B} 3 \mathrm{LYP} / 6-31 \mathrm{G}^{*}\right.$ opt $)=$

Sum of electronic and zero-point Energies=

Sum of electronic and thermal Energies=

Sum of electronic and thermal Enthalpies=

Sum of electronic and thermal Free Energies= $\mathrm{E}\left(\mathrm{B} 3 \mathrm{LYP} / 6-311+\mathrm{G}^{* *} / / \mathrm{B} 3 \mathrm{LYP} / 6-31 \mathrm{G}^{*}\right)=$

$\chi=-56.3$

$\%$ of total Charge on $\pi$ system (Mulliken analysis) $=57.9 \%$

7. (RR/SS)-Octa-3-enyl cation (16a2, ethyl propyl allyl)

C,0,-1.9368752395,0.2977730224,0.1501081775

$\mathrm{H}, 0,-1.9463324085,0.2732223569,1.2435166329$

C, $0,-0.5757548683,0.3376696133,-0.3848993143$

$\mathrm{H}, 0,-0.4740485752,0.5201786327,-1.4568369011$

C, $0,0.5879228083,0.1127180333,0.3406819368$

$\mathrm{H}, 0,0.5314795841,-0.0745802804,1.4107965482$

C, $0,1.8192345445,0.1384592506,-0.2940814191$

$\mathrm{H}, 0,1.8317824614,0.3360832659,-1.3683205612$

C,0,3.1261032062,-0.0521074996,0.3517782145

$\mathrm{H}, 0,3.6048009698,-0.9174101074,-0.1375910356$

C,0,4.0571069555,1.1766332677,0.1310468972

$\mathrm{H}, 0,3.6478069742,2.0698892431,0.6098303438$

H,0,4.2065704956,1.3807885528,-0.9332706866
$-274.288762$

$-274.105818$

$-274.096471$

$-274.095527$

$-274.140869$

$-274.359940$ 
$\mathrm{H}, 0,3.0179616316,-0.281206565,1.4157281121$

$\mathrm{H}, 0,-2.485274655,1.1856314018,-0.1969124203$

C, $0,-2.7015688787,-0.9576741587,-0.4245259966$

$\mathrm{H}, 0,-2.6915543279,-0.913708645,-1.5193432602$

$\mathrm{H}, 0,-2.1634906951,-1.8641264326,-0.1274577832$

C,0,-4.1404639871,-0.9929925184,0.0976459025

$\mathrm{H}, 0,-4.7027638376,-0.1076317897,-0.2167050072$

$\mathrm{H}, 0,-4.6502528508,-1.8766612041,-0.2995319804$

H, $0,-4.1703075064,-1.0508303721,1.1905545459$

$\mathrm{H}, 0,5.0293954945,0.9574878799,0.5790170622$

$\mathrm{E}\left(\mathrm{B} 3 \mathrm{LYP} / 6-31 \mathrm{G}^{*}\right.$ opt $)=$

$-313.604846$

Sum of electronic and zero-point Energies=

Sum of electronic and thermal Energies=

$-313.393123$

$-313.382472$

$-313.381527$

Sum of electronic and thermal Free Energies=

$-313.430472$

$\mathrm{E}\left(\mathrm{B} 3 \mathrm{LYP} / 6-311+\mathrm{G}^{* *} / / \mathrm{B} 3 \mathrm{LYP} / 6-31 \mathrm{G}^{*}\right)=$

$-313.686495$

$\chi=-66.2$

$\%$ of total Charge on $\pi$ system (Mulliken analysis) $=56.1 \%$

8. (RR/SS)-Nona-4-enyl cation (16a3, dipropyl allyl)

C, $0,-2.5309436507,0.1897289549,-0.0197175756$

$\mathrm{H}, 0,-2.5461267878,0.3245933434,1.0657176597$

C, $0,-1.1645369691,0.1152844815,-0.5422338647$

$\mathrm{H}, 0,-1.0549303124,0.1196590602,-1.6288295517$

C, $0,-0.0098834264,-0.0096441318,0.2171929916$

$\mathrm{H}, 0,-0.0733179731,-0.0222007244,1.3031712301$

C, $0,1.2257383608,-0.1163504631,-0.4054020162$

$\mathrm{H}, 0,1.2433158119,-0.0955965391,-1.4971742949$

C, $0,2.5223796145,-0.1984269283,0.2709411701$

$\mathrm{H}, 0,3.0956753947,-1.0321233105,-0.1610181423$

C, $0,3.3572942511,1.110667629,0.0036992831$

$\mathrm{H}, 0,2.7998766303,1.9694879159,0.3927200485$

$\mathrm{H}, 0,3.465224027,1.2531732649,-1.0775807155$

$\mathrm{H}, 0,2.4120558845,-0.3612444574,1.3470070031$

$\mathrm{H}, 0,-3.044526693,1.0397096602,-0.4934031295$

C, $0,-3.3377951624,-1.1036784601,-0.4168514883$

$\mathrm{H}, 0,-3.3124745533,-1.2224871773,-1.5060681827$

$\mathrm{H}, 0,-2.8400634064,-1.9766054157,0.0188768545$

C, $0,-4.7840161765,-1.0096152428,0.0767821471$

$\mathrm{H}, 0,-5.3068043731,-0.1606759138,-0.3760036621$

$\mathrm{H}, 0,-5.3233984959,-1.9228572187,-0.194124218$

H,0,-4.8312817033,-0.9025861157, 1.1654931969

C,0,4.7323875832,1.0181309009,0.6706755648

$\mathrm{H}, 0,5.316059095,0.1827841793,0.2700343131$

$\mathrm{H}, 0,5.2915080314,1.9409973033,0.4861269316$

H,0,4.6454628773,0.8893917043,1.7545373879

$\mathrm{E}(\mathrm{B} 3 \mathrm{LYP} / 6-31 \mathrm{G} *$ opt $)=$

$-352.920790$

Sum of electronic and zero-point Energies=

Sum of electronic and thermal Energies=

Sum of electronic and thermal Enthalpies=

Sum of electronic and thermal Free Energies=

$\mathrm{E}\left(\mathrm{B} 3 \mathrm{LYP} / 6-311+\mathrm{G}^{* *} / / \mathrm{B} 3 \mathrm{LYP} / 6-31 \mathrm{G}^{*}\right)=$

$-352.680272$

$-352.668345$

$-352.667400$

$-352.719524$

$\chi=-76.1$

$-353.012935$ 
$\%$ of total Charge on $\pi$ system (Mulliken analysis) $=54.6 \%$

9. (RR/SS)-Nona-3,5-dienyl cation (16b1, diethyl pentadienyl)

C, $0,-1.233470152,-0.0184180516,0.4571990983$

$\mathrm{H}, 0,-1.2334868585,-0.0180253737,1.5451402151$

C, $0,-0.0066330356,-0.0001403864,-0.2290401256$

$\mathrm{H}, 0,-0.0371755426,-0.0028220358,-1.3191312548$

C, $0,1.2567049464,0.0212729571,0.3873301107$

$\mathrm{H}, 0,1.3176493291,0.0261870037,1.473551096$

C, $0,-2.4242636082,-0.0321107742,-0.2275353072$

$\mathrm{H}, 0,-2.3863426475,-0.0367562574,-1.3189343125$

C, $0,2.407272772,0.031416227,-0.3630846369$

$\mathrm{H}, 0,2.3082745414,0.030776314,-1.4506571256$

C, $0,-3.7717851424,-0.0555696668,0.3814279888$

$\mathrm{H}, 0,-3.7087187231,-0.0338792278,1.4738667847$

C, $0,3.7868113264,0.057267739,0.1693048489$

$\mathrm{H}, 0,4.2858962112,-0.8642433027,-0.1733961296$

C, $0,4.5955948993,1.2645303605,-0.371006969$

$\mathrm{H}, 0,4.1609825169,2.2108799634,-0.0370071563$

$\mathrm{H}, 0,4.6333149285,1.2637845801,-1.4649803698$

C, $0,-4.6077146894,-1.2674255779,-0.1047979681$

$\mathrm{H}, 0,-4.7065495787,-1.2740835048,-1.1949272738$

$\mathrm{H}, 0,-4.1536539807,-2.2109918474,0.210677526$

$\mathrm{H}, 0,-4.2906968517,0.8629862994,0.061077753$

$\mathrm{H}, 0,3.7849749573,0.0427165568,1.2636874456$

$\mathrm{H}, 0,5.6201427744,1.2048917688,0.0048535142$

$\mathrm{H}, 0,-5.6097149753,-1.2063578959,0.3273970492$

$\mathrm{E}\left(\mathrm{B} 3 \mathrm{LYP} / 6-31 \mathrm{G}^{*}\right.$ opt $)=$

$-351.716340$

Sum of electronic and zero-point Energies $=\quad-351.499050$

Sum of electronic and thermal Energies $=\quad-351.487679$

Sum of electronic and thermal Enthalpies $=\quad-351.486735$

Sum of electronic and thermal Free Energies $=\quad-351.537117$

$\mathrm{E}\left(\mathrm{B} 3 \mathrm{LYP} / 6-311+\mathrm{G}^{* *} / / \mathrm{B} 3 \mathrm{LYP} / 6-31 \mathrm{G}^{*}\right)=\quad-351.806933$

$\chi=-68.6$

$\%$ of total Charge on $\pi$ system (Mulliken analysis) $=67.5 \%$

10. (RR/SS)-Deca-3,5-dienyl cation (16b2, ethyl propyl pentadienyl)

C, $0,-1.8699770187,-0.1412814826,0.4256064003$

$\mathrm{H}, 0,-1.8702403479,-0.1194891296,1.5133873203$

C, $0,-0.6419945076,-0.1481054148,-0.2604069651$

$\mathrm{H}, 0,-0.6730850711,-0.1729960345,-1.350225906$

C, $0,0.6208439692,-0.1280913937,0.3541021027$

$\mathrm{H}, 0,0.6834162861,-0.1022376799,1.4399674459$

C, $0,-3.0602494986,-0.1571202457,-0.2586380209$

$\mathrm{H}, 0,-3.0226961415,-0.1839837825,-1.3497531944$

C, $0,1.7720506793,-0.1512172368,-0.3974268335$

$\mathrm{H}, 0,1.6700568885,-0.1731271626,-1.4844424789$

C, $0,-4.4082509133,-0.1549343078,0.3508019024$

$\mathrm{H}, 0,-4.3440744306,-0.1221141788,1.44297483$

C, $0,3.148691968,-0.1186437698,0.1318088793$

$\mathrm{H}, 0,3.6840036055,-1.0004860651,-0.2549104574$

C, $0,3.9242907629,1.1458749467,-0.3559847329$

H,0,3.4007109386,2.0413452972,-0.0018254207 
$\mathrm{H}, 0,3.9076666608,1.177272696,-1.4522810954$

C, $0,5.3690803467,1.1422797482,0.150416741$

$\mathrm{H}, 0,5.9196188215,0.2701857179,-0.2189430809$

$\mathrm{H}, 0,5.890117864,2.0405242305,-0.1954146969$

$\mathrm{H}, 0,5.4100586669,1.1326213418,1.245032217$

C, $0,-5.2625471923,-1.3590207569,-0.1211373988$

H,0,-5.3666442954,-1.3734031991,-1.2107009647

$\mathrm{H}, 0,-4.8199993633,-2.3061934164,0.1999407195$

$\mathrm{H}, 0,-4.913876729,0.7680348209,0.0219013329$

$\mathrm{H}, 0,3.1550991486,-0.1732623452,1.2253400241$

H,0,-6.2617640759,-1.2811316307,0.3151009587

$\mathrm{E}\left(\mathrm{B} 3 \mathrm{LYP} / 6-31 \mathrm{G}^{*}\right.$ opt $)=$

$-391.031576$

Sum of electronic and zero-point Energies=

$-390.785662$

Sum of electronic and thermal Energies=

$-390.772947$

Sum of electronic and thermal Enthalpies=

$-390.772003$

Sum of electronic and thermal Free Energies=

$-390.826008$

$\mathrm{E}\left(\mathrm{B} 3 \mathrm{LYP} / 6-311+\mathrm{G}^{* *} / / \mathrm{B} 3 \mathrm{LYP} / 6-31 \mathrm{G}^{*}\right)=$

$-391.132713$

$\chi=-78.6$

$\%$ of total Charge on $\pi$ system (Mulliken analysis) $=66.5 \%$

11. (RR/SS)-Undeca-4,6-dienyl cation (16b3, dipropyl pentadienyl)

C, $0,-1.2242635011,0.2557536144,0.3121846419$

$\mathrm{H}, 0,-1.2549702775,0.2789211793,1.399509499$

C, $0,-0.0014725379,0.0053541201,-0.3337684668$

$\mathrm{H}, 0,-0.0004827678,-0.0125464629,-1.4241654798$

C, $0,1.2200056549,-0.2246241233,0.3223394715$

$\mathrm{H}, 0,1.2488218595,-0.2116009231,1.4098694815$

C, $0,-2.3726345038,0.478150181,-0.4091943789$

$\mathrm{H}, 0,-2.3045854019,0.4433577314,-1.4984833275$

C, $0,2.3686515583,-0.4748944603,-0.389277466$

$\mathrm{H}, 0,2.3025088088,-0.4755744895,-1.4792540444$

C, $0,-3.7117034118,0.7302156098,0.1581088339$

$\mathrm{H}, 0,-3.6687622402,0.8225251831,1.2483368055$

C, $0,3.7052853927,-0.7183255753,0.187565518$

$\mathrm{H}, 0,4.0624388191,-1.6931296967,-0.1816786375$

C, $0,4.7383906635,0.3582616992,-0.269046475$

$\mathrm{H}, 0,4.3966734283,1.3436148358,0.0683171038$

$\mathrm{H}, 0,4.7671949792,0.3872998153,-1.3652154992$

C, $0,6.1337011557,0.0631394018,0.28798231$

$\mathrm{H}, 0,6.5080577062,-0.904039464,-0.064998394$

$\mathrm{H}, 0,6.8387726261,0.8353065583,-0.0352728028$

$\mathrm{H}, 0,6.132611514,0.048685863,1.3833387552$

C, $0,-4.7296464125,-0.3787882174,-0.2547544796$

$\mathrm{H}, 0,-4.760310905,-0.4499758524,-1.3488946264$

$\mathrm{H}, 0,-4.3719401256,-1.3452000877,0.1189562215$

C, $0,-6.1280976039,-0.0841187899,0.2945285754$

$\mathrm{H}, 0,-6.5188362465,0.862630955,-0.094345851$

$\mathrm{H}, 0,-6.8214257474,-0.8793255704,0.0032652844$

$\mathrm{H}, 0,-6.1246845697,-0.0273168933,1.3884928847$

$\mathrm{H}, 0,-4.0823725762,1.6845954762,-0.2482730395$

$\mathrm{H}, 0,3.6619923915,-0.768968918,1.2804871605$

$\mathrm{E}(\mathrm{B} 3 \mathrm{LYP} / 6-31 \mathrm{G} *$ opt $)=$

$-430.346804$

Sum of electronic and zero-point Energies=

$-430.072183$ 


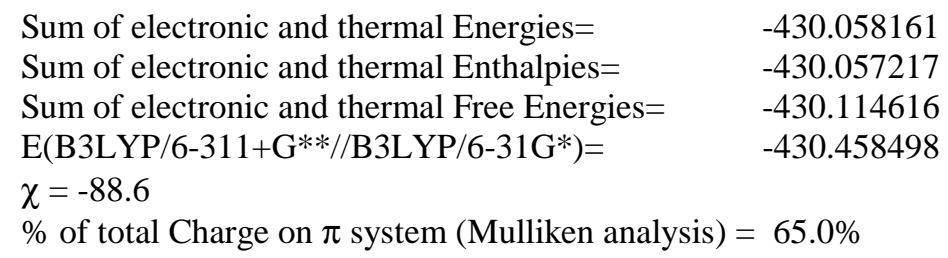

12. (RR/SS)-Undeca-3,5,7-trienyl cation (16c1, diethyl heptatrienyl)

C, $0,-1.2043673302,0.1495205842,0.2014888328$

$\mathrm{H}, 0,-1.1778074027,0.1531785678,1.2916385591$

C, $0,-0.0000075601,-0.0032908942,-0.4786428225$

$\mathrm{H}, 0,-0.0001969322,-0.0100825241,-1.5666015231$

C, $0,1.2045879506,-0.1475750366,0.2029255768$

$\mathrm{H}, 0,1.1784078689,-0.1376244915,1.2930451612$

C, $0,-2.4667528831,0.3023165855,-0.4182793312$

$\mathrm{H}, 0,-2.5195974353,0.3005836666,-1.505322582$

C, $0,2.4667677203,-0.3080547795,-0.4153268119$

$\mathrm{H}, 0,2.519220784,-0.319953886,-1.5023260452$

C, $0,-3.6091777647,0.4550885834,0.3170895571$

$\mathrm{H}, 0,-3.5233330047,0.4475001871,1.4056430547$

C, $0,3.6094677329,-0.4514922261,0.3214877182$

$\mathrm{H}, 0,3.5239970671,-0.4302792451,1.4098895175$

C, $0,4.978274298,-0.6225512772,-0.2260304962$

H, $0,4.9576081447,-0.6428136786,-1.3206083272$

$\mathrm{H}, 0,5.3513039064,-1.6026208174,0.1119483183$

C, $0,5.9522908338,0.4652644645,0.2859955773$

$\mathrm{H}, 0,6.0061722332,0.4706264005,1.3795871924$

$\mathrm{H}, 0,6.9551566698,0.2667342784,-0.1016188689$

H, $0,5.6450822247,1.4598482097,-0.0504746227$

C,0,-4.9781411336,0.6196765514,-0.2319827185

$\mathrm{H}, 0,-5.3501700086,1.6045347502,0.0930023572$

$\mathrm{H}, 0,-4.9580188857,0.6253010679,-1.3267457521$

C, $0,-5.9528263183,-0.4602887762,0.2951058302$

$\mathrm{H}, 0,-6.0062665824,-0.450791322,1.3886921254$

$\mathrm{H}, 0,-6.9556924777,-0.2662321008,-0.0947601567$

$\mathrm{H}, 0,-5.6465594431,-1.4595917386,-0.0279738811$

$\begin{array}{ll}\text { E(B3LYP/6-31G* opt })= & -429.137850 \\ \text { Sum of electronic and zero-point Energies= } & -428.886523 \\ \text { Sum of electronic and thermal Energies= } & -428.873046 \\ \text { Sum of electronic and thermal Enthalpies= } & -428.872102 \\ \text { Sum of electronic and thermal Free Energies= } & -428.927911 \\ \text { E(B3LYP/6-311+G**//B3LYP/6-31G*)= } & -429.248139 \\ \begin{array}{l}\chi=-80.8 \\ \% \text { of total Charge on } \pi \text { system (Mulliken analysis) }\end{array} & =73.5 \%\end{array}$

13. (RR/SS)-Dodeca-3,5,7-trienyl cation (16c2, ethyl propyl heptatrienyl)

C, $0,-0.5687860444,0.1094279154,0.2286591798$

$\mathrm{H}, 0,-0.5684475719,0.118816246,1.3191155501$

C, $0,0.661507285,0.1053516977,-0.422970096$

$\mathrm{H}, 0,0.6870001542,0.0955722346,-1.5106379177$

C, $0,1.8583704096,0.1144738114,0.2855643435$

$\mathrm{H}, 0,1.8069692974,0.1250621969,1.3747951651$

C, $0,-1.8244451399,0.1045478499,-0.4206883043$ 
$\mathrm{H}, 0,-1.8510124917,0.0943260708,-1.5086603218$

C, $0,3.1445795225,0.1106769515,-0.3045307076$

$\mathrm{H}, 0,3.2213655809,0.1001928353,-1.3901261764$

C, $0,-2.9952638442,0.120484306,0.2869625931$

$\mathrm{H}, 0,-2.9337361024,0.1268667707,1.3770912683$

C, $0,4.2800868317,0.1167282186,0.4561593071$

$\mathrm{H}, 0,4.1700718506,0.1327850254,1.5424309958$

C, $0,5.6708409326,0.1185858035,-0.0630034329$

H,0,5.6748211262,0.0865479324,-1.1575093329

H, $0,6.160516382,-0.8020032331,0.2925853905$

C, $0,6.4859308109,1.3276988448,0.454259087$

$\mathrm{H}, 0,6.5193953223,1.3484193791,1.5484814538$

$\mathrm{H}, 0,6.0579873915,2.2712998605,0.1031030226$

C, $0,-4.3577417105,0.104061282,-0.2945844795$

$\mathrm{H}, 0,-4.8910650472,1.0013242461,0.0577501963$

$\mathrm{H}, 0,-4.316150615,0.1538574119,-1.3882317231$

C, $0,-5.1738585254,-1.1392309266,0.1607534824$

$\mathrm{H}, 0,-5.2027809537,-1.1692494501,1.2572989952$

$\mathrm{H}, 0,-4.6530322987,-2.0465910948,-0.1675253042$

C, $0,-6.5980867435,-1.1173158185,-0.4009921783$

$\mathrm{H}, 0,-7.1493858861,-2.0046894501,-0.0744997105$

$\mathrm{H}, 0,-7.1487013861,-0.2343369605,-0.0576063087$

$\mathrm{H}, 0,-6.5954532658,-1.1097520171,-1.4966485601$

$\mathrm{H}, 0,7.5128358066,1.2586123829,0.0852605512$

$\mathrm{E}\left(\mathrm{B} 3 \mathrm{LYP} / 6-31 \mathrm{G}^{*}\right.$ opt $)=$

$-468.452694$

Sum of electronic and zero-point Energies=

Sum of electronic and thermal Energies=

$-468.172841$

$-468.157982$

$-468.157038$

Sum of electronic and thermal Enthalpies=

$-468.216718$

Sum of electronic and thermal Free Energies=

$-468.573538$

$\chi=-90.8$

$\%$ of total Charge on $\pi$ system (Mulliken analysis) $=72.8 \%$

14. (RR/SS)-Trideca-4,6,8-trienyl_cation (16c3, dipropyl heptatrienyl)

C, $0,-1.2201551573,0.0153327597,0.2444164295$

$\mathrm{H}, 0,-1.2206821989,0.0149422525,1.3349126318$

C, $0,0.0099632697,-0.0003425103,-0.4060046821$

$\mathrm{H}, 0,0.0364545477,-0.0004918166,-1.4936935809$

C, $0,1.2069601525,-0.0157942825,0.3035346902$

$\mathrm{H}, 0,1.1544006618,-0.0151121287,1.3927635209$

C, $0,-2.4757380726,0.0341886991,-0.4058677003$

$\mathrm{H}, 0,-2.5011612379,0.0346176091,-1.4939307068$

C, $0,2.4927094419,-0.0347301984,-0.2848534635$

$\mathrm{H}, 0,2.571062981,-0.0354712557,-1.3703893247$

C, $0,-3.6468181121,0.0591829201,0.3003295432$

$\mathrm{H}, 0,-3.5867472341,0.0540066074,1.3905367227$

C, $0,3.6280348718,-0.059354664,0.4775104417$

$\mathrm{H}, 0,3.5149795523,-0.0538749276,1.5635002305$

C,0,5.0168391662,-0.0674563472,-0.0387381391

$\mathrm{H}, 0,5.0268343601,-0.1309058841,-1.1324337197$

$\mathrm{H}, 0,5.5215883258,-0.9662427983,0.3497569081$

$\mathrm{C}, 0,5.8265889847,1.171909112,0.4383776116$

$\mathrm{H}, 0,5.8020648228,1.2181417146,1.5344762041$

$\mathrm{H}, 0,5.3346159316,2.0807672955,0.0718375797$ 
C, $0,-5.0088577777,0.0673979819,-0.2828857127$

$\mathrm{H}, 0,-5.5315764703,0.9665823906,0.0801014301$

$\mathrm{H}, 0,-4.9656068433,0.1302564574,-1.3758060029$

C, $0,-5.8413202298,-1.1714049035,0.1549528567$

$\mathrm{H}, 0,-5.8701368148,-1.2170346565,1.2509725735$

$\mathrm{H}, 0,-5.3324650524,-2.0806473574,-0.1867426002$

C, $0,7.2760897045,1.122655334,-0.0524064517$

H, 0,7.823209982,2.0078445879, 0.2867203366

$\mathrm{H}, 0,7.7969538464,0.238190188,0.3310736169$

$\mathrm{H}, 0,7.3268825956,1.0974399059,-1.146593466$

C,0,-7.2652294547,-1.12191015,-0.4057457709

$\mathrm{H}, 0,-7.8285409326,-2.0066942462,-0.0931047729$

$\mathrm{H}, 0,-7.8037669899,-0.2370243057,-0.048551717$

H,0,-7.2627645546,-1.0973321373,-1.5011237786

$\mathrm{E}\left(\mathrm{B} 3 \mathrm{LYP} / 6-31 \mathrm{G}^{*}\right.$ opt $)=$

$-507.767570$

Sum of electronic and zero-point Energies=

$-507.458917$

Sum of electronic and thermal Energies=

$-507.442779$

Sum of electronic and thermal Enthalpies=

$-507.441835$

Sum of electronic and thermal Free Energies=

$-507.504561$

$\mathrm{E}\left(\mathrm{B} 3 \mathrm{LYP} / 6-311+\mathrm{G} * * / / \mathrm{B} 3 \mathrm{LYP} / 6-31 \mathrm{G}^{*}\right)=$

$-507.899006$

$\chi=-101.0$

$\%$ of total Charge on $\pi$ system (Mulliken analysis) $=71.4 \%$

15. (RR/SS)-Trideca-3,5,7,9-tetraenyl cation (16d1, diethyl nonatetraenyl)

C,0,-3.6895907208,-0.0153505356,0.6358843777

$\mathrm{H}, 0,-3.6897998579,-0.0176975025,1.7245478733$

C, $0,-2.4405479431,-0.0103919129,-0.0396096735$

$\mathrm{H}, 0,-2.4633902507,-0.0092161351,-1.1298508798$

C, $0,-1.2049926223,-0.0058516661,0.5852995141$

$\mathrm{H}, 0,-1.1587002975,-0.0065454567,1.6725747353$

C, $0,-4.8735849249,-0.0131083849,-0.0397839068$

$\mathrm{H}, 0,-4.8427053116,-0.0156711457,-1.1312724297$

C, $0,-0.0093165705,0.0000846779,-0.1431776254$

C, $0,-6.2262439669,-0.0212865003,0.5789212466$

C, $0,1.2706826295,0.0052541258,0.4242117387$

C,0,-7.077871911,-1.2226737192,0.1099905531

$\mathrm{H}, 0,-7.1901247513,-1.2312250072,-0.9793758579$

$\mathrm{H}, 0,-6.6255745327,-2.1706512354,0.4167736949$

$\mathrm{H}, 0,-6.740264201,0.9047383346,0.276914249$

$\mathrm{H}, 0,-0.080127087,0.0007286421,-1.2313836576$

$\mathrm{H}, 0,1.3656839669,0.004663104,1.5083174825$

C,0,2.4148353699,0.0106253945,-0.3555412701

$\mathrm{H}, 0,2.2961973497,0.0107363089,-1.4395495973$

C,0,3.7408849648,0.01488366,0.1523947024

$\mathrm{H}, 0,3.882175708,0.0159477421,1.2318526931$

C,0,4.8273328188,0.013528424,-0.6710153971

$\mathrm{H}, 0,4.6552645108,0.017375147,-1.7492941272$

C, $0,6.2487657359,0.0210813041,-0.2328136983$

$\mathrm{H}, 0,6.7192431788,-0.9045463554,-0.5999804177$

C,0,7.0325329605,1.223087485,-0.8067369191

$\mathrm{H}, 0,6.6238783632,2.1706670714,-0.4428076526$

$\mathrm{H}, 0,7.0026655532,1.2329328559,-1.9014533082$

H,0,-6.1487307108,-0.0052087328,1.6713710237

H,0,6.3134789971,0.0037083003,0.8604491437 
$\mathrm{H}, 0,8.0797829141,1.1608066679,-0.4979438971$

$\mathrm{H}, 0,-8.0762684605,-1.1608367169,0.5519690758$

$\mathrm{E}\left(\mathrm{B} 3 \mathrm{LYP} / 6-31 \mathrm{G}^{*}\right.$ opt $)=$

Sum of electronic and zero-point Energies=

Sum of electronic and thermal Energies=

Sum of electronic and thermal Enthalpies=

Sum of electronic and thermal Free Energies=

$\mathrm{E}\left(\mathrm{B} 3 \mathrm{LYP} / 6-311+\mathrm{G}^{* *} / / \mathrm{B} 3 \mathrm{LYP} / 6-31 \mathrm{G}^{*}\right)=$

$\chi=-92.8$

$\%$ of total Charge on $\pi$ system (Mulliken analysis) $=78.3 \%$
$-506.555831$

$-506.270622$

$-506.255013$

$-506.254069$

$-506.315357$

$-506.685978$

16. (RR/SS)-Tetradeca-3,5,7,9-tetraenyl cation (16d2, ethyl propyl nonatetraenyl)

C, $0,-4.3490419168,-0.1028915924,0.6574369761$

$\mathrm{H}, 0,-4.3476828162,-0.098381247,1.7461200516$

C, $0,-3.1004408224,-0.1049144767,-0.0196141991$

$\mathrm{H}, 0,-3.1245517273,-0.1113088136,-1.1098185756$

C, $0,-1.8646245963,-0.0982173317,0.6038586051$

$\mathrm{H}, 0,-1.8173539563,-0.0912515196,1.6910944976$

C, $0,-5.5339110892,-0.1019074885,-0.0161749932$

$\mathrm{H}, 0,-5.5049477246,-0.1107822082,-1.1076829149$

C, $0,-0.6688905453,-0.0991196291,-0.1254205522$

C, $0,-6.8855737549,-0.1042144318,0.605266601$

C, $0,0.6107475923,-0.0911117303,0.4413323985$

C, $0,-7.7381738974,-1.3096745722,0.1488761297$

H, $0,-7.8557856785,-1.3258600544,-0.9398430295$

$\mathrm{H}, 0,-7.2834276274,-2.2551316741,0.4598094499$

$\mathrm{H}, 0,-7.4005031774,0.8190418183,0.2967017748$

$\mathrm{H}, 0,-0.7403025492,-0.1063526145,-1.2135744975$

$\mathrm{H}, 0,0.7060124539,-0.0838582381,1.5254078473$

C, $0,1.755575762,-0.0921378318,-0.3384673826$

$\mathrm{H}, 0,1.6369764767,-0.1001977687,-1.4224723804$

C, $0,3.0806694657,-0.0855707441,0.1695970301$

$\mathrm{H}, 0,3.2216960687,-0.0761275306,1.2490422648$

C,0,4.1684115067,-0.0974769559,-0.6533175896

$\mathrm{H}, 0,3.9957266153,-0.1030790096,-1.7314141205$

C, $0,5.5872176125,-0.0813252109,-0.2146335132$

$\mathrm{H}, 0,6.0791596958,-0.9853501023,-0.6074215017$

C, $0,6.3586059478,1.1525671922,-0.755061328$

$\mathrm{H}, 0,5.8796283407,2.0650019233,-0.3795533209$

H, $0,6.273914573,1.1796779929,-1.8490937584$

C, $0,7.8336991816,1.1264112907,-0.3456733102$

$\mathrm{H}, 0,8.3404319254,0.2377757888,-0.7389748423$

$\mathrm{H}, 0,8.3547710231,2.0081500047,-0.7319239542$

$\mathrm{H}, 0,7.9454644395,1.1234947658,0.7444805777$

$\mathrm{H}, 0,-6.8055342652,-0.078882826,1.6973964386$

$\mathrm{H}, 0,5.6552114312,-0.1231633278,0.878460886$

H,0,-8.7345261994,-1.245914284,0.5952298733

$\mathrm{E}\left(\mathrm{B} 3 \mathrm{LYP} / 6-31 \mathrm{G}^{*}\right.$ opt $)=$

$-545.870444$

Sum of electronic and zero-point Energies=

Sum of electronic and thermal Energies=

Sum of electronic and thermal Enthalpies=

Sum of electronic and thermal Free Energies=

$\mathrm{E}\left(\mathrm{B} 3 \mathrm{LYP} / 6-311+\mathrm{G}^{* *} / / \mathrm{B} 3 \mathrm{LYP} / 6-31 \mathrm{G}^{*}\right)=$

$-545.556710$

$-545.539722$

$-545.538777$

$-545.603769$

$-546.011150$ 
$\chi=-103.0$

$\%$ of total Charge on $\pi$ system (Mulliken analysis) $=76.5 \%$

17. (RR/SS)-Pentadeca-4,6,8,10-tetraenyl cation (16d3, dipropyl nonatetraenyl)

C, $0,-3.6965870761,0.4510930634,0.3398906392$

$\mathrm{H}, 0,-3.7664994677,0.4876992704,1.4257051331$

C, $0,-2.41633557,0.2808009088,-0.2491768617$

$\mathrm{H}, 0,-2.369159871,0.2468635666,-1.3381291376$

C, $0,-1.2316624507,0.1581605914,0.4572932598$

$\mathrm{H}, 0,-1.2554838806,0.1893418545,1.5448687191$

C, $0,-4.8261439483,0.5756503919,-0.4140438835$

$\mathrm{H}, 0,-4.7249498862,0.5307964243,-1.5002806165$

C, $0,-0.0000547211,-0.0048420099,-0.1881398115$

C, $0,-6.2048185735,0.7477271594,0.1113019475$

C, $0,1.2318080743,-0.1349127321,0.4642521454$

C, $0,-7.1541169592,-0.396657374,-0.3341008095$

H, $,-7.1431483053,-0.4684899021,-1.4293299369$

$\mathrm{H}, 0,-6.7674202004,-1.3493761016,0.0476835428$

C, $0,-8.5860793118,-0.1743167944,0.1602107668$

$\mathrm{H}, 0,-9.0044039118,0.7572197807,-0.2377028683$

$\mathrm{H}, 0,-9.2343771708,-0.996407808,-0.1592453431$

$\mathrm{H}, 0,-8.6274440131,-0.1222409779,1.2540873005$

$\mathrm{H}, 0,-6.6077992654,1.6937851639,-0.2837093855$

$\mathrm{H}, 0,-0.000264737,-0.0324952169,-1.2783108644$

$\mathrm{H}, 0,1.2560498365,-0.1109011023,1.5520003038$

C, $0,2.416211423,-0.2932083839,-0.2355444266$

$\mathrm{H}, 0,2.3686211064,-0.3145461844,-1.3247985219$

C, $0,3.6967030328,-0.4333186708,0.3609153628$

$\mathrm{H}, 0,3.7670275558,-0.4147635246,1.4471621553$

C, $0,4.8259936166,-0.5958704697,-0.3861469743$

$\mathrm{H}, 0,4.7244072086,-0.606207827,-1.4732225754$

C,0,6.2048710868,-0.7411291141,0.1467422394

$\mathrm{H}, 0,6.6076599759,-1.7059229958,-0.2002592432$

C,0,7.1540520664,0.3792616365,-0.3561332602

$\mathrm{H}, 0,6.7674512224,1.3500557318,-0.0228674525$

$\mathrm{H}, 0,7.1427782056,0.3956746854,-1.4535923817$

C, $0,8.5861499631,0.1822010206,0.1483907067$

$\mathrm{H}, 0,9.0043503373,-0.7683251251,-0.2018997442$

$\mathrm{H}, 0,9.2343766887,0.9870144041,-0.212514961$

$\mathrm{H}, 0,8.6278336342,0.1856510715,1.2434893545$

$\mathrm{H}, 0,-6.1954751644,0.829510489,1.2042133571$

H,0,6.1959261889,-0.7677710153,1.2423869239

$\mathrm{E}\left(\mathrm{B} 3 \mathrm{LYP} / 6-31 \mathrm{G}^{*} \mathrm{opt}\right)=$

$-585.185100$

Sum of electronic and zero-point Energies $=\quad-584.842590$

Sum of electronic and thermal Energies $=\quad-584.824312$

Sum of electronic and thermal Enthalpies $=\quad-584.823368$

Sum of electronic and thermal Free Energies $=\quad-584.891570$

$\mathrm{E}\left(\mathrm{B} 3 \mathrm{LYP} / 6-311+\mathrm{G}^{* *} / / \mathrm{B} 3 \mathrm{LYP} / 6-31 \mathrm{G}^{*}\right)=\quad-585.336410$

$\chi=-113.1$

$\%$ of total Charge on $\pi$ system (Mulliken analysis) $=75.6 \%$

18. (RR/SS)-Pentadeca-3,5,7,9,11-pentaenyl cation (16e1, diethyl undecapentaenyl)

C,0,-3.6430226401,0.2954752824,0.2079808451 
$\mathrm{H}, 0,-3.5772192383,0.2988730379,1.2964945051$

C, $0,-2.4710647698,0.1949818981,-0.5140912524$

$\mathrm{H}, 0,-2.5155727376,0.190390857,-1.6016857455$

C,0,-1.2151547565,0.0983915056,0.1094961215

$\mathrm{H}, 0,-1.1928302933,0.1047027584,1.1997860792$

C, $0,-4.9429819335,0.3970269834,-0.3637907039$

$\mathrm{H}, 0,-5.0290671553,0.3942574215,-1.4493246391$

C, $0,-0.000038219,-0.0043443466,-0.5638895617$

C, $0,-6.064469806,0.4997009456,0.3995841902$

C, $0,1.2151473045,-0.0973608501,0.1107835082$

C, $0,-7.4587046233,0.6050758332,-0.1144806653$

$\mathrm{H}, 0,-7.8721051499,1.5698070822,0.2183021286$

$\mathrm{H}, 0,-7.4616993758,0.6157897856,-1.2099931596$

C, $0,-8.3681205301,-0.5232263911,0.4191232425$

$\mathrm{H}, 0,-8.3995350964,-0.5257638561,1.5140074021$

$\mathrm{H}, 0,-9.3890378783,-0.3816348111,0.0528963404$

$\mathrm{H}, 0,-8.0173846717,-1.504337592,0.0842401131$

$\mathrm{H}, 0,-5.9505861238,0.4953544912,1.4855470125$

H, $,-0.0000914354,-0.0121613772,-1.6523343082$

$\mathrm{H}, 0,1.192929813,-0.0880082726,1.2010537818$

C, $0,2.471002888,-0.2028110227,-0.5114756607$

$\mathrm{H}, 0,2.5154032093,-0.2138466783,-1.5990282729$

C, $0,3.6430465319,-0.2927574593,0.211848561$

$\mathrm{H}, 0,3.577353786,-0.2805082936,1.3003052611$

C,0,4.9429656996,-0.4022954889,-0.3585336089

H, 0,5.0289324725,-0.4151645901,-1.4440056406

C, $0,6.0645528671,-0.4936743329,0.4061345083$

$\mathrm{H}, 0,5.9507390728,-0.4736856734,1.4919262071$

C,0,7.4587711413,-0.6060515402,-0.1065601025

$\mathrm{H}, 0,7.4615877115,-0.6320507417,-1.2018269306$

$\mathrm{H}, 0,7.8723732184,-1.5660042499,0.2395025024$

C, $0,8.3680789816,0.5297342422,0.4110552352$

$\mathrm{H}, 0,8.3996170838,0.547604088,1.5057927831$

$\mathrm{H}, 0,9.3889782841,0.3831830553,0.0467318491$

$\mathrm{H}, 0,8.0171656892,1.5060120068,0.0625047894$

$\mathrm{E}\left(\mathrm{B} 3 \mathrm{LYP} / 6-31 \mathrm{G}^{*} \mathrm{opt}\right)=$

Sum of electronic and zero-point Energies=

Sum of electronic and thermal Energies=

Sum of electronic and thermal Enthalpies=

Sum of electronic and thermal Free Energies=

$\mathrm{E}\left(\mathrm{B} 3 \mathrm{LYP} / 6-311+\mathrm{G}^{* *} / / \mathrm{B} 3 \mathrm{LYP} / 6-31 \mathrm{G}^{*}\right)=$

$\chi=-104.7$

$\%$ of total Charge on $\pi$ system (Mulliken analysis) $=81.4 \%$
$-583.971543$

$-583.652540$

$-583.634787$

$-583.633843$

$-583.700576$

$-584.121669$

19. (RR/SS)-Hexadeca-3,5,7,9,11-pentaenyl cation (16e2, ethyl propyl undecapentaenyl)

C, $0,-4.325644721,-0.0892689608,-0.0437062478$

$\mathrm{H}, 0,-4.3271747988,-0.0990633487,1.0467606899$

C, $0,-3.1076253398,-0.0832808283,-0.6922440903$

$\mathrm{H}, 0,-3.08490828,-0.072818918,-1.78048904$

C, $0,-1.8881314169,-0.0888508052,0.0072032615$

$\mathrm{H}, 0,-1.9331200898,-0.0996837028,1.0967662346$

C, $0,-5.5923654597,-0.0822287777,-0.6942427362$

$\mathrm{H}, 0,-5.6111943356,-0.0744636567,-1.7830172166$

C, $0,-0.6301969328,-0.0810155155,-0.5902807577$ 
C, $0,-6.7630523462,-0.0806595984,-0.0013987128$

C, $0,0.5456054343,-0.0863452951,0.1574486997$

C, $0,-8.127219707,-0.0765628117,-0.6003700425$

$\mathrm{H}, 0,-8.6316383929,0.8520681325,-0.2910587683$

$\mathrm{H}, 0,-8.0634830511,-0.0571191487,-1.6939589255$

C, $0,-8.9804871972,-1.2735932495,-0.1279354137$

$\mathrm{H}, 0,-9.0811661592,-1.2854349784,0.9627235038$

$\mathrm{H}, 0,-8.5358634687,-2.2233431215,-0.4410731985$

$\mathrm{H}, 0,-6.7160577936,-0.0930106305,1.0894550931$

$\mathrm{H}, 0,-0.563145206,-0.0699489803,-1.6766437451$

$\mathrm{H}, 0,0.4562625695,-0.0976926923,1.244271759$

C, $0,1.8409031605,-0.0782958462,-0.3867757019$

$\mathrm{H}, 0,1.9522034702,-0.0672775866,-1.4695375171$

C, $0,2.9708245561,-0.0843209545,0.4066955787$

$\mathrm{H}, 0,2.8382282887,-0.0957856574,1.4890731488$

C, $0,4.3068707804,-0.0791479406,-0.08320622$

$\mathrm{H}, 0,4.4595225052,-0.0675291686,-1.1613240565$

C,0,5.3843714179,-0.0946850431,0.7484252517

$\mathrm{H}, 0,5.2023062512,-0.1019240284,1.8249773204$

C, $0,6.8096988237,-0.0821765463,0.3223346175$

$\mathrm{H}, 0,6.8852405491,-0.1221091113,-0.7706237887$

$\mathrm{H}, 0,7.2958703621,-0.9895742083,0.7142658161$

C, $0,7.5815720661,1.1469329878,0.8674037487$

$\mathrm{H}, 0,7.485827792,1.177203259,1.9606212494$

$\mathrm{H}, 0,7.1109414591,2.0615172415,0.4861465548$

C, $0,9.0611494454,1.1146046895,0.474868662$

$\mathrm{H}, 0,9.583176771,1.9942393678,0.8648621675$

$\mathrm{H}, 0,9.5593696485,0.2240197647,0.8748922649$

$\mathrm{H}, 0,9.1850850288,1.1085551342,-0.6141128514$

H,0,-9.9839185026,-1.207456988,-0.5582960753

$\mathrm{E}\left(\mathrm{B} 3 \mathrm{LYP} / 6-31 \mathrm{G}^{*}\right.$ opt $)=$

$-623.286022$

Sum of electronic and zero-point Energies= $\quad-622.938426$

Sum of electronic and thermal Energies= $\quad-622.919319$

Sum of electronic and thermal Enthalpies $=\quad-622.918375$

Sum of electronic and thermal Free Energies= $\quad-622.988677$

$\mathrm{E}\left(\mathrm{B} 3 \mathrm{LYP} / 6-311+\mathrm{G}^{* *} / / \mathrm{B} 3 \mathrm{LYP} / 6-31 \mathrm{G}^{*}\right)=\quad-623.446725$

$\chi=-114.9$

$\%$ of total Charge on $\pi$ system (Mulliken analysis) $=80.3 \%$

20. (RR/SS)-Heptadeca-4,6,8,10,12-pentaenyl cation (16e3, dipropyl undecapentaenyl)

C, $0,-3.6625001414,0.0127412478,0.0123424343$

$\mathrm{H}, 0,-3.6619589396,0.0109592776,1.1028709721$

C, $0,-2.4450113768,0.0089978388,-0.6382599932$

$\mathrm{H}, 0,-2.4241038119,0.0108782934,-1.726586871$

C, $0,-1.2249874622,0.0038058712,0.0592213323$

$\mathrm{H}, 0,-1.2684415128,0.0026056793,1.1489073682$

C,0,-4.9297975913,0.0214524329,-0.6354653891

$\mathrm{H}, 0,-4.9510576799,0.0233026321,-1.7242038287$

C,0,0.0326747332,0.0004866986,-0.5398144237

C,0,-6.0995603217,0.0342178309,0.06015487

C, $0,1.2088924412,-0.0040178997,0.2065505467$

C,0,-7.4634406105,0.0362844082,-0.534385626

$\mathrm{H}, 0,-7.987169988,0.9439336016,-0.1948958095$

$\mathrm{H}, 0,-7.4066356746,0.0867381445,-1.6280456944$ 
C,0,-8.3033298515,-1.1915439417,-0.0982720345

$\mathrm{H}, 0,-8.33876657,-1.2330737004,0.9981961768$

$\mathrm{H}, 0,-7.7969974725,-2.1062522043,-0.4303057168$

$\mathrm{H}, 0,-6.0485286152,0.0278761493,1.1508162409$

$\mathrm{H}, 0,0.098446559,0.0014435882,-1.6263141653$

H, $0,1.1205814643,-0.0047350219,1.2935215686$

C, $0,2.504144237,-0.0081164158,-0.3386755209$

$\mathrm{H}, 0,2.6146732317,-0.0080844843,-1.4215787479$

C,0,3.6342614346,-0.0131333609,0.4540327131

$\mathrm{H}, 0,3.5021747284,-0.0132654699,1.5365339953$

C,0,4.9704499377,-0.0208453571,-0.0361861932

$\mathrm{H}, 0,5.1228883763,-0.0207854149,-1.1144112745$

C,0,6.0477597302,-0.0349616905,0.7954384553

H, $0,5.8655341323,-0.030529754,1.8719889568$

C, $0,7.4733999657,-0.0361397239,0.3697614068$

H, $0,7.5489420689,-0.0846389225,-0.7228529725$

$\mathrm{H}, 0,7.952431849,-0.9444602323,0.768328733$

C, $0,8.2544396083,1.1908009238,0.9061962511$

$\mathrm{H}, 0,8.1573471394,1.2303713443,1.9990047153$

$\mathrm{H}, 0,7.7917780171,2.1061649243,0.5171420906$

C, $0,9.7344162625,1.1437071265,0.5164483083$

$\mathrm{H}, 0,10.2628627841,2.0219969035,0.9007513667$

$\mathrm{H}, 0,10.2247646494,0.2522197064,0.9240431468$

$\mathrm{H}, 0,9.8601876731,1.1284347021,-0.5722307843$

C,0,-9.7254897247,-1.1436240525,-0.6636189561

$\mathrm{H}, 0,-10.2963227257,-2.0225530579,-0.3474370224$

$\mathrm{H}, 0,-10.2615401333,-0.2528235911,-0.3165607446$

H,0,-9.7190171702,-1.1263947135,-1.7594907879

$\mathrm{E}\left(\mathrm{B} 3 \mathrm{LYP} / 6-31 \mathrm{G}^{*}\right.$ opt $)=$

$-662.600516$

Sum of electronic and zero-point Energies $=\quad-662.224233$

Sum of electronic and thermal Energies $=\quad-662.203802$

Sum of electronic and thermal Enthalpies $=\quad-662.202858$

Sum of electronic and thermal Free Energies $=\quad-662.276560$

$\mathrm{E}\left(\mathrm{B} 3 \mathrm{LYP} / 6-311+\mathrm{G}^{* *} / / \mathrm{B} 3 \mathrm{LYP} / 6-31 \mathrm{G}^{*}\right)=\quad-662.771816$

$\chi=-125.1$

$\%$ of total Charge on $\pi$ system (Mulliken analysis) $=79.2 \%$

21. (RR/SS)-Hex-3-ene (17a1, diethyl ethene)

C, $0,-1.9465570246,0.1508826906,0.2621028639$

$\mathrm{H}, 0,-1.8709391914,0.1270953176,1.3567999273$

C, $0,-0.5696940361,0.076519402,-0.3414624329$

$\mathrm{H}, 0,-0.5249235655,0.1389437298,-1.4319012139$

C, $0,0.5690900732,-0.0752245055,0.3401396329$

$\mathrm{H}, 0,0.5243380072,-0.1374973432,1.4305941955$

C, $0,1.9459498008,-0.1498891513,-0.2634254626$

H, $0,2.4059357291,-1.1163193446,-0.0070734896$

C, $0,2.8725259916,0.9783873272,0.2216555395$

$\mathrm{H}, 0,2.9749890478,0.963798005,1.3135383459$

$\mathrm{H}, 0,2.4752825022,1.9595154858,-0.0613543245$

$\mathrm{H}, 0,-2.4080782324,1.1158446906,0.0029766533$

C, $0,-2.8715148066,-0.9802578384,-0.2194557204$

$\mathrm{H}, 0,-2.9751070249,-0.9683806308,-1.3112618964$

$\mathrm{H}, 0,-2.4720942338,-1.9599578325,0.065424529$

$\mathrm{H}, 0,1.8705367214,-0.1227756914,-1.358068652$ 
$\mathrm{H}, 0,-3.8741901811,-0.8811121015,0.2121568678$

$\mathrm{H}, 0,3.8754504318,0.8783381681,-0.2091574647$

$\mathrm{E}\left(\mathrm{B} 3 \mathrm{LYP} / 6-31 \mathrm{G}^{*}\right.$ opt $)=$

$-235.853957$

Sum of electronic and zero-point Energies=

Sum of electronic and thermal Energies=

Sum of electronic and thermal Enthalpies=

Sum of electronic and thermal Free Energies=

$\mathrm{E}\left(\mathrm{B} 3 \mathrm{LYP} / 6-311+\mathrm{G}^{* *} / / \mathrm{B} 3 \mathrm{LYP} / 6-31 \mathrm{G}^{*}\right)=$

$\chi=-57.9$

$-235.687769$

$-235.679784$

$-235.678839$

$-235.719946$

$-235.922747$

22. (RR/SS)-Hept-3-ene (17a2, ethyl propyl ethene)

C,0,-2.5980490336,-0.1100441576,0.1566502622

$\mathrm{H}, 0,-2.5956181498,-0.1259414207,1.254173473$

C, $0,-1.1815692622,-0.1155603093,-0.352208332$

$\mathrm{H}, 0,-1.0663071488,-0.0577298767,-1.437688336$

C, $0,-0.0850222245,-0.2031828977,0.4058373823$

$\mathrm{H}, 0,-0.2009286685,-0.2618581898,1.4911128765$

C, $0,1.3306425541,-0.2075923551,-0.1038618628$

$\mathrm{H}, 0,1.8302523061,-1.1397574387,0.2044969884$

C,0,2.1641519,0.9822904738,0.4100360052

$\mathrm{H}, 0,2.1423253768,0.9907385509,1.5088429036$

C,0,3.6169644914,0.9463555419,-0.0739603582

$\mathrm{H}, 0,3.6702591876,0.9642100044,-1.1695863864$

$\mathrm{H}, 0,4.1261537098,0.0360673273,0.2662905428$

$\mathrm{H}, 0,1.6860627356,1.9169109812,0.0882305189$

$\mathrm{H}, 0,-3.089494162,0.8291654606,-0.139739839$

C, $0,-3.4294167191,-1.2888607785,-0.3781660454$

$\mathrm{H}, 0,-3.4577317597,-1.2874586938,-1.4745691826$

$\mathrm{H}, 0,-3.0019209385,-2.2457764806,-0.0585430384$

$\mathrm{H}, 0,1.3293527998,-0.2058633595,-1.2024309066$

$\mathrm{H}, 0,4.1846366738,1.8056814851,0.3008241914$

H,0,-4.463252199,-1.2388214547,-0.0173761147

$\mathrm{E}\left(\mathrm{B} 3 \mathrm{LYP} / 6-31 \mathrm{G}^{*}\right.$ opt $)=$

$-275.167863$

Sum of electronic and zero-point Energies=

$-274.973092$

$-274.963760$

Sum of electronic and thermal Energies=

Sum of electronic and thermal Enthalpies=

$-274.962816$

Sum of electronic and thermal Free Energies=

$-275.007544$

$\mathrm{E}\left(\mathrm{B} 3 \mathrm{LYP} / 6-311+\mathrm{G}^{* *} / / \mathrm{B} 3 \mathrm{LYP} / 6-31 \mathrm{G}^{*}\right)=$

$-275.247237$

$\chi=-68.0$

23. (RR/SS)-Oct-4-ene (17a3, dipropyl ethene)

C, $0,-1.9351267182,0.1962928845,0.3162746674$

$\mathrm{H}, 0,-1.8316173759,0.1561080425,1.4091706348$

C, $0,-0.5778565237,0.105591892,-0.3267994052$

$\mathrm{H}, 0,-0.5597780945,0.2002284867,-1.4155876642$

C, $0,0.5741909671,-0.0974562769,0.3185629666$

$\mathrm{H}, 0,0.5559531353,-0.1918901037,1.407390363$

C, $0,1.9315743133,-0.1894720262,-0.3240260982$

H, $0,2.3777704344,-1.1721409894,-0.1026920638$

C,0,2.9049161536,0.9043447698,0.1563087506

$\mathrm{H}, 0,2.9839994829,0.8614176664,1.2516638691$

C,0,4.2995780828,0.7767729189,-0.4640589816 
$\mathrm{H}, 0,4.2545364225,0.8445102671,-1.5581010205$

$\mathrm{H}, 0,4.7594153295,-0.1868796842,-0.2118465586$

$\mathrm{H}, 0,2.4792569477,1.888411294,-0.0805026894$

$\mathrm{H}, 0,-2.3884088221,1.1728837176,0.0830339101$

C,0,-2.9013937846,-0.9104749911,-0.1486157416

$\mathrm{H}, 0,-2.9810618541,-0.8834728859,-1.2444400574$

$\mathrm{H}, 0,-2.4690490413,-1.8882288816,0.1017916138$

C, $0,-4.2967045656,-0.7835480588,0.4704463688$

$\mathrm{H}, 0,-4.7634377372,0.1730030142,0.2042748027$

$\mathrm{H}, 0,-4.9607116747,-1.5851772634,0.1274271734$

$\mathrm{H}, 0,-4.2508009837,-0.8348406464,1.5653549673$

$\mathrm{H}, 0,1.8298046868,-0.1348010041,-1.4164483044$

H,0,4.969061597, 1.5685622979,-0.1090441364

$\mathrm{E}\left(\mathrm{B} 3 \mathrm{LYP} / 6-31 \mathrm{G}^{*} \mathrm{opt}\right)=$

$-314.481741$

$-314.258489$

$-314.247768$

$-314.246824$

$-314.295411$

$-314.571693$

E(B3LYP
$\chi=-78.0$

24. (RR/SS)-Octa-3,5-diene (17b1, diethyl butadiene)

C, $0,-0.6449226313,0.3010862769,-0.1503293792$

$\mathrm{H}, 0,-0.6586317684,1.0533552924,-0.9414116013$

C, $0,0.6455964578,-0.3015350665,0.1476869165$

$\mathrm{H}, 0,0.6592714628,-1.053987152,0.9385985282$

C, $0,1.7943093959,0.0038993451,-0.4791419244$

$\mathrm{H}, 0,1.7757943217,0.7605918015,-1.2669811192$

C, $0,-1.7935740336,-0.0040944669,0.476730539$

$\mathrm{H}, 0,-1.7750243473,-0.7606361197,1.2647195375$

C, $0,3.1364242619,-0.6003583576,-0.1782244671$

H, $0,3.5186230799,-1.109417515,-1.0764585549$

C,0,-3.1355468592,0.6007561911,0.1763684219

C, $0,4.1753543812,0.4425100989,0.2714580029$

$\mathrm{H}, 0,3.8578448054,0.9377464538,1.1956648707$

C, $0,-4.1772282025,-0.4424550955,-0.2659439563$

$\mathrm{H}, 0,-3.8607420972,-0.9456518175,-1.1862040078$

$\mathrm{H}, 0,-4.3171897877,-1.2120673832,0.5027773915$

$\mathrm{H}, 0,4.3125477553,1.2183778733,-0.4914493851$

$\mathrm{H}, 0,-3.0283528974,1.3700007323,-0.5992140632$

H, $0,-3.5147113198,1.1144731567,1.0732694626$

$\mathrm{H}, 0,3.0284738405,-1.3731877605,0.5936796688$

$\mathrm{H}, 0,-5.1504067287,0.0271239635,-0.4497621095$

H,0,5.1500270597,-0.0255750786,0.4511464617

$\mathrm{E}\left(\mathrm{B} 3 \mathrm{LYP} / 6-31 \mathrm{G}^{*} \mathrm{opt}\right)=$

$-313.259046$

Sum of electronic and zero-point Energies= $-313.059348$

Sum of electronic and thermal Energies=

$-313.049145$

Sum of electronic and thermal Enthalpies=

$-313.048201$

Sum of electronic and thermal Free Energies=

$-313.095304$

$\mathrm{E}\left(\mathrm{B} 3 \mathrm{LYP} / 6-311+\mathrm{G}^{* *} / / \mathrm{B} 3 \mathrm{LYP} / 6-31 \mathrm{G}^{*}\right)=$

$-313.348213$

$\chi=-69.8$

25. (RS/SR)-Octa-3,5-diene (iso-17b1, diethyl butadiene) 
C, $0,-0.6510708536,-0.3623189302,0.3248430794$

$\mathrm{H}, 0,-0.6495338657,-0.3613154998,1.4165637756$

C, $0,0.6512033839,-0.3624359859,-0.3244466231$

$\mathrm{H}, 0,0.6496661735,-0.3626291895,-1.4161677482$

C,0,1.827991314,-0.3646558411,0.3242910935

$\mathrm{H}, 0,1.8234769368,-0.3614615109,1.4167771332$

C, $0,-1.8278576945,-0.3656808486,-0.3238916159$

$\mathrm{H}, 0,-1.8233441883,-0.3636818022,-1.4163804863$

C, $0,3.1811572545,-0.3530149537,-0.3283320863$

H, $0,3.0655806471,-0.4012210444,-1.4187136435$

C, $0,-3.1810278763,-0.3538203686,0.3287187474$

C, $0,4.0185431659,0.8811202398,0.0522960374$

$\mathrm{H}, 0,4.1607557678,0.9426580541,1.1379820462$

C, $0,-4.0188652562,0.8795909373,-0.0532592329$

$\mathrm{H}, 0,-3.5253405925,1.8030935229,0.2689788188$

H,0,-4.1611012006, $0.9398885326,-1.1390117726$

$\mathrm{H}, 0,3.5246809115,1.8040888669,-0.2709530326$

$\mathrm{H}, 0,-3.0654337027,-0.4007909473,1.4191523938$

H, $0,-3.736744087,-1.2579401254,0.0356974806$

H, $0,3.7372040696,-1.2566100885,-0.0343214712$

$\mathrm{H}, 0,-5.0104141749,0.8409165678,0.4122200728$

H,0,5.0101066788,0.8422991698,-0.4131399636

$\mathrm{E}\left(\mathrm{B} 3 \mathrm{LYP} / 6-31 \mathrm{G}^{*}\right.$ opt $)=$

$-313.259067$

Sum of electronic and zero-point Energies $=\quad-313.059268$

Sum of electronic and thermal Energies= $\quad-313.049115$

Sum of electronic and thermal Enthalpies $=\quad-313.048171$

Sum of electronic and thermal Free Energies $=\quad-313.095086$

$\mathrm{E}\left(\mathrm{B} 3 \mathrm{LYP} / 6-311+\mathrm{G}^{* *} / / \mathrm{B} 3 \mathrm{LYP} / 6-31 \mathrm{G}^{*}\right)=\quad-313.348252$

$\chi=-69.9$

26. (RR/SS)-Nona-3,5-diene (17b2, ethyl propyl butadiene)

C, $0,-1.2911652602,-0.1402799677,0.4002283966$

H, $0,-1.2878499117,-0.164381334,1.4916780649$

C, $0,0.0101578216,-0.1343826653,-0.2508347763$

H, $0,0.0070179321,-0.1098077379,-1.3422480281$

C, $0,1.1878335224,-0.1603850914,0.396032177$

$\mathrm{H}, 0,1.1837562557,-0.1824562723,1.488243171$

C, $0,-2.4690890122,-0.1159047272,-0.2460615928$

H, $0,-2.4666019792,-0.0955620338,-1.3384042038$

C, $0,2.539776493,-0.1436304221,-0.2576879709$

$\mathrm{H}, 0,2.424957462,-0.1706958465,-1.3497942458$

C,0,-3.8211333305,-0.131961646,0.4088328457

C, $0,3.3913440284,1.0785651299,0.1403976137$

$\mathrm{H}, 0,3.4818881566,1.1132416133,1.2351307681$

C,0,4.7873976519,1.0632456401,-0.4895743078

$\mathrm{H}, 0,4.7287982425,1.0607384161,-1.5850051501$

$\mathrm{H}, 0,5.3493986621,0.1709679248,-0.1868884725$

C, $0,-4.6704472115,-1.3481534663,-0.0019638893$

$\mathrm{H}, 0,-4.8137683099,-1.380659226,-1.0887559707$

$\mathrm{H}, 0,-4.1849896935,-2.2835454591,0.297411976$

$\mathrm{H}, 0,2.8592664762,1.9935146242,-0.1511994728$

$\mathrm{H}, 0,-4.3694376476,0.7839526787,0.1393694133$

H,0,5.3698833968, 1.9417770493,-0.1896608263 
H,0,-3.7031167557,-0.1131269008,1.4998347686

$\mathrm{H}, 0,3.0941359102,-1.0549137143,0.019052608$

$\mathrm{H}, 0,-5.6613864139,-1.3117204854,0.4650246248$

$\mathrm{E}\left(\mathrm{B} 3 \mathrm{LYP} / 6-31 \mathrm{G}^{*}\right.$ opt $)=$

$-352.572986$

Sum of electronic and zero-point Energies=

Sum of electronic and thermal Energies=

Sum of electronic and thermal Enthalpies=

Sum of electronic and thermal Free Energies= $\mathrm{E}\left(\mathrm{B} 3 \mathrm{LYP} / 6-311+\mathrm{G}^{* *} / / \mathrm{B} 3 \mathrm{LYP} / 6-31 \mathrm{G}^{*}\right)=$

$-352.344579$

$-352.333066$

$-352.332122$

$-352.382552$

$\chi=-79.9$

$-352.672750$

27. (RR/SS)-Deca-4,6-diene (17b3, dipropyl butadiene)

C, $0,-0.6496741905,-0.0062769586,0.3272935306$

$\mathrm{H}, 0,-0.6441874455,-0.0458455807,1.4183259656$

C, $0,0.6501003351,0.0055254399,-0.3266426058$

$\mathrm{H}, 0,0.6446188093,0.045070934,-1.4176769374$

C, $0,1.8293478184,-0.0315820456,0.3168420942$

$\mathrm{H}, 0,1.8280414675,-0.0686493677,1.4086203071$

C, $0,-1.8289214365,0.0308417822,-0.3161883555$

$\mathrm{H}, 0,-1.8276185678,0.0678964233,-1.4079687727$

C, $0,3.1795793823,-0.0083982877,-0.3400847218$

$\mathrm{H}, 0,3.0623247048,-0.026835195,-1.4321724465$

C, $0,-3.1791601974,0.0078807112,0.34072754$

C,0,4.0309428883,1.2116145137,0.0654229735

$\mathrm{H}, 0,4.1245579458,1.2378495096,1.1601353464$

C, $0,5.4250903099,1.2028706533,-0.5687959755$

H, $0,5.3630304868,1.2072586948,-1.6640458977$

$\mathrm{H}, 0,5.9897854845,0.3097994187,-0.2735882385$

C,0,-4.0315403762,-1.2108788473,-0.0663709422

$\mathrm{H}, 0,-4.1253245548,-1.2355035362,-1.1611062308$

$\mathrm{H}, 0,-3.4981023119,-2.1281528381,0.2152359549$

C, $0,-5.4255950457,-1.2019273104,0.5680493928$

H, $0,-5.9895067184,-0.3078426598,0.2744140296$

$\mathrm{H}, 0,-6.0082185635,-2.0786062875,0.2630230188$

$\mathrm{H}, 0,-5.3633962149,-1.2082046463,1.6632820022$

$\mathrm{H}, 0,3.4968006656,2.1280590286,-0.2175406355$

$\mathrm{H}, 0,-3.73440793,0.9215783826,0.0740412855$

$\mathrm{H}, 0,6.006868847,2.080595125,-0.2651652164$

$\mathrm{H}, 0,-3.0618585567,0.024786388,1.4328346527$

H,0,3.73555755262,-0.9212716978,-0.0721657686

$\mathrm{E}\left(\mathrm{B} 3 \mathrm{LYP} / 6-31 \mathrm{G}^{*}\right.$ opt $)=$

$-391.886898$

Sum of electronic and zero-point Energies=

Sum of electronic and thermal Energies=

Sum of electronic and thermal Enthalpies=

Sum of electronic and thermal Free Energies=

$\mathrm{E}\left(\mathrm{B} 3 \mathrm{LYP} / 6-311+\mathrm{G}^{* *} / / \mathrm{B} 3 \mathrm{LYP} / 6-31 \mathrm{G}^{*}\right)=$

$-391.629934$

$-391.617065$

$-391.616120$

$-391.670203$

$\chi=-90.0$

$-391.997256$

28. (RR/SS)-Deca-3,5,7-triene (17c1, diethyl hexatriene)

C, $0,-0.5846392172,0.239626722,0.2422716379$

$\mathrm{H}, 0,-0.5706533866,1.1508398107,0.8424579096$

C, $, 0,0.5846416265,-0.2396333921,-0.24228235$ 
$\mathrm{H}, 0,0.5706562748,-1.1508468192,-0.8424680612$ C, $0,1.8777505696,0.3746349467,-0.0221714915$ $\mathrm{H}, 0,1.8879450163,1.2864845234,0.5779884413$ C, $0,-1.8777489207,-0.3746397771,0.0221601239$

$\mathrm{H}, 0,-1.8879455848,-1.2864858361,-0.5780051131$ C,0,3.042270019,-0.0986457004,-0.5040050561 $\mathrm{H}, 0,3.0279174889,-1.0134911981,-1.1007174931$ C, $0,-3.042266588,0.098639861,0.503999127$ C, $0,4.3918477303,0.5192238906,-0.2766491421$ $\mathrm{H}, 0,4.280346113,1.4533486152,0.2885048398$ C, $0,5.3645025745,-0.4206223629,0.4589667897$ $\mathrm{H}, 0,5.5019057775,-1.3581888603,-0.0929926823$ $\mathrm{H}, 0,4.9856374709,-0.6736315992,1.4552647121$ C, $0,-4.3918463777,-0.5192258119,0.2766430576$ $\mathrm{H}, 0,-4.2803501442,-1.4533372644,-0.2885339861$ $\mathrm{H}, 0,-4.83667783,-0.792783141,1.2459157177$ C, $0,-5.3645096492,0.4206373116,-0.458939886$ $\mathrm{H}, 0,-4.9856545855,0.6736729166,-1.4552349701$ H,0,-6.3491757255,-0.0464945682,-0.5753151905 $\mathrm{H}, 0,-5.5019089524,1.3581893768,0.0930451143$ $\mathrm{H}, 0,4.8366897675,0.7927576832,-1.2459235212$ $\mathrm{H}, 0,-3.0279105523,1.0134811515,1.1007179481$ $\mathrm{H}, 0,6.3491682501,0.0465110835,0.5753394722$

$\mathrm{E}\left(\mathrm{B} 3 \mathrm{LYP} / 6-31 \mathrm{G}^{*}\right.$ opt $)=$ $-390.665745$ Sum of electronic and zero-point Energies $=\quad-390.432393$ Sum of electronic and thermal Energies= $\quad-390.420044$ Sum of electronic and thermal Enthalpies= $\quad-390.419100$ Sum of electronic and thermal Free Energies $=\quad-390.471728$ $\mathrm{E}\left(\mathrm{B} 3 \mathrm{LYP} / 6-311+\mathrm{G}^{* *} / / \mathrm{B} 3 \mathrm{LYP} / 6-31 \mathrm{G}^{*}\right)=\quad-390.775290$ $\chi=-81.7$

29. (RR/SS)-Undeca-3,5,7-triene (17c2, ethyl propyl hexatriene)

C, $0,-1.2436512984,0.11534181,0.2582043083$ $\mathrm{H}, 0,-1.2444012546,0.1187991932,1.3494110668$ C, $0,-0.0571854119,0.1163665629,-0.3929620418$ $\mathrm{H}, 0,-0.0564484633,0.1131371914,-1.4841781183$ C, $0,1.2365979436,0.1211799404,0.2581520628$ $\mathrm{H}, 0,1.232127923,0.1238354733,1.3498322315$ C, $0,-2.5374521391,0.1102002343,-0.3929655409$ $\mathrm{H}, 0,-2.5329792637,0.1052866688,-1.4846556501$ C, $0,2.4182622127,0.1257429502,-0.3869439372$ $\mathrm{H}, 0,2.4168084935,0.1206399458,-1.479232878$ C,0,-3.7191028742,0.1084971584,0.2519457749 C, $0,3.7671517142,0.117946916,0.2713543991$ $\mathrm{H}, 0,3.6495243675,0.1776268618,1.3618262251$ C, $0,4.6109958798,-1.1218756077,-0.088448149$ $\mathrm{H}, 0,4.706797456,-1.1876654936,-1.1812278668$ $\mathrm{H}, 0,4.0699036839,-2.0239154569,0.2257174651$ C, $0,-5.0685971067,0.1137279223,-0.406531448$ $\mathrm{H}, 0,-4.9485408772,0.0594148135,-1.4960856855$ $\mathrm{H}, 0,-5.624147244,-0.7892079359,-0.1092813145$ C, $0,-5.9103543202,1.3484403945,-0.0362186685$ $\mathrm{H}, 0,-5.4176770243,2.2703763254,-0.3639891946$ $\mathrm{H}, 0,-6.0559808158,1.4163247999,1.04856502$ 
$\mathrm{H}, 0,4.3294886677,1.0164532664,-0.030116545$

$\mathrm{H}, 0,-3.7186140166,0.1171951985,1.3443053076$

C,0,6.0035154758,-1.099637912,0.5490547716

$\mathrm{H}, 0,6.5802413737,-1.9911697829,0.2778920817$

H,0,6.574720342,-0.2210428037,0.2243932021

$\mathrm{H}, 0,5.9383671741,-1.0653514764,1.6435492218$

H,0,-6.9002709757, 1.303680996,-0.5045737563

$\mathrm{E}(\mathrm{B} 3 \mathrm{LYP} / 6-31 \mathrm{G} *$ opt $)=$

Sum of electronic and zero-point Energies=

Sum of electronic and thermal Energies=

Sum of electronic and thermal Enthalpies=

Sum of electronic and thermal Free Energies= $\mathrm{E}\left(\mathrm{B} 3 \mathrm{LYP} / 6-311+\mathrm{G}^{* *} / / \mathrm{B} 3 \mathrm{LYP} / 6-31 \mathrm{G}^{*}\right)=$ $\chi=-91.8$
$-429.979673$

$-429.717715$

$-429.703997$

$-429.703053$

$-429.759280$

$-430.099790$

30. (RR/SS)-Dodeca-4,6,8-triene (17c3, dipropyl hexatriene)

$\mathrm{C}, 0,-0.5934277674,0.0012926385,0.3251638745$

$\mathrm{H}, 0,-0.594556263,0.0063829336,1.4163852488$

C, $0,0.5934797093,-0.0012109884,-0.3252589401$

$\mathrm{H}, 0,0.5946087715,-0.006301595,-1.4164803024$

$\mathrm{C}, 0,1.8868736418,0.0028956368,0.326523898$

$\mathrm{H}, 0,1.8821098779,0.008100847,1.418193221$

$\mathrm{C}, 0,-1.8868224513,-0.0028139796,-0.3266176339$

$\mathrm{H}, 0,-1.8820604197,-0.0080148618,-1.4182868762$

C,0,3.0689855441,0.0048796852,-0.3177909592

$\mathrm{H}, 0,3.0682323107,-0.0026883701,-1.4100519832$

C, $0,-3.0689323744,-0.0047980616,0.3177005066$

$\mathrm{C}, 0,4.4174040584,0.0000307225,0.3414207103$

$\mathrm{H}, 0,4.2991701428,0.0625769119,1.4316950181$

C,0,5.2638455527,-1.2393144684,-0.0142966912

H, $0,5.3587652436,-1.3093606169,-1.1068985558$

$\mathrm{H}, 0,4.7258724496,-2.1416210022,0.3043676956$

C,0,-4.4173554831,0.0000251948,-0.3415021573

$\mathrm{H}, 0,-4.2991200851,-0.0623361526,-1.4317870756$

$\mathrm{H}, 0,-4.9783573082,-0.8986368819,-0.038025203$

C,0,-5.2639205979,1.2392232704,0.0144214809

$\mathrm{H}, 0,-4.7260638486,2.1416362433,-0.3041389054$

H, $0,-5.358803305,1.3091072873,1.1070371549$

H,0,4.978499036,0.8985841975,0.0377990518

$\mathrm{H}, 0,-3.0681753734,0.002772265,1.4099617781$

C,0,6.6570159281,-1.2103490383,0.6217394012

$\mathrm{H}, 0,7.2366442563,-2.1006481016,0.3526665498$

$\mathrm{H}, 0,7.2246291141,-0.3307057443,0.2938536984$

H,0,6.5926735026,-1.1731036713,1.7161959114

C,0,-6.6571139596,1.2101926282,-0.6215612386

$\mathrm{H}, 0,-7.2246080601,0.3304295532,-0.2937903009$

$\mathrm{H}, 0,-7.2368373115,2.1003797991,-0.3523232883$

H,0,-6.5928135339,1.1731275196,-1.7160263437

$\mathrm{E}\left(\mathrm{B} 3 \mathrm{LYP} / 6-31 \mathrm{G}^{*}\right.$ opt $)=$

$-469.293573$

Sum of electronic and zero-point Energies=

Sum of electronic and thermal Energies=

$-469.003091$

$-468.987993$

Sum of electronic and thermal Enthalpies=

$-468.987049$

Sum of electronic and thermal Free Energies=

$-469.047000$ 
$\mathrm{E}\left(\mathrm{B} 3 \mathrm{LYP} / 6-311+\mathrm{G}^{* *} / / \mathrm{B} 3 \mathrm{LYP} / 6-31 \mathrm{G}^{*}\right)=$

$-469.424258$

$\chi=-102.0$

31. (RR/SS)-Dodeca-3,5,7,9-tetraene (17d1, diethyl octatetraene)

$\mathrm{C}, 0,-4.2762897574,0.0422623814,0.6041517152$

$\mathrm{H}, 0,-4.2045843512,0.0112946389,1.693688071$

C, $0,-3.1383854338,0.0426863362,-0.1169193754$

$\mathrm{H}, 0,-3.2055237752,0.0703820617,-1.206199294$

C, $0,-1.8073676646,0.0116493502,0.4478371621$

$\mathrm{H}, 0,-1.7361813864,-0.0166023281,1.5362586562$

C, $0,-5.6654182209,0.0648053565,0.0353568114$

$\mathrm{H}, 0,-6.1864949521,0.9718765859,0.3790473623$

C, $0,-0.663026498,0.0155775159,-0.2806748765$

C, $0,-6.500204295,-1.1619414981,0.4459955092$

C, $0,0.6629897156,-0.015548019,0.2807741293$

$\mathrm{H}, 0,-6.5785764665,-1.2388822376,1.5370940202$

$\mathrm{H}, 0,-0.7354543478,0.0443196361,-1.369116273$

$\mathrm{H}, 0,0.7354198792,-0.0442917348,1.3692153376$

C, $0,1.8073323787,-0.0116153664,-0.4477415194$

$\mathrm{H}, 0,1.7361441996,0.0166276502,-1.536162994$

C, $0,3.1383527444,-0.04263595,0.1170131502$

H,0,3.2054938567,-0.0703246321, 1.2062929522

C,0,4.2762516117,-0.042196403,-0.6040623555

$\mathrm{H}, 0,4.2045403299,-0.0112393875,-1.6935986285$

C,0,5.6653827762,-0.0647360872,-0.0352782511

H,0,6.1863318902,-0.9720101216,-0.3786361304

C, $0,6.5003470069,1.1617426444,-0.4463612203$

$\mathrm{H}, 0,6.0431361499,2.0873286641,-0.0798512193$

$\mathrm{H}, 0,6.5787567072,1.238257633,-1.5374866151$

$\mathrm{H}, 0,-6.0428723535,-2.0873236745,0.0791240563$

$\mathrm{H}, 0,5.6160610696,-0.1310356136,1.0591118178$

$\mathrm{H}, 0,-5.6161064804,0.1315055951,-1.059008155$

$\mathrm{H}, 0,7.5168268681,1.0979320977,-0.0415152017$

H,0,-7.5167030206,-1.0981163976,0.0411969616

$\mathrm{E}\left(\mathrm{B} 3 \mathrm{LYP} / 6-31 \mathrm{G}^{*}\right.$ opt $)=$

$-468.073003$

Sum of electronic and zero-point Energies=

Sum of electronic and thermal Energies=

Sum of electronic and thermal Enthalpies=

Sum of electronic and thermal Free Energies=

$\mathrm{E}\left(\mathrm{B} 3 \mathrm{LYP} / 6-311+\mathrm{G}^{* *} / / \mathrm{B} 3 \mathrm{LYP} / 6-31 \mathrm{G}^{*}\right)=$

$-467.806063$

$-467.791503$

$-467.790558$

$-467.848888$

$\chi=-93.6$

$-468.202871$

32. (RR/SS)-Trideca-3,5,7,9-tetraene (17d2, ethyl propyl octatetraene)

C,0,-4.9653207,-0.1057444534,0.2627936528

$\mathrm{H}, 0,-4.9652485501,-0.1009184122,1.355090604$

C,0,-3.7824458946,-0.1094550937,-0.3819226563

$\mathrm{H}, 0,-3.7783109042,-0.1176723219,-1.4735535033$

C, $0,-2.4910638734,-0.1008124907,0.2690514073$

H, $0,-2.4907146759,-0.0931521016,1.360127581$

C,0,-6.3138115072,-0.1253721736,-0.3967431928

$\mathrm{H}, 0,-6.8717003555,0.7813685338,-0.115703495$

C, $0,-1.3016787439,-0.1022353999,-0.3834593474$

C, $0,-7.1532955718,-1.3553511065,-0.0056086683$ 
C, $0,-0.0149351096,-0.0952826566,0.2635780333$

$\mathrm{H}, 0,-7.3018535184,-1.4032725587,1.0798374092$

$\mathrm{H}, 0,-1.3034291526,-0.1094213148,-1.474656808$

$\mathrm{H}, 0,-0.0132970923,-0.0886389385,1.354778707$

C, $0,1.1746145341,-0.0974401171,-0.3886708284$

$\mathrm{H}, 0,1.1746026127,-0.10449978,-1.479756693$

C, $0,2.4656686451,-0.0920013902,0.2628560375$

$\mathrm{H}, 0,2.4609009209,-0.0854237479,1.3544838032$

C, $0,3.6490524894,-0.0988924049,-0.3811511636$

$\mathrm{H}, 0,3.648954793,-0.1031376934,-1.4733510234$

C, $0,4.9962530976,-0.0837407113,0.2796888962$

$\mathrm{H}, 0,5.5606611535,-0.9832681398,-0.0149683379$

C,0,5.839262846,1.1551808882,-0.0862463876

$\mathrm{H}, 0,5.2961363625,2.0586162142,0.2202152149$

$\mathrm{H}, 0,5.938684311,1.2135004668,-1.1791005073$

C,0,7.2295319108,1.1388260817,0.5564134907

$\mathrm{H}, 0,7.8025709082,0.2582683437,0.2406166132$

$\mathrm{H}, 0,7.8065056954,2.0287730328,0.2806213783$

$\mathrm{H}, 0,7.1601867534,1.1129464764,1.6508747875$

$\mathrm{H}, 0,-6.6574531121,-2.2819817896,-0.3148569781$

$\mathrm{H}, 0,4.876586474,-0.1365925016,1.3702915811$

$\mathrm{H}, 0,-6.1928334325,-0.0897279008,-1.486963056$

H,0,-8.1419419249,-1.3218396976,-0.4775029178

$\mathrm{E}\left(\mathrm{B} 3 \mathrm{LYP} / 6-31 \mathrm{G}^{*} \mathrm{opt}\right)=$

$-507.386890$

Sum of electronic and zero-point Energies=

Sum of electronic and thermal Energies=

Sum of electronic and thermal Enthalpies=

Sum of electronic and thermal Free Energies=

$\mathrm{E}\left(\mathrm{B} 3 \mathrm{LYP} / 6-311+\mathrm{G}^{* *} / / \mathrm{B} 3 \mathrm{LYP} / 6-31 \mathrm{G}^{*}\right)=$

$-507.091466$

$-507.075507$

$-507.074563$

$-507.136822$

$\chi=-103.7$

$-507.527318$

33. (RR/SS)-Tetradeca-4,6,8,10-tetraene (17d3, dipropyl octatetraene)

C, $0,-4.266523546,-0.5190258401,0.4241600627$

$\mathrm{H}, 0,-4.2259807737,-1.6060560464,0.3257252122$

C, $0,-3.1152786062,0.1772419913,0.3534067947$

$\mathrm{H}, 0,-3.1510730432,1.2641954626,0.4480050505$

C, $0,-1.8083572388,-0.4115084908,0.1620170959$

$\mathrm{H}, 0,-1.7675708665,-1.4978016865,0.0680681209$

C, $0,-5.6304723778,0.0768418509,0.6153975326$

$\mathrm{H}, 0,-6.0726444753,-0.3082595733,1.5485998588$

C, $0,-0.6509137983,0.2929652476,0.0945221693$

C, $0,-6.5998932914,-0.2374023463,-0.5422340276$

C, $0,0.6515978839,-0.2916075409,-0.0953323734$

C, $0,-8.0021328291,0.3357510508,-0.315544192$

$\mathrm{H}, 0,-8.4540580694,-0.0709741231,0.5976488657$

$\mathrm{H}, 0,-7.9725204826,1.4273639551,-0.2117101736$

H, $0,-6.6636803382,-1.3266407395,-0.6732066147$

$\mathrm{H}, 0,-0.6932585545,1.3792643538,0.189023855$

$\mathrm{H}, 0,0.693961738,-1.3779154388,-0.1897236008$

C, $0,1.809013297,0.4128954872,-0.1630027723$

H, $0,1.7681903396,1.4992004768,-0.0692059117$

C, $0,3.1159454244,-0.1758178706,-0.3544243612$

$\mathrm{H}, 0,3.1518204563,-1.2628030949,-0.4486497945$

C, $0,4.267111703,0.5205248112,-0.4257685883$ 
$\mathrm{H}, 0,4.226483681,1.6075988275,-0.3278959281$

C,0,5.6311029526,-0.0754058168,-0.6164755708

$\mathrm{H}, 0,6.0753659047,0.3128348367,-1.5473526045$

C, $0,6.598383765,0.2341541712,0.5442636079$

$\mathrm{H}, 0,6.176651757,-0.1655260596,1.475700278$

$\mathrm{H}, 0,6.6622130622,1.3228603758,0.6795420309$

C, $0,8.0008924133,-0.3385517772,0.3180924892$

$\mathrm{H}, 0,8.4543768575,0.0709285958,-0.593093857$

H,0,8.6667896525,-0.1045007459,1.1563695299

H,0,7.9712601497,-1.4298318777,0.210817018

$\mathrm{H}, 0,-6.1801238251,0.1588225108,-1.4760373957$

H,0,5.5472745969,-1.1631770243,-0.7438435215

$\mathrm{H}, 0,-5.5469723419,1.1651031352,0.7387507129$

H,0,-8.6693599351,0.0989843154,-1.1519983326

$\mathrm{E}\left(\mathrm{B} 3 \mathrm{LYP} / 6-31 \mathrm{G}^{*}\right.$ opt $)=$

Sum of electronic and zero-point Energies=

Sum of electronic and thermal Energies=

Sum of electronic and thermal Enthalpies=

Sum of electronic and thermal Free Energies=

$\mathrm{E}\left(\mathrm{B} 3 \mathrm{LYP} / 6-311+\mathrm{G}^{* *} / / \mathrm{B} 3 \mathrm{LYP} / 6-31 \mathrm{G}^{*}\right)=$

$\chi=-113.8$
$-546.700850$

$-546.376702$

$-546.359469$

$-546.358525$

$-546.423645$

$-546.851871$

34. (RR/SS)-Tetradeca-3,5,7,9,11-pentaene (17e1, diethyl decapentaene)

C,0,4.3540194924,-0.3460459611,-0.2076348265

$\mathrm{H}, 0,4.3564380773,-1.4095195581,0.0387079082$

C, $0,3.0624534898,0.2991598327,-0.2727626851$

$\mathrm{H}, 0,3.0552000302,1.3616121844,-0.520839103$

C, $0,1.8783242918,-0.3265516169,-0.0481014814$

$\mathrm{H}, 0,1.8873973749,-1.3891769615,0.199799659$

C, $0,5.5308714745,0.2715881462,-0.4304869993$

H, $0,5.5244453175,1.3366336481,-0.6727687489$

C, $0,0.5929063616,0.3135833697,-0.1127273005$

C, $0,6.8808779024,-0.3807459348,-0.3576360908$

C, $0,-0.5929064889,-0.3135020812,0.1128126505$

C, $0,7.7943538988,0.2497496317,0.7095704208$

$\mathrm{H}, 0,7.9381049687,1.3209265032,0.5241492963$

$\mathrm{H}, 0,7.3610356915,0.140171489,1.7097093788$

$\mathrm{H}, 0,7.3793526877,-0.2986110685,-1.3359524451$

$\mathrm{H}, 0,0.5829219488,1.3760091849,-0.3610759587$

$\mathrm{H}, 0,-0.582919391,-1.3759279306,0.3611612612$

$\mathrm{C}, 0,-1.8783288464,0.3266226305,0.0481725151$

$\mathrm{H}, 0,-1.8874109711,1.3892464543,-0.1997346437$

C, $0,-3.0624526498,-0.2991058371,0.2728146796$

$\mathrm{H}, 0,-3.0551849977,-1.3615566889,0.5208971506$

C, $0,-4.3540311946,0.3460709546,0.2076462293$

$\mathrm{H}, 0,-4.3564694858,1.4095414725,-0.038708753$

C, $0,-5.5308734194,-0.2715931078,0.4304663962$

$\mathrm{H}, 0,-5.5244252186,-1.3366358487,0.6727611544$

C, $0,-6.8808976454,0.3806928363,0.3575584938$

$\mathrm{H}, 0,-6.7630370449,1.453412047,0.1581368575$

$\mathrm{H}, 0,-7.3793965903,0.2985705654,1.3358644124$

C, $0,-7.7943221314,-0.2498734234,-0.7096515419$

$\mathrm{H}, 0,-7.938025774,-1.3210524869,-0.5242057528$

H,0,-7.360989202,-0.1403002259,-1.7097847356 
$\mathrm{H}, 0,6.762986454,-1.4534679486,-0.1582466783$

$\mathrm{H}, 0,-8.7820155035,0.2251805002,-0.7101860218$

$\mathrm{H}, 0,8.7820244168,-0.2253519672,0.7100730048$

$\mathrm{E}\left(\mathrm{B} 3 \mathrm{LYP} / 6-31 \mathrm{G}^{*}\right.$ opt $)=$

Sum of electronic and zero-point Energies=

Sum of electronic and thermal Energies=

Sum of electronic and thermal Enthalpies=

Sum of electronic and thermal Free Energies= $\mathrm{E}\left(\mathrm{B} 3 \mathrm{LYP} / 6-311+\mathrm{G}^{* *} / / \mathrm{B} 3 \mathrm{LYP} / 6-31 \mathrm{G}^{*}\right)=$ $\chi=-105.4$
$-545.480510$

$-545.179990$

$-545.163252$

$-545.162308$

$-545.226177$

$-545.630672$

35. (RR/SS)-Pentadeca-3,5,7,9,11-pentaene (17e2, ethyl propyl decapentaene)

C,0,-5.0252609248,0.2708188552,-0.2983405375

$\mathrm{H}, 0,-5.0421790069,1.3623315961,-0.2983300098$

C, $0,-3.7261596631,-0.355239067,-0.203421651$

$\mathrm{H}, 0,-3.7045859671,-1.4460747896,-0.2032367228$

C, $0,-2.5514715354,0.3205047596,-0.1159346445$

$\mathrm{H}, 0,-2.5747699108,1.411455285,-0.1166590379$

C, $0,-6.1925022855,-0.3967660296,-0.3877907355$

$\mathrm{H}, 0,-6.1713715501,-1.4888207741,-0.3841735557$

C, $0,-1.2587739375,-0.3007286074,-0.0201205209$

$\mathrm{C}, 0,-7.5504532133,0.236754707,-0.4766372673$

$\mathrm{C}, 0,-0.0824727137,0.3765832624,0.0685864904$

C, $0,-8.4692937569,-0.1506206199,0.6967022038$

$\mathrm{H}, 0,-8.5978894693,-1.2380720692,0.7552827569$

$\mathrm{H}, 0,-8.0492073791,0.1866459727,1.650567807$

H, $,-8.0361347691,-0.0702131307,-1.4158656474$

$\mathrm{H}, 0,-1.2346367646,-1.3915692517,-0.0187192486$

$\mathrm{H}, 0,-0.1066953609,1.4674273337,0.0671228995$

C, $0,1.210071784,-0.244428977,0.1675980025$

$\mathrm{H}, 0,1.2335144387,-1.3353686296,0.1701635825$

C, $0,2.384292531,0.4316990844,0.2587888644$

$\mathrm{H}, 0,2.3624094232,1.522536873,0.2566896029$

C, $0,3.6828887157,-0.1936860593,0.3634588491$

$\mathrm{H}, 0,3.7001265755,-1.2851718906,0.3671036829$

C, $0,4.8491258603,0.4747527708,0.46117786$

$\mathrm{H}, 0,4.8266283227,1.5666721947,0.4548349376$

C, $0,6.2057183619,-0.1577927657,0.5648285772$

$\mathrm{H}, 0,6.1038380019,-1.2495121376,0.6297548516$

$\mathrm{H}, 0,6.6908781167,0.1653738674,1.5000320621$

C, $0,7.1377312394,0.2032872508,-0.6103656342$

$\mathrm{H}, 0,7.2120741949,1.2968837601,-0.6886121161$

$\mathrm{H}, 0,6.6794513734,-0.1399737989,-1.5469326337$

C, $0,8.5387832495,-0.3980023521,-0.4630935768$

$\mathrm{H}, 0,9.1789757133,-0.1287438391,-1.3107819669$

$\mathrm{H}, 0,9.0286148957,-0.0422357332,0.4517586952$

$\mathrm{H}, 0,8.497271524,-1.4929430505,-0.411608887$

$\mathrm{H}, 0,-7.4470945726,1.3283568098,-0.5228102681$

$\mathrm{H}, 0,-9.4625600987,0.2981981281,0.5818015378$

$\mathrm{E}\left(\mathrm{B} 3 \mathrm{LYP} / 6-31 \mathrm{G}^{*}\right.$ opt $)=$

$-584.794421$

Sum of electronic and zero-point Energies=

$-584.465309$

Sum of electronic and thermal Energies=

$-584.447208$

Sum of electronic and thermal Enthalpies=

$-584.446263$ 
Sum of electronic and thermal Free Energies= $\mathrm{E}\left(\mathrm{B} 3 \mathrm{LYP} / 6-311+\mathrm{G}^{* *} / / \mathrm{B} 3 \mathrm{LYP} / 6-31 \mathrm{G}^{*}\right)=$ $\chi=-115.5$
$-584.513805$

$-584.955186$

36. (RR/SS)-Hexadeca-4,6,8,10,12-pentaene (17e3, dipropyl decapentaene)

C,0,-4.3713075896,0.1026488502,0.066399289

$\mathrm{H}, 0,-4.4301382255,0.1154807388,1.1563768778$

C, $0,-3.0461505562,0.0669195441,-0.5090183329$

$\mathrm{H}, 0,-2.9832461717,0.0531253652,-1.5981831527$

C, $0,-1.894895424,0.0481692054,0.21108601$

$\mathrm{H}, 0,-1.9592090191,0.0624786181,1.3002959457$

C, $0,-5.5150448297,0.1229571234,-0.6463137558$

$\mathrm{H}, 0,-5.4505836674,0.1076201575,-1.7364921768$

C, $0,-0.5766236048,0.0098475246,-0.3606165441$

C, $0,-6.8987706843,0.1422303948,-0.0663760689$

C, $0,0.5765299121,-0.0096342567,0.3606384769$

C,0,-7.7341736233,-1.0961474766,-0.4516907548

$\mathrm{H}, 0,-7.7622332995,-1.1853714045,-1.5465843955$

H,0,-7.2252201212,-1.9967869965,-0.0844201005

C, $0,-9.1633817503,-1.043836837,0.0966827452$

$\mathrm{H}, 0,-9.704591969,-0.1704740812,-0.2878458346$

H,0,-9.1659536365,-0.9782947571,1.1917147128

H,0,-7.4328313748, 1.0403411556,-0.4164581562

H,0,-0.5116814706,-0.0047588942,-1.4497029358

$\mathrm{H}, 0,0.5115942228,0.0049563471,1.4497251836$

C, $0,1.8947947438,-0.0479215651,-0.2110823164$

H,0,1.9590879276,-0.062125006,-1.3002951905

C,0,3.0460647378,-0.066751844,0.508990582

$\mathrm{H}, 0,2.983194437,-0.0530953765,1.598158823$

C,0,4.3712016596,-0.1023885041,-0.0664853331

H,0,4.4299808295,-0.1149789622,-1.1564680281

C, $0,5.5149673274,-0.1228472625,0.6461724735$

H,0,5.4505507355,-0.1077557873,1.7363588912

C,0,6.8986689136,-0.1421559392,0.0661748496

H,0,6.8429472559,-0.2186784448,-1.0280894641

$\mathrm{H}, 0,7.4319527847,-1.0414408843,0.4144346783$

C,0,7.7351882831,1.0946606054,0.4539964962

H,0,7.7661881504,1.1796202581,1.549151291

H,0,7.2253693046,1.9967747699,0.0915710348

C,0,9.162921454,1.0443414585,-0.0983773621

$\mathrm{H}, 0,9.7319686907,1.9364337737,0.1867128909$

H,0,9.7043261827,0.1681890266,0.2794910508

H,0,9.1626024127,0.9853889721,-1.1937810471

H,0,-9.7308721747,-1.9381633659,-0.1844861045

H,0,-6.8431356184,0.2209686511,1.0277324806

$\mathrm{E}\left(\mathrm{B} 3 \mathrm{LYP} / 6-31 \mathrm{G}^{*} \mathrm{opt}\right)=$

$-624.108347$

Sum of electronic and zero-point Energies=

$-623.750702$

Sum of electronic and thermal Energies=

$-623.731223$

Sum of electronic and thermal Enthalpies=

$-623.730279$

Sum of electronic and thermal Free Energies=

$-623.801456$

$\mathrm{E}\left(\mathrm{B} 3 \mathrm{LYP} / 6-311+\mathrm{G}^{* *} / / \mathrm{B} 3 \mathrm{LYP} / 6-31 \mathrm{G}^{*}\right)=$ $-624.279647$

$\chi=-125.6$

37. Bicyclo[4.3.0]nona-1(6),2,8-triene, held planar (NImag=1) $(\mathbf{1 8})$ 


$\begin{array}{lrrr}6 & 0.318604 & 0.715325 & 0.000000 \\ 6 & 0.297949 & -0.640367 & 0.000000 \\ 6 & -0.959170 & -1.462952 & 0.000000 \\ 6 & -2.273418 & -0.611430 & 0.000000 \\ 6 & -2.101717 & 0.892808 & 0.000000 \\ 6 & -0.909711 & 1.506623 & 0.000000 \\ 6 & 1.716127 & 1.176611 & 0.000000 \\ 6 & 2.540198 & 0.110323 & 0.000000 \\ 6 & 1.713695 & -1.154887 & 0.000000 \\ 1 & -0.956110 & -2.135390 & 0.870661 \\ 1 & -2.888232 & -0.884010 & 0.869475 \\ 1 & -3.019099 & 1.478880 & 0.000000 \\ 1 & -0.843037 & 2.592411 & 0.000000 \\ 1 & 2.014237 & 2.220926 & 0.000000 \\ 1 & 1.928602 & -1.784960 & -0.877764 \\ 1 & 1.928601 & -1.784958 & 0.877767 \\ 1 & 3.624042 & 0.124178 & 0.000001 \\ 1 & -2.888232 & -0.884010 & -0.869474 \\ 1 & -0.956111 & -2.135390 & -0.870662\end{array}$

$\mathrm{E}\left(\mathrm{B} 3 \mathrm{LYP} / 6-31 \mathrm{G}^{*}\right.$ opt $)=$

$-348.929387$

Sum of electronic and zero-point Energies $=\quad-348.766660$

Sum of electronic and thermal Energies $=\quad-348.760044$

Sum of electronic and thermal Enthalpies= $\quad-348.759099$

Sum of electronic and thermal Free Energies $=\quad-348.797500$

$\mathrm{E}\left(\mathrm{B} 3 \mathrm{LYP} / 6-311+\mathrm{G}^{* *} / / \mathrm{B} 3 \mathrm{LYP} / 6-31 \mathrm{G}^{*}\right)=\quad-349.019719$

$\chi=-65.2$

38. Bicyclo[4.3.0]nona-2,6,9-triene, held planar $(\mathrm{NImag=1)}(\mathbf{1 9})$

$\begin{array}{rrrr}6 & 0.356546 & 0.758035 & 0.000000 \\ 6 & 0.312124 & -0.719275 & 0.000000 \\ 6 & -0.995067 & -1.476796 & 0.000000 \\ 6 & -2.276709 & -0.587670 & 0.000000 \\ 6 & -2.070178 & 0.905534 & 0.000000 \\ 6 & -0.876611 & 1.521899 & 0.000000 \\ 6 & 1.648044 & 1.166319 & -0.000001 \\ 6 & 2.548936 & -0.039748 & 0.000000 \\ 6 & 1.574900 & -1.191598 & 0.000000 \\ 1 & -1.022410 & -2.142139 & 0.871798 \\ 1 & -2.900028 & -0.843721 & 0.868990 \\ 1 & -2.979892 & 1.504415 & 0.000000 \\ 1 & -0.817746 & 2.608340 & 0.000000 \\ 1 & 2.002246 & 2.191483 & -0.000001 \\ 1 & 3.215066 & -0.059482 & 0.877530 \\ 1 & 1.878227 & -2.233756 & 0.000000 \\ 1 & 3.215067 & -0.059483 & -0.877529 \\ 1 & -2.900028 & -0.843721 & -0.868991 \\ 1 & -1.022410 & -2.142138 & -0.871798\end{array}$

$\mathrm{E}\left(\mathrm{B} 3 \mathrm{LYP} / 6-31 \mathrm{G}^{*}\right.$ opt $)=$

$-348.933498$

Sum of electronic and zero-point Energies $=\quad-348.770491$

Sum of electronic and thermal Energies $=\quad-348.763970$

Sum of electronic and thermal Enthalpies $=\quad-348.763026$

Sum of electronic and thermal Free Energies $=\quad-348.801228$ 
39. Tropylium-indene cation (20)

C,0,-2.3052987283,-0.0825129347,-0.3187503409

C, $0,-2.3156286039,0.0443704643,1.0825058545$

C, $0,-1.238548798,0.1488582902,1.955871963$

C, $0,-1.2217897321,-0.1359588452,-1.1860908201$

C, $0,0.1365315455,0.1541380487,1.6601664028$

H,0,-3.2842094996,-0.1478192713,-0.7875190608

$\mathrm{H}, 0,-3.3004892308,0.063065036,1.5423912605$

$\mathrm{H}, 0,-1.4898492439,0.2386373646,3.0097603895$

H,0,-1.4599274645,-0.2373247048,-2.2425914919

$\mathrm{H}, 0,0.8013054851,0.2474977134,2.5168223272$

C, $0,0.1483611683,-0.0755768633,-0.870916032$

C, $0,1.2077862422,-0.14104825,-1.8597953805$

$\mathrm{H}, 0,1.0317054999,-0.2418310259,-2.9244624533$

C, $0,0.7474235323,0.0557643472,0.4212106666$

C,0,2.2454104587,0.0734462808,0.2326960205

H,0,2.6923602953,1.00017803,0.6238338839

C,0,2.4120909672,-0.0575297899,-1.2473057384

$\mathrm{H}, 0,3.3781853673,-0.0800856339,-1.7385540198$

$\mathrm{H}, 0,2.73289048,-0.7460219963,0.7827635913$

$\mathrm{E}\left(\mathrm{B} 3 \mathrm{LYP} / 6-31 \mathrm{G}^{*}\right.$ opt $)=$

$-386.215941$

Sum of electronic and zero-point Energies= Sum of electronic and thermal Energies= Sum of electronic and thermal Enthalpies= Sum of electronic and thermal Free Energies= $\mathrm{E}\left(\mathrm{B} 3 \mathrm{LYP} / 6-311+\mathrm{G}^{* *} / / \mathrm{B} 3 \mathrm{LYP} / 6-31 \mathrm{G}^{*}\right)=$ $\chi=-75.8$

$-386.056541$

$-386.049127$

$-386.048183$

$-386.088456$

$-386.303464$

40. Tropylium-isoindene cation (21)

C,0,-2.3086400284,0.0862161385,-0.3506517337

C, $0,-2.2943206073,0.1123347513,1.057173946$

C, $0,-1.233448822,0.0862161386,1.982770643$

C, $0,-1.2276002628,0.0258751143,-1.213008132$

C,0,0.1244695841,0.0258751144,1.721307194

H,0,-3.2917952237,0.1171480787,-0.8105423734

H,0,-3.2821385441,0.1607222537, 1.5123393588

$\mathrm{H}, 0,-1.5226463738,0.1171480789,3.0289342604$

$\mathrm{H}, 0,-1.4597054727,0.0146284976,-2.2765304527$

$\mathrm{H}, 0,0.7821122971,0.0146284979,2.5887509196$

C, $0,0.1567454278,-0.023338788,-0.894792791$

C,0,1.1591746099,-0.0800898872,-1.8315759825

H,0,1.029768444,-0.0940726538,-2.9073124206

C, $0,0.7820302515,-0.0233387879,0.4622250033$

C, $0,2.1454491423,-0.0800898871,0.3088759199$

C,0,2.475611364,-0.1194370333,-1.1407088557

H,0,3.060247666,-1.0169405007,-1.4100967801

H,0,2.8791035807,-0.0940726536,1.1061876564

H,0,3.1282296724,0.7194731596,-1.4414214371

$\mathrm{E}\left(\mathrm{B} 3 \mathrm{LYP} / 6-31 \mathrm{G}^{*}\right.$ opt $)=$

$-386.178797$ 
Sum of electronic and zero-point Energies= Sum of electronic and thermal Energies= Sum of electronic and thermal Enthalpies= Sum of electronic and thermal Free Energies= $\mathrm{E}\left(\mathrm{B} 3 \mathrm{LYP} / 6-311+\mathrm{G}^{* *} / / \mathrm{B} 3 \mathrm{LYP} / 6-31 \mathrm{G}^{*}\right)=$ $\chi=-56.5$
$-386.021283$

$-386.013684$

$-386.012740$

$-386.053321$

$-386.267060$

41. 7,8-Dihydrotropylium-indene cation, held planar (NImag=1) (22)

$\begin{array}{lrrr}6 & -1.889264 & 1.412257 & 0.000004 \\ 6 & -2.546078 & 0.215197 & -0.000127 \\ 6 & -2.079929 & -1.199443 & -0.000030 \\ 6 & -0.492088 & 1.640494 & 0.000118 \\ 6 & -0.583422 & -1.626142 & 0.000116 \\ 1 & -2.505634 & 2.306526 & -0.000011 \\ 1 & -3.634079 & 0.283514 & -0.000279 \\ 1 & -2.584351 & -1.665366 & -0.859999 \\ 1 & -0.209447 & 2.693107 & 0.000226 \\ 1 & -0.406242 & -2.286632 & -0.861137 \\ 6 & 0.589841 & 0.747365 & 0.000008 \\ 6 & 2.003481 & 1.174072 & -0.000029 \\ 1 & 2.311842 & 2.213296 & -0.000042 \\ 6 & 0.545190 & -0.651855 & 0.000026 \\ 6 & 1.960502 & -1.153684 & -0.000035 \\ 1 & 2.144063 & -1.799824 & 0.873452 \\ 6 & 2.799789 & 0.095309 & -0.000062 \\ 1 & 3.882552 & 0.085359 & -0.000106 \\ 1 & 2.143977 & -1.799790 & -0.873568 \\ 1 & -0.406328 & -2.286314 & 0.861637 \\ 1 & -2.584493 & -1.665288 & 0.859898\end{array}$

$\mathrm{E}\left(\mathrm{B} 3 \mathrm{LYP} / 6-31 \mathrm{G}^{*}\right.$ opt $)=$

Sum of electronic and zero-point Energies= Sum of electronic and thermal Energies= Sum of electronic and thermal Enthalpies= Sum of electronic and thermal Free Energies= $\mathrm{E}\left(\mathrm{B} 3 \mathrm{LYP} / 6-311+\mathrm{G}^{* *} / / \mathrm{B} 3 \mathrm{LYP} / 6-31 \mathrm{G}^{*}\right)=$ $\chi=-55.1$
-387.374298
-387.194069
-387.186296
-387.185352
-387.226831
-387.465117

42. 7,8-Dihydro-2H-tropylium-isoindene cation, held planar $(\mathrm{NImag}=1)(\mathbf{2 3})$

$\begin{array}{lrrr}6 & -2.079644 & -1.173370 & -0.000216 \\ 6 & -2.511587 & 0.241301 & -0.000854 \\ 6 & -1.849701 & 1.448428 & -0.000217 \\ 6 & -0.620081 & -1.688723 & 0.000909 \\ 6 & -0.463914 & 1.666421 & 0.000814 \\ 1 & -2.619598 & -1.617951 & 0.853726 \\ 1 & -3.598879 & 0.326785 & -0.001781 \\ 1 & -2.471206 & 2.339348 & -0.000488 \\ 1 & -0.512159 & -2.350396 & -0.866125 \\ 1 & -0.151011 & 2.709148 & 0.001428 \\ 6 & 0.565890 & -0.753096 & 0.000259 \\ 6 & 1.851009 & -1.174261 & -0.000389 \\ 1 & 2.177518 & -2.208424 & -0.000642 \\ 6 & 0.583915 & 0.723742 & 0.000390\end{array}$




\begin{tabular}{|c|c|c|c|}
\hline 6 & 1.900575 & 1.154094 & 0.000047 \\
\hline 6 & 2.809882 & -0.016042 & -0.000627 \\
\hline 1 & 3.483469 & 0.002902 & 0.872114 \\
\hline 1 & 2.222313 & 2.190811 & 0.000141 \\
\hline 1 & 3.482621 & 0.003215 & -0.874012 \\
\hline 1 & -2.618346 & -1.618243 & -0.854803 \\
\hline 1 & -0.512785 & -2.348170 & 0.869743 \\
\hline
\end{tabular}

$\mathrm{E}\left(\mathrm{B} 3 \mathrm{LYP} / 6-31 \mathrm{G}^{*}\right.$ opt $)=$

Sum of electronic and zero-point Energies=

Sum of electronic and thermal Energies=

Sum of electronic and thermal Enthalpies=

Sum of electronic and thermal Free Energies= $\mathrm{E}\left(\mathrm{B} 3 \mathrm{LYP} / 6-311+\mathrm{G}^{* *} / / \mathrm{B} 3 \mathrm{LYP} / 6-31 \mathrm{G}^{*}\right)=$ $\chi=-65.6$
$-387.375898$

$-387.195413$

$-387.187784$

$-387.186840$

$-387.227866$

$-387.467116$

43. 11,12-Dihydro[11] annulenium mono-trans planar-indene cation (NImag=3)

$\begin{array}{rrrr}6 & -2.157731 & 0.199595 & 0.000003 \\ 6 & -2.951523 & -0.962108 & 0.000006 \\ 6 & -2.392258 & -2.194430 & 0.000001 \\ 6 & -0.980186 & -2.667716 & -0.000007 \\ 6 & 0.473809 & -2.005076 & -0.000014 \\ 6 & 1.328487 & -0.691221 & -0.000017 \\ 6 & 1.442544 & 0.716622 & -0.000015 \\ 6 & 0.722948 & 1.995540 & -0.000009 \\ 6 & -0.451756 & 2.747687 & -0.000002 \\ 6 & -1.855947 & 2.597985 & 0.000005 \\ 6 & -2.674551 & 1.473872 & 0.000008 \\ 1 & -4.033800 & -0.864337 & 0.000016 \\ 1 & -3.078078 & -3.042061 & 0.000014 \\ 1 & -0.958164 & -3.358580 & -0.855885 \\ 1 & 0.925644 & -2.510791 & -0.857017 \\ 1 & 1.509880 & 2.741806 & -0.000007 \\ 1 & -0.200895 & 3.805342 & -0.000001 \\ 1 & -2.380015 & 3.551986 & 0.000008 \\ 1 & -3.751656 & 1.616913 & 0.000015 \\ 1 & -1.082681 & 0.074053 & -0.000002 \\ 6 & 2.916037 & 1.031468 & -0.000006 \\ 6 & 2.769295 & -1.234264 & 0.000029 \\ 6 & 3.673392 & -0.061145 & 0.000008 \\ 1 & 3.281491 & 2.052216 & -0.000007 \\ 1 & 2.935480 & -1.879934 & -0.874574 \\ 1 & 4.754451 & -0.118183 & 0.000002 \\ 1 & 2.935420 & -1.879817 & 0.874734 \\ 1 & 0.925739 & -2.510931 & 0.856848 \\ 1 & -0.958174 & -3.358533 & 0.855913\end{array}$

$\mathrm{E}\left(\mathrm{B} 3 \mathrm{LYP} / 6-31 \mathrm{G}^{*}\right.$ opt $)=$

Sum of electronic and zero-point Energies=

Sum of electronic and thermal Energies=

Sum of electronic and thermal Enthalpies=

Sum of electronic and thermal Free Energies=
$-542.098080$

$-541.849385$

$-541.838836$

$-541.837891$

$-541.885999$

44. 11,12-Dihydro-2H[11]annulenium mono-trans planar-isoindene cation (NImag=3) 


$\begin{array}{rrrr}6 & 2.136289 & 0.255311 & 0.000005 \\ 6 & 2.971233 & -0.874090 & 0.000012 \\ 6 & 2.468589 & -2.133464 & 0.000009 \\ 6 & 1.087191 & -2.674300 & -0.000001 \\ 6 & -0.397199 & -2.106459 & -0.000012 \\ 6 & -1.344077 & -0.819951 & -0.000020 \\ 6 & -1.482540 & 0.718120 & -0.000022 \\ 6 & -0.801752 & 2.016747 & -0.000018 \\ 6 & 0.364257 & 2.782510 & -0.000010 \\ 6 & 1.768027 & 2.652733 & 0.000001 \\ 6 & 2.610615 & 1.545972 & 0.000008 \\ 1 & 4.048791 & -0.731651 & 0.000018 \\ 1 & 3.196461 & -2.945188 & 0.000015 \\ 1 & 1.106977 & -3.370386 & -0.853774 \\ 1 & -0.807484 & -2.630479 & -0.863788 \\ 1 & -1.603050 & 2.746459 & -0.000043 \\ 1 & 0.097023 & 3.836268 & -0.000021 \\ 1 & 2.275845 & 3.615454 & -0.000002 \\ 1 & 3.683624 & 1.718140 & 0.000013 \\ 1 & 1.066213 & 0.089687 & -0.000001 \\ 6 & -2.851028 & 0.965019 & 0.000028 \\ 6 & -2.618033 & -1.296698 & -0.000024 \\ 6 & -3.667514 & -0.253648 & 0.000025 \\ 1 & -4.334663 & -0.298611 & -0.875985 \\ 1 & -4.334525 & -0.298635 & 0.876149 \\ 1 & -3.291250 & 1.954829 & 0.000054 \\ 1 & -2.867776 & -2.351845 & -0.000056 \\ 1 & -0.807498 & -2.630482 & 0.863754 \\ 1 & 1.106967 & -3.370373 & 0.853783\end{array}$

E(B3LYP/6-31G* opt)

$-542.099522$

Sum of electronic and zero-point Energies $=\quad-541.850358$

Sum of electronic and thermal Energies $=\quad-541.840083$

Sum of electronic and thermal Enthalpies $=\quad-541.839139$

Sum of electronic and thermal Free Energies $=\quad-541.886077$

45. [11]Annulenium cation, di-trans, 5+4 type (26)

C, $0,-2.5235261058,-0.0282842593,-0.2345551972$

C, $0,-2.3814408767,0.0274138425,1.1711400107$

C, $0,-1.3537409925,0.0727602122,2.1412797178$

C, $0,0.0420103149,-0.0975825111,2.1166126333$

C, $0,-1.6492056471,0.1161874183,-1.3265174384$

C, $0,-0.3251997616,0.5373838647,-1.2431762761$

C, $0,0.7764071733,-0.542329195,1.0213069859$

C, $0,2.0921411485,-0.1588510546,0.7642558984$

C, $0,0.6745082615,0.1271530798,-2.1245251232$

C, $0,2.0387487391,-0.0153835583,-1.7852254207$

C, $0,2.6584367291,-0.0388051753,-0.5247981298$

H, $,-3.5421961222,-0.2762773454,-0.5290038729$

$\mathrm{H}, 0,-3.3589738839,0.0384671035,1.6519837735$

$\mathrm{H}, 0,-1.7375513865,0.3364489571,3.1256677056$

$\mathrm{H}, 0,2.7204314686,0.1448117103,1.6012110129$

$\mathrm{H}, 0,0.3892946611,-0.1807566183,-3.1301848159$

$\mathrm{H}, 0,2.7152464876,-0.1532646299,-2.6270996682$ 
$\mathrm{H}, 0,3.7406270474,0.0785232027,-0.5479741262$

$\mathrm{H}, 0,-0.0244471115,1.0614117545,-0.3461352894$

$\mathrm{H}, 0,-2.0182187619,-0.2508359668,-2.2832815604$

$\mathrm{H}, 0,0.5810165922,0.2664608764,2.9902244481$

$\mathrm{H}, 0,0.2399371133,-1.0629650277,0.2398064289$

$\mathrm{E}\left(\mathrm{B} 3 \mathrm{LYP} / 6-31 \mathrm{G}^{*}\right.$ opt $)=$

$-425.450320$

Sum of electronic and zero-point Energies=

Sum of electronic and thermal Energies=

Sum of electronic and thermal Enthalpies=

Sum of electronic and thermal Free Energies=

$-425.263902$

$-425.254711$

$-425.253766$

$\mathrm{E}\left(\mathrm{B} 3 \mathrm{LYP} / 6-311+\mathrm{G}^{* *} / / \mathrm{B} 3 \mathrm{LYP} / 6-31 \mathrm{G}^{*}\right)=$

$-425.297719$

$-425.551635$

46. [11]Annulenium cation, di-trans, $6+3$ type (27)

C, $0,1.0834105797,0.1491649411,-2.5883892265$

C, $0,1.0881785776,0.1456715943,2.1603796184$

C, $0,1.8623918044,0.0631341808,1.0035362712$

C, $0,-0.835913502,0.4664946916,-1.1218549195$

C, $0,-1.5683454116,-0.2282435025,1.9384093736$

C, $0,-2.293133691,-0.3117248164,0.7185666538$

C, $0,-2.0187836164,-0.084887745,-0.629691903$

$\mathrm{C}, 0,-0.2842676644,-0.0265603712,2.4875586882$

C, $0,-0.3046861632,0.2261306275,-2.4028027576$

C, $0,1.4032325308,-0.3883341757,-0.2337173412$

C, $0,1.9300370117,-0.002278492,-1.4804426199$

$\mathrm{H}, 0,1.5025531456,0.2068023983,-3.5896606503$

$\mathrm{H}, 0,1.6562725913,0.4747815573,3.0285923116$

$\mathrm{H}, 0,2.8563624392,0.5042997378,1.0581867628$

$\mathrm{H}, 0,-2.264929613,-0.3969457555,2.7580975836$

$\mathrm{H}, 0,-3.2954645574,-0.7009083777,0.8880058244$

$\mathrm{H}, 0,-2.737817438,-0.4885305629,-1.340784811$

$\mathrm{H}, 0,-0.3840646811,0.0328514177,3.5701901722$

$\mathrm{H}, 0,-0.9723435182,0.0397708483,-3.2429516457$

$\mathrm{H}, 0,2.9883010117,0.2380675813,-1.5734962112$

$\mathrm{H}, 0,-0.2383819221,1.0511348679,-0.4314350403$

$\mathrm{H}, 0,0.5167898088,-1.0127253079,-0.2340553209$

$\mathrm{E}\left(\mathrm{B} 3 \mathrm{LYP} / 6-31 \mathrm{G}^{*}\right.$ opt $)=$

$-425.443553$

Sum of electronic and zero-point Energies=

Sum of electronic and thermal Energies=

Sum of electronic and thermal Enthalpies=

Sum of electronic and thermal Free Energies=

$\mathrm{E}\left(\mathrm{B} 3 \mathrm{LYP} / 6-311+\mathrm{G}^{* *} / / \mathrm{B} 3 \mathrm{LYP} / 6-31 \mathrm{G}^{*}\right)=$

$-425.256954$

$-425.247839$

$-425.246895$

$-425.290844$

$-425.545249$

47. [11]Annulenium cation, tri-trans (28)

C, $0,0.985615005,0.0701616809,-2.4862932654$

C, $0,1.1446707979,0.009567901,2.4182772092$

C, $0,1.8334593031,0.2877028737,1.223546549$

C, $0,-1.1203019375,0.281590259,-1.3443990541$

C, $0,-1.0304348097,0.2480869372,1.4205403852$

C, $0,-2.1540451208,-0.3184773569,0.7666237533$

C, $0,-2.1997093289,-0.3012528664,-0.633018941$

C, $0,-0.249547926,-0.2459154887,2.4604265745$

C, $0,-0.408782118,-0.1854979148,-2.4446716699$ 
C, $0,1.3060778815,-0.0398686594,-0.0427053492$

C, $0,1.7505859494,0.3189068413,-1.3322933299$

H, $0,1.5017884645,0.0242780785,-3.4433563058$

H,0,1.7217630516,-0.0601429444,3.3383980824

H,0,2.8307394708,0.7201770245,1.2890608134

H,0,-2.8098473606,-1.0075493156,1.2938754205

H, $0,-2.888986266,-0.9779517627,-1.1329281322$

$\mathrm{H}, 0,-0.6584993973,-0.8712621463,3.2526736835$

H,0,-0.8681399349,-0.7917926336,-3.2239812012

$\mathrm{H}, 0,2.7419761222,0.7534080881,-1.4515825553$

$\mathrm{H}, 0,-0.625726526,1.1095825088,-0.8558033608$

H,0,0.4775548411,-0.7374360599,-0.0243541517

H,0,-0.5681486406,1.0886639214,0.9218005373

$\mathrm{E}\left(\mathrm{B} 3 \mathrm{LYP} / 6-31 \mathrm{G}^{*}\right.$ opt $)=$

Sum of electronic and zero-point Energies=

Sum of electronic and thermal Energies=

Sum of electronic and thermal Enthalpies=

Sum of electronic and thermal Free Energies=

$\mathrm{E}\left(\mathrm{B} 3 \mathrm{LYP} / 6-311+\mathrm{G}^{* *} / / \mathrm{B} 3 \mathrm{LYP} / 6-31 \mathrm{G}^{*}\right)=$

48. [11]Annulenium cation, mono-trans tub (29)

C,0,1.2238924405,0.0668027232,0.001723439

C, $0,1.6712464251,-0.3609857021,1.2618037959$

C,0,0.994199903,0.0142111027,2.4045642327

C, $0,-0.3008656858,0.6463741205,2.4484893004$

C, $0,-1.3748105853,0.3926402687,1.6510884824$

C, $0,-1.5636967298,-0.7020637112,0.6704866151$

C, $0,-1.5618081869,-0.7031972482,-0.6739011366$

C, $0,-1.3698832736,0.3898914496,-1.6556809207$

C, $0,-0.2936941904,0.6418779123,-2.4505669325$

C, $0,1.00107376,0.0094881237,-2.401696447$

C,0,1.6748367736,-0.3634279234,-1.2562504801

H,0,2.5617290706,-0.9794555757,1.3424726299

H,0,1.4643887516,-0.1851637495,3.3670449107

H,0,-0.4591169965,1.3315658109,3.2800380814

H,0,-2.2809847427,0.9569427981,1.8800201466

H,0,-1.8507666415,-1.6435783627,1.1433240665

H,0,-1.8475114774,-1.6454711734,-1.1460295727

$\mathrm{H}, 0,-2.275145612,0.9543327893,-1.8878439434$

H,0,-0.4493641281,1.3256437776,-3.2837787731

$\mathrm{H}, 0,1.4739960872,-0.1918584858,-3.3624244528$

H, $0,2.5655266752,-0.9820896763,-1.3330815813$

H,0,0.4943051109,0.8694651531,-0.0001012029

$\mathrm{E}(\mathrm{B} 3 \mathrm{LYP} / 6-31 \mathrm{G} *$ opt $)=$

Sum of electronic and zero-point Energies=

Sum of electronic and thermal Energies=

Sum of electronic and thermal Enthalpies=

Sum of electronic and thermal Free Energies=

$\mathrm{E}\left(\mathrm{B} 3 \mathrm{LYP} / 6-311+\mathrm{G}^{* *} / / \mathrm{B} 3 \mathrm{LYP} / 6-31 \mathrm{G}^{*}\right)=$
$-425.438692$

$-425.252429$

$-425.243595$

$-425.242651$

$-425.285584$

$-425.540516$

49. [11]Annulenium cation, mono-trans planar (NImag = 2: 52i, 95i) (30) 
C, $0,-1.0434418238,0 ., 0.7125616146$

C, $0,-0.8878389065,0 ., 2.0876203365$

C, $0,0.3676814764,0 ., 2.6895128802$

C, $0,1.6459138322,0 ., 2.1107284276$

C, $0,2.2636290135,0 ., 0.8403542393$

C, $0,2.1087409657,0 .,-0.574573913$

C, $0,1.302557947,0 .,-1.754952544$

C, $0,0.0408807802,0 .,-2.4143489638$

C, $0,-1.3670205945,0 .,-2.30117785$

C, $0,-2.3713421598,0 .,-1.321057116$

C, $0,-2.2677971445,0 ., 0.0675886165$

$\mathrm{H}, 0,-1.769085847,0 ., 2.7248445328$

$\mathrm{H}, 0,0.3757032384,0 ., 3.7766853897$

$\mathrm{H}, 0,2.4124773452,0 ., 2.8825790862$

$\mathrm{H}, 0,3.3361903853,0 ., 1.0204890415$

$\mathrm{H}, 0,3.1274452996,0 .,-0.9534242234$

$\mathrm{H}, 0,2.0262455979,0 .,-2.5658502466$

$\mathrm{H}, 0,0.2632204243,0 .,-3.4789548813$

$\mathrm{H}, 0,-1.8072030316,0 .,-3.2959508292$

$\mathrm{H}, 0,-3.3808514267,0 .,-1.7246125039$

$\mathrm{H}, 0,-3.1822077688,0 ., 0.6561811129$

$\mathrm{H}, 0,-0.1537145321,0 ., 0.1044791531$

$\mathrm{E}\left(\mathrm{B} 3 \mathrm{LYP} / 6-31 \mathrm{G}^{*}\right.$ opt $)=$

Sum of electronic and zero-point Energies=

Sum of electronic and thermal Energies=

Sum of electronic and thermal Enthalpies=

Sum of electronic and thermal Free Energies=

$\mathrm{E}\left(\mathrm{B} 3 \mathrm{LYP} / 6-311+\mathrm{G}^{* *} / / \mathrm{B} 3 \mathrm{LYP} / 6-31 \mathrm{G}^{*}\right)=$

50. [11]Annulenium cation, all cis $\mathrm{C}_{2}$ (31)

C, $0,0.6578609794,-1.0389230734,-1.7209538911$ C, $0,1.0054483939,0.1094225829,2.2832714679$

C, $0,1.3481159591,1.0488275537,1.2474813159$

C, $0,-0.9764783844,0.8271510484,-1.9707149691$

C, $0,-1.1260533836,-0.867472434,1.3278693235$

C, $0,-1.7583835166,-0.0087368233,0.4149595746$

C, $0,-1.6089794476,0.8539550979,-0.6823785224$

C, $0,0.0153966428,-0.8317998419,2.1976009115$

C, $0,-0.1204549386,-0.1049649689,-2.4921133354$

C, $0,1.4136448353,0.6955716335,-0.0559705095$

C, $0,1.2959055195,-0.6823070741,-0.5834917503$

$\mathrm{H}, 0,-1.3858023702,1.5590243921,-2.6616803101$

$\mathrm{H}, 0,-0.017206943,-0.1383714763,-3.5763928531$

$\mathrm{H}, 0,0.8131266071,-2.0378506368,-2.1232464614$

$\mathrm{H}, 0,1.8761803325,-1.4436016846,-0.0641785541$

$\mathrm{H}, 0,1.6933995101,1.4610674521,-0.7782768599$

$\mathrm{H}, 0,1.6574914037,2.0500767545,1.5400375197$

$\mathrm{H}, 0,1.5822903879,0.1462625427,3.2070305807$

$\mathrm{H}, 0,-0.0349801612,-1.5658932465,2.9970947282$

$\mathrm{H}, 0,-1.8308794433,-1.6259174327,1.6737968913$

$\mathrm{H}, 0,-2.8285493129,-0.014099204,0.6674487791$

$\mathrm{H}, 0,-2.4012059652,1.604960335,-0.6749911539$

$\mathrm{E}\left(\mathrm{B} 3 \mathrm{LYP} / 6-31 \mathrm{G}^{*}\right.$ opt $)=$
$-425.417331$

$-425.230038$

$-425.221819$

$-425.220875$

$-425.263531$

$-425.519195$ 
Sum of electronic and zero-point Energies= Sum of electronic and thermal Energies= Sum of electronic and thermal Enthalpies= Sum of electronic and thermal Free Energies= $\mathrm{E}\left(\mathrm{B} 3 \mathrm{LYP} / 6-311+\mathrm{G}^{* *} / / \mathrm{B} 3 \mathrm{LYP} / 6-31 \mathrm{G}^{*}\right)=$

51. [11]Annulenium cation, all cis $\mathrm{C}_{\mathrm{s}}$ tub (NImag=1:

C, $0,0.6336890513,-0.9068184326,-1.6775846518$ C,0,0.7641429275,0.5369309863,2.0419966696 C, $0,1.6542662984,0.9993992946,1.0250619744$ C,0,-1.1191611948,0.9795667372,-1.6257947601 C,0,-1.2300929411,-0.9669651143,1.311195433 C, $0,-2.0927153339,-0.3799174838,0.3633478346$ C,0,-2.0437570795,0.610981664,-0.6039360614 C, $0,-0.1940166623,-0.4562166213,2.1248611514$ C, $0,-0.1292213086,0.2111694509,-2.1773350181$ C,0,2.1327717995,0.2886586581,-0.0408194161

C,0,1.6313093903,-0.8686127466,-0.7465031109

$\mathrm{H}, 0,-1.4616520782,1.8051729691,-2.2443825096$ $\mathrm{H}, 0,0.0999526803,0.4406020889,-3.2209543421$ $\mathrm{H}, 0,0.6359297881,-1.7841966651,-2.3261328458$ $\mathrm{H}, 0,2.3159381113,-1.7187105619,-0.7648060785$ $\mathrm{H}, 0,3.1256432647,0.5902878844,-0.3841347808$ $\mathrm{H}, 0,2.2629026875,1.8481968926,1.3265144485$ $\mathrm{H}, 0,1.0500033284,0.9282862031,3.0197669421$

H,0,-0.2592260456,-0.8789420072,3.1291631592 $\mathrm{H}, 0,-1.6820176048,-1.8507480935,1.7601955722$ $\mathrm{H}, 0,-3.1129255707,-0.7407741555,0.5136723259$ $\mathrm{H}, 0,-3.0178382425,1.0717670908,-0.7758421585$

$\mathrm{E}\left(\mathrm{B} 3 \mathrm{LYP} / 6-31 \mathrm{G}^{*}\right.$ opt $)=$

Sum of electronic and zero-point Energies= Sum of electronic and thermal Energies= Sum of electronic and thermal Enthalpies= Sum of electronic and thermal Free Energies= $\mathrm{E}\left(\mathrm{B} 3 \mathrm{LYP} / 6-311+\mathrm{G}^{* * / / B 3 L Y P} / 6-31 \mathrm{G}^{*}\right)=$

52. [11]Trannulenium cation $\left(\mathrm{C}_{\mathrm{s}}\right)(33)$

C, $0,0.7788245753,0.3296115011,-1.8153635148$ C, $0,1.9376523543,-0.4414147952,-1.4245149011$ C,0,1.7550919244,0.3969894072,0.8791102309 C,0,1.0191373992,-0.1706591165,1.8940845562 C, $0,-0.2510927003,0.465370359,2.0907345564$ C, $0,-0.427032245,-0.2707129614,-2.0973767433$ C, $0,-1.5388088841,0.3763387956,-1.4633449936$ C,0,-2.2299195909,-0.3098045924,-0.466120358 C, $0,-2.1487993773,0.3728722574,0.7691609283$ C,0,-1.4092293664,-0.2531480414,1.7985686803 C,0,2.4073036045,-0.4090796528,-0.1283242754 $\mathrm{H}, 0,0.746283619,1.3710423641,-1.4984661945$

H,0,2.2545380451,-1.2723432061,-2.0521737451 $\mathrm{H}, 0,1.5104133028,1.4236509977,0.6105018489$ $\mathrm{H}, 0,1.137624006,-1.2252318097,2.133793659$ $\mathrm{H}, 0,-0.2819359247,1.5495512793,1.9880346948$
$-425.218457$

$-425.208432$

$-425.207488$

$-425.253715$

$-425.505277$

19i) (32)

$-425.398694$

$-425.214865$

$-425.205538$

$-425.204594$

$-425.249674$

$-425.502297$ 
$\mathrm{H}, 0,-0.472423669,-1.3366913673,-2.3101083876$

$\mathrm{H}, 0,-1.5140757092,1.4643571548,-1.4129083777$

H,0,-2.2290859189,-1.397704407,-0.469680994

$\mathrm{H}, 0,-2.1871819298,1.4614123278,0.7558388235$

$\mathrm{H}, 0,-1.3886853349,-1.3397111347,1.8487802543$

H, $0,3.065763352,-1.2165111636,0.1867034229$

$\mathrm{E}\left(\mathrm{B} 3 \mathrm{LYP} / 6-31 \mathrm{G}^{*}\right.$ opt $)=$

$-425.361515$

Sum of electronic and zero-point Energies=

Sum of electronic and thermal Energies=

$-425.176777$

$-425.167623$

Sum of electronic and thermal Enthalpies=

$-425.166678$

Sum of electronic and thermal Free Energies=

$-425.210313$

$\mathrm{E}\left(\mathrm{B} 3 \mathrm{LYP} / 6-311+\mathrm{G}^{* *} / / \mathrm{B} 3 \mathrm{LYP} / 6-31 \mathrm{G}^{*}\right)=$

$-425.464853$

53. [11]Annulenium cation, all cis $\mathrm{D}_{11 \mathrm{~h}}$ planar (NImag=2: 119.1i, 119.5i) (34)

$\mathrm{C}, 0,-2.3940550193,0 .,-0.7025568041$

C, $0,-2.3939773895,0 ., 0.703487338$

C, $0,-1.6338900935,0 ., 1.8864014973$

C, $0,-0.3548528833,0 ., 2.4703968938$

C, $0,1.036845392,0 ., 2.2698379719$

C, $0,2.0991076718,0 ., 1.3486745876$

C, $0,2.4950303717,0 .,-0.0004838096$

C, $0,2.0990008513,0 .,-1.3495960774$

C, $0,1.0363758355,0 .,-2.2703979657$

C, $0,-0.355412223,0 .,-2.4701798633$

C, $0,-1.6341896486,0 .,-1.8855887958$

$\mathrm{H}, 0,-3.4379138944,0 .,-1.0088866066$

$\mathrm{H}, 0,-3.4377985689,0 ., 1.0100010975$

$\mathrm{H}, 0,-2.3463093379,0 ., 2.7085646681$

$\mathrm{H}, 0,-0.5093888114,0 ., 3.5472575294$

$\mathrm{H}, 0,1.4890948544,0 ., 3.2592609732$

$\mathrm{H}, 0,3.0144761718,0 ., 1.9365281291$

$\mathrm{H}, 0,3.582905048,0 .,-0.0004676773$

$\mathrm{H}, 0,3.0141983774,0 .,-1.9377295361$

$\mathrm{H}, 0,1.488261349,0 .,-3.2599987346$

$\mathrm{H}, 0,-0.5105746955,0 .,-3.5469426178$

$\mathrm{H}, 0,-2.346847683,0 .,-2.7075570597$

$\mathrm{E}\left(\mathrm{B} 3 \mathrm{LYP} / 6-31 \mathrm{G}^{*}\right.$ opt $)=$

$-425.351668$

Sum of electronic and zero-point Energies $=\quad-425.163862$

Sum of electronic and thermal Energies $=\quad-425.155236$

Sum of electronic and thermal Enthalpies $=\quad-425.154291$

Sum of electronic and thermal Free Energies $=\quad-425.199499$

$\mathrm{E}\left(\mathrm{B} 3 \mathrm{LYP} / 6-311+\mathrm{G}^{* *} / / \mathrm{B} 3 \mathrm{LYP} / 6-31 \mathrm{G}^{*}\right)=\quad-425.454155$

54. [11]Annulenium cation triplet, di-trans 5+4 type (26t)

$\mathrm{C}, 0,-2.5041877539,-0.0574444964,-0.4618404105$

C, $0,-2.5600058712,-0.0005029615,0.9532750316$

C, $0,-1.5922420828,0.0566394801,1.9872362289$

$\mathrm{C}, 0,-0.2162045911,-0.1327138213,1.970247934$

C, $0,-1.4522397174,0.1323904329,-1.3489638688$

C, $, 0,-0.1390365161,0.6067035557,-1.0873694974$

C, $0,0.6062400342,-0.6066109714,0.9134056413$

C, $0,2.0058499319,-0.2448340525,0.8867616075$ 
C, $0,0.9372499014,0.2454247565,-1.9826908981$

C, $0,2.2235944561,-0.0054708601,-1.6056164317$

C, $0,2.7321927623,0.006419673,-0.2397902421$

$\mathrm{H}, 0,-3.4539166433,-0.3115788723,-0.926677563$

$\mathrm{H}, 0,-3.5808304151,-0.0006960027,1.3334014331$

$\mathrm{H}, 0,-2.0067234377,0.3105088418,2.9600660569$

$\mathrm{H}, 0,2.4850170171,-0.0712312889,1.8499307806$

$\mathrm{H}, 0,0.6698115616,0.0719132928,-3.0247104581$

$\mathrm{H}, 0,2.9418386769,-0.2488474913,-2.3850958584$

$\mathrm{H}, 0,3.7852456185,0.2501433817,-0.1199000577$

$\mathrm{H}, 0,0.036106605,1.3167584917,-0.2863916767$

$\mathrm{H}, 0,-1.6477947606,-0.1712003248,-2.3781195452$

$\mathrm{H}, 0,0.3088827781,0.1709482325,2.8766931459$

$\mathrm{H}, 0,0.2150996799,-1.3167226699,0.1928731744$

$\mathrm{E}\left(\mathrm{UB} 3 \mathrm{LYP} / 6-31 \mathrm{G}^{*}\right.$ opt $)=$

$-425.397386$

Sum of electronic and zero-point Energies=

$-425.214827$

Sum of electronic and thermal Energies=

$-425.205132$

Sum of electronic and thermal Enthalpies=

$-425.204187$

Sum of electronic and thermal Free Energies=

$-425.250250$

$\mathrm{E}\left(\mathrm{UB} 3 \mathrm{LYP} / 6-311+\mathrm{G}^{* *} / / \mathrm{UB} 3 \mathrm{LYP} / 6-31 \mathrm{G}^{*}\right)=$

$-425.499519$

55. [11]Annulenium cation triplet, di-trans $6+3$ type $(\mathbf{2 7 t})$

C, $0,1.0217359469,0.2520600719,-2.4844072179$

C, $0,1.0588645575,0.376997802,2.1557345277$

C, $0,1.9178495675,0.018080984,1.0975694919$

C, $0,-0.9251810235,0.515854266,-1.0306500072$

C, $0,-1.3009155329,-0.695215246,1.7355933213$

C, $0,-2.2318832614,-0.6663797283,0.6965272295$

C, $0,-2.1319451574,-0.0709022473,-0.5769788866$

C, $0,-0.3317697995,0.2924399846,2.2343645036$

C, $0,-0.373617823,0.3447042424,-2.3055108469$

C, $0,1.4150943522,-0.3949949387,-0.1606001985$

C, $0,1.8986847774,0.0315285448,-1.4027766059$

$\mathrm{H}, 0,1.4337247671,0.3536996967,-3.4861770295$

$\mathrm{H}, 0,1.5196739446,1.0120756676,2.9100962557$

$\mathrm{H}, 0,2.9420687144,0.3799952609,1.1555328101$

$\mathrm{H}, 0,-1.3880171658,-1.5583228557,2.4021553886$

$\mathrm{H}, 0,-3.0416773892,-1.3875515694,0.7907362274$

$\mathrm{H}, 0,-2.8772226892,-0.3640129533,-1.3131946371$

$\mathrm{H}, 0,-0.7955701318,1.0196501719,2.9073433279$

$\mathrm{H}, 0,-1.0257143168,0.171458044,-3.1603211371$

$\mathrm{H}, 0,2.9476314269,0.3026782526,-1.5129295619$

$\mathrm{H}, 0,-0.31313817,1.0191255566,-0.2886521815$

H, $0,0.4967413879,-0.9738376833,-0.1577813283$

$\mathrm{E}\left(\mathrm{UB} 3 \mathrm{LYP} / 6-31 \mathrm{G}^{*}\right.$ opt $)=$

$-425.375364$

Sum of electronic and zero-point Energies=

$-425.193591$

Sum of electronic and thermal Energies=

Sum of electronic and thermal Enthalpies=

$-425.183961$

$-425.183017$

$-425.228825$

$\mathrm{E}\left(\mathrm{UB} 3 \mathrm{LYP} / 6-311+\mathrm{G}^{* *} / / \mathrm{UB} 3 \mathrm{LYP} / 6-31 \mathrm{G}^{*}\right)=$

$-425.477511$

56. [11]Annulenium cation triplet, tri-trans (28t) 
C, $0,2.4982925512,-1.083297735,0.0076585809$

C, $0,-2.4045918587,-1.009031481,-0.2083688796$

C, $0,-1.2109552254,-1.7975352978,-0.2299865681$

C, $0,1.3314544448,0.993603418,0.4504262775$

C, $0,-1.5162493436,1.2598106647,0.587435463$

C, $0,-0.6613961501,2.0806916782,-0.2324631093$

C, $0,0.7178123071,2.1293059653,-0.1472400653$

C, $0,-2.5528854944,0.314165705,0.1605986982$

C, $0,2.4977955391,0.3212783269,0.0640320441$

C, $0,0.0766830879,-1.2705147672,-0.3880665231$

C, $0,1.3088618156,-1.8494338344,-0.051028747$

$\mathrm{H}, 0,3.4502615041,-1.6060596118,-0.0592395211$

$\mathrm{H}, 0,-3.3336378409,-1.5652292085,-0.3102599342$

$\mathrm{H}, 0,-1.3319092872,-2.8626934678,-0.0370022451$

$\mathrm{H}, 0,-1.1317463863,2.6153730127,-1.059851184$

$\mathrm{H}, 0,1.2791040342,2.7829315615,-0.8087208556$

$\mathrm{H}, 0,-3.5814684816,0.6310995126,0.351607454$

$\mathrm{H}, 0,3.3717078139,0.8689869476,-0.2861486454$

$\mathrm{H}, 0,1.3657725987,-2.9152071358,0.1639915037$

$\mathrm{H}, 0,0.6965542339,0.4439531275,1.1415389943$

$\mathrm{H}, 0,0.1193800244,-0.2934636711,-0.8456778437$

$\mathrm{H}, 0,-1.412948254,1.3660530773,1.6717792492$

$\mathrm{E}\left(\mathrm{UB} 3 \mathrm{LYP} / 6-31 \mathrm{G}^{*}\right.$ opt $)=$

Sum of electronic and zero-point Energies=

Sum of electronic and thermal Energies=

Sum of electronic and thermal Enthalpies=

Sum of electronic and thermal Free Energies=

$\mathrm{E}\left(\mathrm{UB} 3 \mathrm{LYP} / 6-311+\mathrm{G}^{* *} / / \mathrm{UB} 3 \mathrm{LYP} / 6-31 \mathrm{G}^{*}\right)=$

57. [11]Annulenium cation triplet, mono-trans (29t)

C, $0,1.293017384,-0.1905758638,0.1061215815$

C, $0,1.6858469733,0.1526269613,-1.1853882388$

C, $0,0.9563603384,-0.2807092255,-2.2997513816$

C, $0,-0.4219557547,-0.5954548437,-2.4386870783$

C, $0,-1.590190032,-0.0934140975,-1.8231383809$

C, $0,-1.8396181626,0.7331732684,-0.7057756711$

C, $0,-1.322098177,0.7094965141,0.6076083637$

C, $0,-1.147217234,-0.4192850204,1.4132526853$

C, $0,-0.2858447778,-0.4512395314,2.5587093727$

C, $0,1.0075305357,0.0606919436,2.5366404669$

C, $0,1.7197822478,0.330110102,1.3394841325$

$\mathrm{H}, 0,2.5959952982,0.7267828382,-1.3522873767$

$\mathrm{H}, 0,1.4982475104,-0.3066774984,-3.2451762578$

$\mathrm{H}, 0,-0.6415718932,-1.0884792826,-3.3833908512$

$\mathrm{H}, 0,-2.4456977733,-0.154126412,-2.4986086416$

$\mathrm{H}, 0,-2.5485221265,1.540402599,-0.9106728473$

$\mathrm{H}, 0,-1.1576650493,1.678356774,1.0825921322$

$\mathrm{H}, 0,-1.7180798071,-1.3175767745,1.1739030857$

H, $0,-0.6333702842,-0.9547336899,3.4601250023$

$\mathrm{H}, 0,1.5283025819,0.2001796777,3.4815899716$

$\mathrm{H}, 0,2.6250031335,0.9322211327,1.3895683196$

H, $0,0.5636783626,-0.9888706068,0.1479023526$

$\mathrm{E}\left(\mathrm{UB} 3 \mathrm{LYP} / 6-31 \mathrm{G}^{*}\right.$ opt $)=$
$-425.371084$

$-425.189788$

$-425.179944$

$-425.179000$

$-425.225245$

$-425.473074$ 
Sum of electronic and zero-point Energies= Sum of electronic and thermal Energies= Sum of electronic and thermal Enthalpies= Sum of electronic and thermal Free Energies= $\mathrm{E}\left(\mathrm{UB} 3 \mathrm{LYP} / 6-311+\mathrm{G}^{* *} / / \mathrm{UB} 3 \mathrm{LYP} / 6-31 \mathrm{G}^{*}\right)=$

58. [11]Annulenium cation triplet, all cis (31t)

C, $0,-1.1874292534,1.0991561316,-1.5180426779$ C, $0,-0.4627445581,-0.343275692,2.4301619642$

C, $0,-1.1875067981,-1.0991343223,1.5179923362$

C,0,0.5123989574,-0.6455919027,-2.1407561126

C, $0,1.466207148,0.7682343576,1.1087374031$

C,0,1.9020063431,0.0000206918,0.0000195911

C, $0,1.4662167669,-0.7682492158,-1.1086753939$

C, $0,0.5123606647,0.6455494951,2.1407946178$

C,0,-0.4626706824,0.3432534266,-2.430180707

C,0,-1.3771105425,-0.6763729452,0.1932095442

C,0,-1.3770838631,0.6764128647,-0.1932628986

$\mathrm{H}, 0,0.717291799,-1.31680971,-2.9727744651$

H,0,-0.6089337986,0.5493545899,-3.4900957853

$\mathrm{H}, 0,-1.6314070243,2.0377197651,-1.8433737727$

$\mathrm{H}, 0,-1.620804259,1.4216774903,0.5610391906$

$\mathrm{H}, 0,-1.6208366479,-1.4216269969,-0.561101278$

$\mathrm{H}, 0,-1.6315326911,-2.0376830729,1.8433006462$

H,0,-0.6090414305,-0.5494002523,3.4900679321

H,0,0.7172529674,1.3167274967,2.9728452166

H, $0,2.2313781197,1.4948017892,1.3832383603$

H,0,2.9973769259,0.000053869,-0.000002709

H,0,2.2313909449,-1.4948323038,-1.3831293356

$\mathrm{E}(\mathrm{UB} 3 \mathrm{LYP} / 6-31 \mathrm{G} *$ opt $)=$

Sum of electronic and zero-point Energies= Sum of electronic and thermal Energies= Sum of electronic and thermal Enthalpies= Sum of electronic and thermal Free Energies= $\mathrm{E}\left(\mathrm{UB} 3 \mathrm{LYP} / 6-311+\mathrm{G}^{* *} / / \mathrm{UB} 3 \mathrm{LYP} / 6-31 \mathrm{G}^{*}\right)=$

59. [11]Trannulenium cation triplet $\left(\mathrm{C}_{2}\right)(\mathbf{3 3 t})$

C,0,0.9987757731,0.2796443035,-2.1709590896

C,0,2.2107045487,-0.1708666621,-1.6131243657

C, $0,1.47098396,0.0678550957,0.714732595$

C, $0,0.8486728343,-0.4467062221,1.8424343217$

$\mathrm{C}, 0,-0.3306484412,0.3160706302,2.1897445511$

C, $0,-0.2434792157,-0.1628228382,-1.610013101$

C, $0,-1.448311487,0.4928920072,-1.4049615912$

C, $0,-2.2086619868,-0.1197538024,-0.3372263043$

C,0,-2.2506028604,0.5314361637,0.8940025176

C, $0,-1.5769609895,-0.2352553551,1.8989197244$

C,0,2.3312104161,-0.5371352384,-0.2587306496

$\mathrm{H}, 0,1.007858682,0.9061428141,-3.061457937$

H,0,3.0891148159,-0.2387103245,-2.2542037123

$\mathrm{H}, 0,1.1378251247,1.0620588084,0.4214855604$

$\mathrm{H}, 0,0.9734680471,-1.4811521771,2.153532076$

$\mathrm{H}, 0,-0.2374881527,1.3988760294,2.1163311211$
$-425.208664$

$-425.198866$

$-425.197922$

$-425.244142$

$-425.492830$

$-425.388301$

$-425.205557$

$-425.195773$

$-425.194828$

$-425.241484$

$-425.490696$ 
$\mathrm{H}, 0,-0.1650984878,-1.1371575534,-1.1304836482$

$\mathrm{H}, 0,-1.6021786766,1.5293890947,-1.6955381889$

$\mathrm{H}, 0,-2.220213567,-1.2088892583,-0.3234622881$

$\mathrm{H}, 0,-2.2006164831,1.6180360935,0.932770948$

$\mathrm{H}, 0,-1.708367554,-1.3155325911,1.9196317041$

$\mathrm{H}, 0,3.1156009422,-1.2252094281,0.0524827148$

$\mathrm{E}(\mathrm{UB} 3 \mathrm{LYP} / 6-31 \mathrm{G} *$ opt $)=$

$-425.326441$

Sum of electronic and zero-point Energies= $-425.145198$

Sum of electronic and thermal Energies=

$-425.135318$

Sum of electronic and thermal Enthalpies=

$-425.134374$

Sum of electronic and thermal Free Energies=

$-425.180502$

$\mathrm{E}\left(\mathrm{UB} 3 \mathrm{LYP} / 6-311+\mathrm{G}^{* *} / / \mathrm{UB} 3 \mathrm{LYP} / 6-31 \mathrm{G}^{*}\right)=$

$-425.429178$

60. [11]Annulenium, di-trans, 5+4 type-indene cation (35)

C, $0,-3.3485563994,-0.0719993488,-0.1419012597$

C, $0,-3.3454742408,-0.0674822889,1.2604661834$

C, $0,-2.4132919343,-0.0135700443,2.3395052133$

C, $0,-1.0315872872,-0.1550900294,2.4611117007$

C, $0,-2.3577533121,0.0973967963,-1.1411900665$

C, $0,-1.0730523158,0.5567993159,-0.924311355$

$\mathrm{C}, 0,-0.1668043,-0.5220755274,1.4181746585$

C, $0,1.1518366432,-0.1224612962,1.3068646567$

C, $0,0.0314161633,0.2306777213,-1.730746699$

C, $0,1.3401795475,0.0672199476,-1.2680919027$

C, $0,1.8548532057,-0.0103030794,0.0720717063$

$\mathrm{H}, 0,-4.3317450416,-0.3035013365,-0.5487076239$

$\mathrm{H}, 0,-4.3630655777,-0.1096223417,1.6470684514$

$\mathrm{H}, 0,-2.9075127894,0.2222597964,3.2808496205$

$\mathrm{H}, 0,1.6856569,0.19676233,2.2019377468$

$\mathrm{H}, 0,-0.1449663758,0.0198554667,-2.7864101441$

$\mathrm{H}, 0,-0.8731379855,1.0530595673,0.0152497593$

$\mathrm{H}, 0,-2.6180623107,-0.2755370559,-2.13140567$

$\mathrm{H}, 0,-0.5962629124,0.1623423818,3.4073601449$

$\mathrm{H}, 0,-0.612442081,-1.0251559234,0.571373483$

C, $0,3.3042488031,0.0417539413,-0.002707182$

C, $0,2.5234906628,-0.0176998166,-2.2102446433$

C, $0,3.7052619905,0.0163478247,-1.2954534271$

$\mathrm{H}, 0,3.9492554634,0.0356650713,0.8686850347$

$\mathrm{H}, 0,2.5014603592,-0.9480241616,-2.8001886296$

$\mathrm{H}, 0,4.7316164637,-0.0064264948,-1.6435055272$

$\mathrm{H}, 0,2.5306025291,0.8012380047,-2.9435921467$

$\mathrm{E}\left(\mathrm{B} 3 \mathrm{LYP} / 6-31 \mathrm{G}^{*} \mathrm{opt}\right)=$

$-540.981486$

Sum of electronic and zero-point Energies=

Sum of electronic and thermal Energies=

$-540.755060$

$-540.743750$

$-540.742806$

Sum of electronic and thermal Enthalpies=

$-540.791676$

Sum of electronic and thermal Free Energies=

$-541.108852$

$\chi=-124.5$

61. [11]Annulenium, di-trans, 5+4 type-isoindene cation (36)

C, $0,-3.3457601081,-0.0922308152,-0.1811247654$

C, $, 0,-3.3530929244,-0.0589490638,1.2355087696$ 
C,0,-2.4293760492,-0.0085983359,2.3093035582

C, $0,-1.0514089431,-0.1814448929,2.40665008$

C, $0,-2.3659869486,0.1219301249,-1.1470522916$

C, $0,-1.0471388138,0.5912247062,-0.9305742074$

C, $0,-0.1739455943,-0.6120497988,1.3810165856$

C, $0,1.1145747545,-0.1407370483,1.3197486483$

C, $0,-0.0127892827,0.161315649,-1.7255469136$

C, $0,1.3679201476,0.0647985034,-1.3044122713$

C, $0,1.8886453333,-0.0075633382,0.1045311942$

$\mathrm{H}, 0,-4.3189442069,-0.3540737669,-0.5918475622$

$\mathrm{H}, 0,-4.3757575758,-0.077425332,1.6124596563$

$\mathrm{H}, 0,-2.9113368535,0.2279650967,3.255933649$

$\mathrm{H}, 0,1.5690615846,0.2540906143,2.2290923855$

$\mathrm{H}, 0,-0.24480578,-0.2281307913,-2.717442766$

$\mathrm{H}, 0,-0.8358049493,1.1132419618,-0.0090903975$

$\mathrm{H}, 0,-2.6234836773,-0.1859684999,-2.1605594769$

$\mathrm{H}, 0,-0.5994541738,0.1294601868,3.348648518$

$\mathrm{H}, 0,-0.5946227549,-1.1384328444,0.5370336282$

C,0,3.2514832607,0.0389290712,0.0549396208

C, $0,2.4375706682,0.0594949715,-2.1517817453$

C, $0,3.7092708644,0.0639566034,-1.3672885428$

$\mathrm{H}, 0,3.9208256066,0.0016183393,0.9069329769$

$\mathrm{H}, 0,4.3526919924,-0.7977813241,-1.6101284129$

$\mathrm{H}, 0,2.3922747768,0.1072799177,-3.2338979466$

$\mathrm{H}, 0,4.329557824,0.9476984231,-1.5906405672$

$\mathrm{E}\left(\mathrm{B} 3 \mathrm{LYP} / 6-31 \mathrm{G}^{*}\right.$ opt $)=$

$-540.960361$

Sum of electronic and zero-point Energies=

Sum of electronic and thermal Energies=

$-540.734806$

$-540.723386$

$-540.722442$

Sum of electronic and thermal Enthalpies=

$\begin{array}{ll}\text { Sum of electronic and thermal Free Energies }= & -540.771508 \\ \text { E(B3LYP/6-311+G**//B3LYP/6-31G*)= } & -541.088368\end{array}$

$\chi=-82.1$

62. [11]Annulenium, di-trans, $6+3$ type-indene cation (37)

C, $0,1.3636841492,0.4405209823,-3.4259240033$

C, $0,1.3815709032,0.3223913418,1.3289288184$

C, $0,2.1418504941,0.3790120835,0.1520906866$

C, $0,-0.5626682106,0.4833524154,-1.9376882665$

C, $0,-1.2261670125,-0.3713809791,1.162799132$

C, $0,-1.8860444996,-0.547052734,-0.1025342319$

C, $0,-1.6524362619,-0.2420235009,-1.4320322165$

C, $0,0.0616925145,-0.0027398552,1.7122486925$

C, $0,-0.0274098053,0.3166141875,-3.2184970975$

C, $0,1.7554777938,-0.1063700823,-1.0864451941$

C, $0,2.2319936588,0.3544148927,-2.3391117816$

$\mathrm{H}, 0,1.7554550772,0.5864259428,-4.4293153636$

$\mathrm{H}, 0,1.9488369519,0.6875066239,2.1848026116$

$\mathrm{H}, 0,3.0565139375,0.9665448586,0.2147252621$

$\mathrm{H}, 0,-2.8160208157,-1.0960322005,0.0302798624$

$\mathrm{H}, 0,-2.3175386058,-0.7207705167,-2.1493711093$

$\mathrm{H}, 0,-0.669821115,0.0109220432,-4.0436356959$

$\mathrm{H}, 0,3.2624932068,0.6921170676,-2.4382410218$

$\mathrm{H}, 0,-0.0375502109,1.134410882,-1.2486453861$

$\mathrm{H}, 0,0.956608305,-0.8392603239,-1.1011683692$ 
C, $0,-2.1044200119,-0.6617223498,2.302300758$

C, $0,-0.1095894386,0.0206174629,3.2344442634$

C, $0,-1.4958912705,-0.43722536,3.4814507526$

$\mathrm{H}, 0,0.6395727793,-0.6121277933,3.7328956358$

$\mathrm{H}, 0,0.0569953763,1.0390989295,3.6194552028$

$\mathrm{H}, 0,-3.1309609448,-0.9851549814,2.170664035$

$\mathrm{H}, 0,-1.9344419584,-0.5541315608,4.4653724633$

$\mathrm{E}\left(\mathrm{B} 3 \mathrm{LYP} / 6-31 \mathrm{G}^{*}\right.$ opt $)=$

$-540.969695$

Sum of electronic and zero-point Energies=

Sum of electronic and thermal Energies=

$-540.743372$

$-540.732188$

Sum of electronic and thermal Enthalpies=

$-540.731244$

Sum of electronic and thermal Free Energies=

$-540.780059$

$\mathrm{E}\left(\mathrm{B} 3 \mathrm{LYP} / 6-311+\mathrm{G}^{* *} / / \mathrm{B} 3 \mathrm{LYP} / 6-31 \mathrm{G}^{*}\right)=$

$-541.097158$

$\chi=-123.2$

63. [11]Annulenium, di-trans, $6+3$ type-isoindene cation (38)

C, $0,1.3765000677,0.4554749874,-3.4282437939$

C, $0,1.4353937614,0.3649777811,1.3363284975$

C, $0,2.1752542604,0.362472809,0.184141867$

C, $0,-0.5313505594,0.4805378779,-1.9326532938$

C, $0,-1.2644421525,-0.4005723138,1.1701779432$

C, $0,-1.9207808414,-0.5236837441,-0.1290830011$

$\mathrm{C}, 0,-1.6731435662,-0.1951317759,-1.4351555279$

C, $0,0.0850493894,0.0104824732,1.7665981067$

C, $0,-0.006982491,0.3440725273,-3.2247208133$

C, $0,1.7460799408,-0.0781837499,-1.0924396066$

C, $0,2.2328616064,0.3927599384,-2.3188356241$

$\mathrm{H}, 0,1.7786658294,0.588348598,-4.429774056$

$\mathrm{H}, 0,1.9876596448,0.7785482968,2.1776899282$

$\mathrm{H}, 0,3.13503216,0.8731846148,0.2340070255$

$\mathrm{H}, 0,-2.8583575258,-1.0633829073,-0.01246764$

$\mathrm{H}, 0,-2.3584022757,-0.61460334,-2.1692935894$

$\mathrm{H}, 0,-0.6573507787,0.053753054,-4.0480605387$

$\mathrm{H}, 0,3.2397578487,0.8015304576,-2.3838853859$

$\mathrm{H}, 0,-0.0211689764,1.1570377134,-1.2538725378$

$\mathrm{H}, 0,0.9744097114,-0.8414100919,-1.119529616$

C, $0,-2.0837479064,-0.7004303749,2.2294356197$

C, $, 0,-0.067759033,-0.01348895,3.1300802834$

C, $0,-1.417840174,-0.4709342874,3.532370606$

$\mathrm{H}, 0,-3.1104370191,-1.0347526351,2.1356689573$

$\mathrm{H}, 0,-1.3843299131,-1.3809558362,4.1536524818$

$\mathrm{H}, 0,-1.9479327589,0.2739313727,4.1483996934$

$\mathrm{H}, 0,0.71190024,0.2386515136,3.8394577006$

$\mathrm{E}\left(\mathrm{B} 3 \mathrm{LYP} / 6-31 \mathrm{G}^{*}\right.$ opt $)=$

$-540.948263$

$-540.722833$

$-540.711503$

$-540.710559$

$-540.759799$

Sum of electronic and thermal Free Energies=

$-541.076447$

$\chi=-79.7$

64. [11]Annulenium, tri-trans-indene cation (39) 
C, $0,1.835747365,-0.2007816378,-2.441955573$

C, $0,1.8224039675,-0.2301057786,2.4648391488$ C, $0,2.6022064995,-0.2047625462,1.3068164525$ C, $0,-0.158418563,0.6185857756,-1.3734303944$ C, $0,-0.1699792416,0.6526313195,1.4234724252$ C,0,-1.3975349927,0.4627285777,0.7206803504 C, $0,-1.3818539494,0.4570405907,-0.7115520885$ C,0,0.4011595531,-0.0807619779,2.4463195666 C, $0,0.4457521274,0.0017223118,-2.4805324407$ C, $0,2.0443528383,-0.3745981133,0.0114988971$ C,0,2.6078773303,-0.1711004413,-1.2499164694 $\mathrm{H}, 0,2.3536969295,-0.4281853131,-3.37191324$ $\mathrm{H}, 0,2.3173970214,-0.4246794225,3.4144572024$ $\mathrm{H}, 0,3.6823740633,-0.1118854622,1.4095677261$ H,0,-0.1953869984,-0.6036290845,3.1928987107 H,0,-0.1236444963,-0.3645729138,-3.3324050499 $\mathrm{H}, 0,3.6826244722,-0.0196149211,-1.3407141271$ $\mathrm{H}, 0,0.555838834,1.2173347165,-0.8264094555$ $\mathrm{H}, 0,1.0405342164,-0.7809970294,0.0070438749$ $\mathrm{H}, 0,0.5316420665,1.3214667825,0.9419718649$ C, $0,-2.6600304267,-0.098548723,1.1692512566$ C, $0,-2.6851695015,-0.139592865,-1.1847492427$ C, $0,-3.4241578635,-0.4050873831,0.0957037413$ $\mathrm{H}, 0,-2.9379084238,-0.2152857209,2.2101351493$ $\mathrm{H}, 0,-2.5267758176,-1.0660699834,-1.7550614293$ $\mathrm{H}, 0,-4.4387647778,-0.7854053708,0.1261184125$ $\mathrm{H}, 0,-3.2357579459,0.5373090686,-1.8543634181$

$\mathrm{E}\left(\mathrm{B} 3 \mathrm{LYP} / 6-31 \mathrm{G}^{*}\right.$ opt $)=$ Sum of electronic and zero-point Energies= Sum of electronic and thermal Energies= Sum of electronic and thermal Enthalpies= Sum of electronic and thermal Free Energies= $\mathrm{E}\left(\mathrm{B} 3 \mathrm{LYP} / 6-311+\mathrm{G}^{* *} / / \mathrm{B} 3 \mathrm{LYP} / 6-31 \mathrm{G}^{*}\right)=$ $\chi=-120.4$

65. [11]Annulenium, tri-trans-isoindene cation (40)

C,0,1.9157980766,-0.1487798844,-2.4368786866 C,0,1.9115599237,-0.1498990259,2.4401347373 C,0,2.675049828,0.0102572843,1.2844996806 C, $0,-0.1792707772,0.4932332423,-1.4268761838$ C, $0,-0.1817380124,0.4925276444,1.4267562322$ $\mathrm{C}, 0,-1.4512430331,0.2758022548,0.7601814508$ C, $0,-1.4499533782,0.2762233107,-0.7625972592$ C, $0,0.4793298084,-0.2165314428,2.3949466849$ C, $0,0.483472939,-0.2154703827,-2.394159603$ C, $0,2.1288898481,-0.2296318467,0.0017937191$ C,0,2.6772847358,0.0108319917,-1.2798699913 $\mathrm{H}, 0,2.4308962035,-0.2669765057,-3.3889253185$ $\mathrm{H}, 0,2.4250277162,-0.2686038807,3.3929979543$ $\mathrm{H}, 0,3.7280649697,0.2692929125,1.3762344881$ $\mathrm{H}, 0,-0.043976411,-0.8788656711,3.0834492727$ $\mathrm{H}, 0,-0.0386289094,-0.8775958282,-3.0837780574$ $\mathrm{H}, 0,3.7304616162,0.2698918287,-1.369656257$ $\mathrm{H}, 0,0.4232520333,1.2755412246,-0.9745561138$
$-540.966250$ $-540.739738$

$-540.728723$

$-540.727779$

$-540.775817$

$-541.093914$ 
H,0,1.200537261,-0.7885697584,0.0008554886

$\mathrm{H}, 0,0.4215734718,1.2750120675,0.9758029121$

C, $0,-2.6924177709,-0.0667669367,1.1818284724$

C,0,-2.6904192704,-0.0660553242,-1.1865300101

C,0,-3.5861947837,-0.2768087775,-0.0031651013

$\mathrm{H}, 0,-4.4416649859,0.4168867878,-0.0036732098$

H,0,-4.0349628966,-1.2834236263,-0.0038380616

H,0,-3.022607308,-0.1387230209,2.2113214252

H,0,-3.0188615646,-0.1374591739,-2.2166193756

$\mathrm{E}\left(\mathrm{B} 3 \mathrm{LYP} / 6-31 \mathrm{G}^{*}\right.$ opt $)=$

Sum of electronic and zero-point Energies=

Sum of electronic and thermal Energies=

Sum of electronic and thermal Enthalpies=

Sum of electronic and thermal Free Energies= $\mathrm{E}\left(\mathrm{B} 3 \mathrm{LYP} / 6-311+\mathrm{G}^{* *} / / \mathrm{B} 3 \mathrm{LYP} / 6-31 \mathrm{G}^{*}\right)=$

$\chi=-92.9$
$-540.946203$

$-540.721272$

$-540.709949$

$-540.709005$

$-540.757775$

$-541.074172$

66. [11]Annulenium, mono-trans tub-indene cation (41)

C, $0,-0.9597517998,-1.4489705555,0.3900017961$

C, $0,-0.9846930466,-1.5128213439,1.7953397826$

C, $0,0.1924716866,-1.4831714355,2.5044788357$

C, $0,1.4858068995,-1.1673942719,1.9563301731$

C,0,1.791831069,-0.252249738,0.9950747131

C,0,0.9709660308,0.7908341078,0.3455182688

C, $0,0.3786439303,0.8942693106,-0.9016852736$

C,0,0.2740951562,0.0366977519,-2.079183972

C, $0,-0.6627883095,-0.8785498496,-2.4921440794$

C, $0,-1.8626477283,-1.2959405197,-1.8351290065$

C,0,-2.0588284825,-1.4090407422,-0.4721321199

$\mathrm{H}, 0,-1.9316329103,-1.6273033882,2.3167091985$

H,0,0.1643260134,-1.7082629536,3.5693851264

H,0,2.3328280942,-1.6343042918,2.4554979537

$\mathrm{H}, 0,2.8593644563,-0.100145564,0.8250990487$

$\mathrm{H}, 0,0.9541158394,0.3453768167,-2.8770589654$

$\mathrm{H}, 0,-0.5791129234,-1.1827911727,-3.5331055693$

H,0,-2.7073728909,-1.4982560892,-2.4921784947

$\mathrm{H}, 0,-3.0660001814,-1.5229698574,-0.078576291$

$\mathrm{H}, 0,0.0079897903,-1.5840457292,-0.0782020715$

C, $0,0.0685584609,2.370590479,-1.1420343337$

C, $0,1.0100513142,2.1268028693,0.9633723048$

C,0,0.4571013459,3.0358420539,0.1366962147

$\mathrm{H}, 0,-0.9842821844,2.5347837518,-1.4126813432$

$\mathrm{H}, 0,1.4089997257,2.3050881795,1.9549889067$

$\mathrm{H}, 0,0.2766952569,4.0824193042,0.3526937541$

H,0,0.6591827544,2.7490222971,-1.9895910758

$\mathrm{E}\left(\mathrm{B} 3 \mathrm{LYP} / 6-31 \mathrm{G}^{*}\right.$ opt $)=$

$-540.958567$

Sum of electronic and zero-point Energies=

$-540.733247$

Sum of electronic and thermal Energies=

$-540.721677$

Sum of electronic and thermal Enthalpies=

$-540.720733$

Sum of electronic and thermal Free Energies=

$-540.770535$

$\mathrm{E}\left(\mathrm{B} 3 \mathrm{LYP} / 6-311+\mathrm{G}^{* *} / / \mathrm{B} 3 \mathrm{LYP} / 6-31 \mathrm{G}^{*}\right)=$

$-541.087320$ 
67. [11]Annulenium, mono-trans tub-isoindene cation (42)

C, $0,-0.9654945564,-1.3810951131,0.3623844473$

C, $0,-0.9600066836,-1.3661305422,1.7646654184$

C, $0,0.2322198859,-1.3694348011,2.4675752498$

C, $0,1.5433997201,-1.1865275595,1.8996487787$

C, $0,1.9106296329,-0.333317925,0.9011239486$

C, $0,1.1255587117,0.7877814107,0.3528386365$

C, $0,0.4691950746,0.891650882,-0.9977376067$

$\mathrm{C}, 0,0.4597126474,-0.1033768654,-2.0856075591$

C, $0,-0.6007982811,-0.8459385702,-2.5143803563$

$\mathrm{C}, 0,-1.86907055,-1.0354981479,-1.8581685741$

C, $0,-2.0581310129,-1.1916232683,-0.4959951867$

$\mathrm{H}, 0,-1.9002992206,-1.3680761347,2.3108075773$

H,0,0.1882606977,-1.5541650077,3.5406084864

$\mathrm{H}, 0,2.3364481073,-1.7706624436,2.366771487$

$\mathrm{H}, 0,2.9420468856,-0.4155107052,0.5528927894$

$\mathrm{H}, 0,1.3671117056,-0.1665275276,-2.6893354851$

$\mathrm{H}, 0,-0.5097052674,-1.3185734844,-3.4924744966$

$\mathrm{H}, 0,-2.7468995122,-1.0878709021,-2.5016961193$

$\mathrm{H}, 0,-3.0684593925,-1.1824528524,-0.0940281636$

$\mathrm{H}, 0,-0.0188429382,-1.6112698086,-0.1151887817$

C, $0,-0.1235655949,2.1055599249,-1.0848300462$

$\mathrm{C}, 0,0.8994967332,1.9437194796,1.0200601687$

C, $0,0.1070013218,2.8871003333,0.1704958423$

$\mathrm{H}, 0,-0.8352140507,3.1905063984,0.6518440738$

$\mathrm{H}, 0,0.6544382855,3.8220941041,-0.0236606171$

$\mathrm{H}, 0,-0.677209472,2.4749544794,-1.9398328351$

$\mathrm{H}, 0,1.24744188,2.1703384567,2.0208531176$

$\mathrm{E}\left(\mathrm{B} 3 \mathrm{LYP} / 6-31 \mathrm{G}^{*}\right.$ opt $)=$

$-540.954565$

Sum of electronic and zero-point Energies $=\quad-540.730154$

Sum of electronic and thermal Energies=

$-540.718459$

$-540.717515$

Sum of electronic and thermal Free Energies $=\quad-540.767364$

$\mathrm{E}\left(\mathrm{B} 3 \mathrm{LYP} / 6-311+\mathrm{G}^{* *} / / \mathrm{B} 3 \mathrm{LYP} / 6-31 \mathrm{G}^{*}\right)=$

$-541.083311$

$\chi=-89.7$

68. [11]Annulenium, mono-trans planar-indene cation (43, NImag=2)

$\begin{array}{rrrr}6 & 2.141771 & 0.036850 & -0.000113 \\ 6 & 2.755792 & 1.268658 & -0.000279 \\ 6 & 2.008322 & 2.446521 & -0.000208 \\ 6 & 0.623387 & 2.629397 & 0.000023 \\ 6 & -0.601751 & 1.909537 & 0.000269 \\ 6 & -1.340983 & 0.694710 & 0.000456 \\ 6 & -1.327843 & -0.776819 & 0.000525 \\ 6 & -0.513971 & -1.973915 & 0.000443 \\ 6 & 0.734129 & -2.618457 & 0.000259 \\ 6 & 2.124237 & -2.375124 & 0.000007 \\ 6 & 2.813860 & -1.176741 & -0.000170 \\ 1 & 3.840960 & 1.334401 & -0.000686 \\ 1 & 2.574086 & 3.374921 & -0.000641 \\ 1 & 0.394265 & 3.692464 & -0.000222 \\ 1 & -1.353516 & 2.694674 & 0.000358 \\ 1 & -1.227546 & -2.789758 & 0.000678\end{array}$




\begin{tabular}{|c|c|c|c|}
\hline 1 & 0.562568 & -3.692702 & 0.000231 \\
\hline 1 & 2.724688 & -3.281192 & -0.000115 \\
\hline 1 & 3.900989 & -1.190862 & -0.000429 \\
\hline 1 & 1.061975 & 0.010565 & 0.000197 \\
\hline 6 & -2.747360 & -1.187948 & -0.000551 \\
\hline 6 & -2.835093 & 1.092326 & 0.000604 \\
\hline 6 & -3.606017 & -0.158182 & -0.001124 \\
\hline 1 & -3.038876 & -2.231544 & -0.001163 \\
\hline 1 & -3.061116 & 1.718120 & -0.875987 \\
\hline 1 & -4.687747 & -0.218675 & -0.002648 \\
\hline 1 & -3.061598 & 1.714720 & 0.879581 \\
\hline
\end{tabular}

$\mathrm{E}\left(\mathrm{B} 3 \mathrm{LYP} / 6-31 \mathrm{G}^{*}\right.$ opt $)=$

Sum of electronic and zero-point Energies= Sum of electronic and thermal Energies= Sum of electronic and thermal Enthalpies= Sum of electronic and thermal Free Energies= $\mathrm{E}\left(\mathrm{B} 3 \mathrm{LYP} / 6-311+\mathrm{G}^{* *} / / \mathrm{B} 3 \mathrm{LYP} / 6-31 \mathrm{G}^{*}\right)=$ $\chi=-137.2$
-540.935509
-540.708435
-540.698290
-540.697346
-540.744169
-541.063380

69. [11]Annulenium, mono-trans planar-isoindene cation $(\mathbf{4 4}, \mathrm{NImag}=2)$

$\begin{array}{rrrr}6 & 2.158355 & -0.000095 & -0.000058 \\ 6 & 2.797232 & 1.230296 & -0.000111 \\ 6 & 2.074038 & 2.406190 & -0.000073 \\ 6 & 0.672766 & 2.659336 & 0.000019 \\ 6 & -0.552747 & 2.010890 & 0.000107 \\ 6 & -1.373427 & 0.783420 & 0.000173 \\ 6 & -1.373417 & -0.783132 & 0.000186 \\ 6 & -0.552923 & -2.010692 & 0.000142 \\ 6 & 0.672532 & -2.659286 & 0.000065 \\ 6 & 2.073837 & -2.406327 & -0.000031 \\ 6 & 2.797146 & -1.230532 & -0.000090 \\ 1 & 3.882668 & 1.276338 & -0.000039 \\ 1 & 2.663411 & 3.320889 & 0.000002 \\ 1 & 0.506060 & 3.733575 & 0.000090 \\ 1 & -1.286028 & 2.809855 & 0.000571 \\ 1 & -1.286397 & -2.809487 & 0.000725 \\ 1 & 0.505748 & -3.733515 & 0.000173 \\ 1 & 2.663103 & -3.321095 & 0.000040 \\ 1 & 3.882579 & -1.276657 & -0.000012 \\ 1 & 1.076218 & -0.000055 & -0.000138 \\ 6 & -2.706017 & -1.143886 & -0.000181 \\ 6 & -2.706219 & 1.143993 & -0.000196 \\ 6 & -3.627983 & -0.000088 & -0.000216 \\ 1 & -4.300158 & -0.000178 & 0.874336 \\ 1 & -4.301433 & -0.000194 & -0.873675 \\ 1 & -3.062162 & -2.166311 & -0.000932 \\ 1 & -3.062646 & 2.166313 & 0.000436\end{array}$

$\mathrm{E}\left(\mathrm{B} 3 \mathrm{LYP} / 6-31 \mathrm{G}^{*} \mathrm{opt}\right)=$

$-540.909618$

Sum of electronic and zero-point Energies $=\quad-540.683378$

Sum of electronic and thermal Energies $=\quad-540.673131$

Sum of electronic and thermal Enthalpies $=\quad-540.672187$

Sum of electronic and thermal Free Energies $=\quad-540.719069$

$\mathrm{E}\left(\mathrm{B} 3 \mathrm{LYP} / 6-311+\mathrm{G}^{* *} / / \mathrm{B} 3 \mathrm{LYP} / 6-31 \mathrm{G}^{*}\right)=\quad-541.037806$ 
$\chi=-60.0$

70. [11]Annulenium, all cis-indene cation (45)

C, $0,-0.2053506201,-1.6799347125,-1.9237076592$

C, $0,-0.2755628622,-1.7634551366,2.1819763348$

C, $0,1.004573439,-1.7973137616,1.5257225672$

C, $0,1.0827079253,0.459504373,-1.7173095589$

$\mathrm{C}, 0,-1.485026327,0.0917724456,0.9194125255$

C, $0,-0.6722090898,1.015593241,0.2515317183$

C, $0,0.4913403976,1.1576232805,-0.5809183021$

C, $0,-1.3786542797,-1.0732744348,1.7548738431$

C, $0,0.6850749081,-0.6428492993,-2.4028769428$

C, $0,1.1042889787,-1.9535453663,0.1869712803$

C, $0,-0.0292684041,-2.2431154929,-0.7141436821$

$\mathrm{H}, 0,1.8550991732,1.0543249147,-2.1983061527$

$\mathrm{H}, 0,1.101712848,-0.7712457603,-3.4023242615$

$\mathrm{H}, 0,-0.9669883024,-2.0674179827,-2.5972862977$

$\mathrm{H}, 0,-0.7175027851,-3.0233670279,-0.3944657165$

$\mathrm{H}, 0,2.0973724623,-1.9729765158,-0.258887544$

H, $0,1.904845433,-1.8008349039,2.1368493825$

$\mathrm{H}, 0,-0.3843344879,-2.3391373851,3.1013903555$

$\mathrm{H}, 0,-2.3032369449,-1.2808720625,2.2872682676$

$\mathrm{H}, 0,-2.4683338916,0.5363648844,1.0881622102$

C, $0,-1.1112045329,2.4622223743,0.5991391396$

C, $0,0.9474150044,2.5299141154,-0.4580648071$

C, $0,0.0543138501,3.2883894741,0.2240185796$

$\mathrm{H}, 0,1.8579984453,2.8880082943,-0.9252604588$

$\mathrm{H}, 0,-1.4143245602,2.6010451077,1.6448814342$

$\mathrm{H}, 0,0.1512143166,4.3436788719,0.4542516255$

H,0,-1.9881520304,2.7232429662,-0.0160230614

$\mathrm{E}\left(\mathrm{B} 3 \mathrm{LYP} / 6-31 \mathrm{G}^{*}\right.$ opt $)=$

$-540.933521$

Sum of electronic and zero-point Energies=

Sum of electronic and thermal Energies=

Sum of electronic and thermal Enthalpies=

$-540.709092$

$-540.697283$

$-540.696339$

Sum of electronic and thermal Free Energies=

$-540.746943$

$\mathrm{E}\left(\mathrm{B} 3 \mathrm{LYP} / 6-311+\mathrm{G}^{* *} / / \mathrm{B} 3 \mathrm{LYP} / 6-31 \mathrm{G}^{*}\right)=$

$\chi=-62.0$

$-541.062880$

71. [11]Annulenium, all cis-isoindene cation (46)

C,0,0.115972181,-1.9562850389,-1.9605938793

C, $, 0,-0.4495319536,-1.4159789037,2.2855625032$

C, $0,0.7783566004,-1.6356743234,1.7426507195$

C, $0,0.6905019282,0.5208689682,-2.0174031338$

C, $0,-1.6633441744,-0.0384526245,0.5770857355$

C, $0,-0.6339043815,1.0067693249,0.3249160144$

C, $0,0.2683373216,1.2550568131,-0.852638874$

C, $0,-1.6246093481,-1.0139512105,1.5088917907$

C, $0,0.5233160368,-0.7557212769,-2.5547422179$

C, $0,0.9847745889,-1.6119904533,0.3178950224$

C, $0,0.1444248749,-2.2334336194,-0.5880219872$

$\mathrm{H}, 0,1.2423829471,1.1727099474,-2.6945399594$

$\mathrm{H}, 0,0.7460567563,-0.8167875159,-3.6193431556$

$\mathrm{H}, 0,-0.2655930787,-2.7297399164,-2.6260033952$ 
$\mathrm{H}, 0,-0.5312985195,-3.00122963,-0.2178528536$

$\mathrm{H}, 0,1.8819636357,-1.1244054258,-0.0647686728$

$\mathrm{H}, 0,1.6428874275,-1.7549776301,2.3927253974$

$\mathrm{H}, 0,-0.5688767734,-1.5074202311,3.3648103965$

$\mathrm{H}, 0,-2.5504928425,-1.5465486464,1.7264531778$

$\mathrm{H}, 0,-2.6018704533,0.0981587988,0.038553029$

C, $0,-0.5208536689,2.0702827378,1.1554218331$

C, $0,0.8425680609,2.4951966885,-0.6790578271$

C, $0,0.4284773133,3.0944781548,0.6149928542$

$\mathrm{H}, 0,1.5234115142,2.9685969669,-1.3782526306$

$\mathrm{H}, 0,-1.0807619613,2.1998729759,2.0741652139$

$\mathrm{H}, 0,1.2945180238,3.2722020366,1.272695599$

$\mathrm{H}, 0,-0.0392389534,4.08257685,0.4816065313$

$\mathrm{E}\left(\mathrm{B} 3 \mathrm{LYP} / 6-31 \mathrm{G}^{*}\right.$ opt $)=$

Sum of electronic and zero-point Energies=

Sum of electronic and thermal Energies=

Sum of electronic and thermal Enthalpies=

Sum of electronic and thermal Free Energies=

$\mathrm{E}\left(\mathrm{B} 3 \mathrm{LYP} / 6-311+\mathrm{G}^{* *} / / \mathrm{B} 3 \mathrm{LYP} / 6-31 \mathrm{G}^{*}\right)=$

$\chi=-74.9$
$-540.943581$

$-540.719133$

$-540.707564$

$-540.706620$

$-540.756072$

$-541.072537$

72. [11]Annulenium, all cis-indene cation isomer (47)

C, $0,0.136939707,-1.975915704,-1.9000787763$

C, $, 0,-0.5767857254,-1.2929117976,2.288393677$

C, $0,0.6872345865,-1.5414486903,1.8281063147$

C, $0,0.5382841437,0.5641725322,-2.0497470386$

C, $, 0,-1.5852481189,-0.0392869101,0.3890266148$

C, $0,-0.5625566266,0.969148251,0.2424600994$

C, $0,0.2064751856,1.2924281629,-0.9120950214$

C, $0,-1.6845230583,-0.9309925981,1.4152392791$

C, $0,0.3232301345,-0.7672999814,-2.5343313902$

C, $0,0.9986318738,-1.6256060857,0.4173519738$

C, $0,0.1839165563,-2.2291259264,-0.4950264633$

$\mathrm{H}, 0,1.0911101741,1.1628678151,-2.7770523087$

$\mathrm{H}, 0,0.372897458,-0.8251412536,-3.6214556158$

$\mathrm{H}, 0,-0.1428700928,-2.8113292639,-2.5406466921$

$\mathrm{H}, 0,-0.5508283935,-2.9455205627,-0.1258163437$

$\mathrm{H}, 0,1.9101822085,-1.1375016255,0.0711214027$

H, $0,1.4944120977,-1.6607057113,2.5494737323$

$\mathrm{H}, 0,-0.7820182399,-1.4148415485,3.3516170237$

$\mathrm{H}, 0,-2.6250084512,-1.4656992704,1.5523108282$

$\mathrm{H}, 0,-2.335736901,-0.0743029959,-0.4014207727$

C, $0,-0.2671915913,1.9409718157,1.2700081912$

C, $0,0.8820358369,2.6301277299,-0.6489848961$

C, $0,0.543515413,2.912820128,0.7745054349$

$\mathrm{H}, 0,1.9665994906,2.6054558115,-0.8245594679$

$\mathrm{H}, 0,0.8768045257,3.7948311409,1.3097556304$

$\mathrm{H}, 0,-0.7067073175,1.9131923029,2.2600492692$

$\mathrm{H}, 0,0.4874135403,3.4162096059,-1.3123446794$

$\mathrm{E}\left(\mathrm{B} 3 \mathrm{LYP} / 6-31 \mathrm{G}^{*}\right.$ opt $)=$

$-540.956363$

Sum of electronic and zero-point Energies=

$-540.732057$

Sum of electronic and thermal Energies=

$-540.720529$

Sum of electronic and thermal Enthalpies=

$-540.719585$ 
Sum of electronic and thermal Free Energies= $\mathrm{E}\left(\mathrm{B} 3 \mathrm{LYP} / 6-311+\mathrm{G}^{* *} / / \mathrm{B} 3 \mathrm{LYP} / 6-31 \mathrm{G}^{*}\right)=$ $\chi=-92.7$
$-540.768758$

$-541.084918$

73. [11]Annulenium, all cis tub-indene cation $(\mathbf{4 8}, \mathrm{NImag}=1)$

$\begin{array}{rrrr}6 & -1.189492 & 0.690575 & -0.165196 \\ 6 & 1.866824 & -1.916043 & -0.215464 \\ 6 & 0.892084 & -1.870304 & -1.265114 \\ 6 & 0.761300 & 1.978777 & -1.215000 \\ 6 & 1.838723 & 0.022134 & 1.521207 \\ 6 & 2.006346 & 1.286251 & 0.951034 \\ 6 & 1.818425 & 1.907409 & -0.289381 \\ 6 & 2.015740 & -1.291802 & 1.000610 \\ 6 & -0.565430 & 1.623329 & -1.032145 \\ 6 & -0.425828 & -1.518479 & -1.202460 \\ 6 & -1.176313 & -0.711216 & -0.268837 \\ 1 & 0.935096 & 2.683601 & -2.024232 \\ 1 & -1.266489 & 2.283027 & -1.548984 \\ 1 & -1.062776 & -1.992063 & -1.952189 \\ 1 & 1.152071 & -2.491486 & -2.119275 \\ 1 & 2.517232 & -2.784519 & -0.328751 \\ 1 & 2.552014 & -1.909135 & 1.723425 \\ 1 & 1.992486 & 0.042961 & 2.599322 \\ 1 & 2.501031 & 1.962746 & 1.653141 \\ 1 & 2.555355 & 2.692257 & -0.469274 \\ 6 & -2.450801 & 1.075667 & 0.594971 \\ 6 & -2.344639 & -1.244435 & 0.427368 \\ 6 & -3.053146 & -0.235726 & 0.980146 \\ 1 & -3.127238 & 1.653979 & -0.051364 \\ 1 & -2.560195 & -2.304192 & 0.498113 \\ 1 & -2.237293 & 1.722429 & 1.458506 \\ 1 & -3.914062 & -0.336420 & 1.631134\end{array}$

$\mathrm{E}\left(\mathrm{B} 3 \mathrm{LYP} / 6-31 \mathrm{G}^{*}\right.$ opt $)=$

Sum of electronic and zero-point Energies=

Sum of electronic and thermal Energies=

Sum of electronic and thermal Enthalpies=

Sum of electronic and thermal Free Energies=

$\mathrm{E}\left(\mathrm{B} 3 \mathrm{LYP} / 6-311+\mathrm{G}^{* *} / / \mathrm{B} 3 \mathrm{LYP} / 6-31 \mathrm{G}^{*}\right)=$ $\chi=-109.5$
$-540.928601$

$-540.704431$

$-540.693064$

$-540.692120$

$-540.742333$

$-541.058282$

74. [11]Annulenium, all cis tub-isoindene cation (49, NImag=1)

$\begin{array}{rrrr}6 & 1.219608 & -0.775908 & -0.418538 \\ 6 & -1.680096 & 2.001497 & -0.137026 \\ 6 & -0.853958 & 1.929268 & -1.306992 \\ 6 & -0.925732 & -1.923484 & -1.237581 \\ 6 & -1.531449 & 0.031493 & 1.582244 \\ 6 & -1.810579 & -1.265215 & 1.111748 \\ 6 & -1.792889 & -1.929151 & -0.103238 \\ 6 & -1.689569 & 1.348382 & 1.082177 \\ 6 & 0.411291 & -1.627165 & -1.283987 \\ 6 & 0.437256 & 1.515757 & -1.379285 \\ 6 & 1.188780 & 0.731730 & -0.400035 \\ 1 & -1.283530 & -2.544608 & -2.055760\end{array}$




$\begin{array}{rrrr}1 & 0.941900 & -2.064392 & -2.131803 \\ 1 & 0.964800 & 1.748587 & -2.305656 \\ 1 & -1.226735 & 2.501258 & -2.154091 \\ 1 & -2.319153 & 2.886668 & -0.147549 \\ 1 & -2.070128 & 1.994079 & 1.876664 \\ 1 & -1.535302 & 0.046652 & 2.672241 \\ 1 & -2.240287 & -1.877328 & 1.907136 \\ 1 & -2.489019 & -2.769062 & -0.138911 \\ 6 & 2.155955 & -1.184643 & 0.475841 \\ 6 & 2.098636 & 1.164756 & 0.500453 \\ 6 & 2.775152 & -0.002154 & 1.151976 \\ 1 & 3.864741 & 0.027655 & 0.992193 \\ 1 & 2.640823 & -0.026295 & 2.243647 \\ 1 & 2.395420 & -2.215014 & 0.711680 \\ 1 & 2.342034 & 2.200816 & 0.703668\end{array}$

$\mathrm{E}\left(\mathrm{B} 3 \mathrm{LYP} / 6-31 \mathrm{G}^{*}\right.$ opt $)=$

$-540.914782$

Sum of electronic and zero-point Energies=

$-540.691092$

Sum of electronic and thermal Energies=

$-540.679702$

Sum of electronic and thermal Enthalpies=

$-540.678757$

Sum of electronic and thermal Free Energies=

$-540.728393$

$\mathrm{E}\left(\mathrm{B} 3 \mathrm{LYP} / 6-311+\mathrm{G}^{* *} / / \mathrm{B} 3 \mathrm{LYP} / 6-31 \mathrm{G}^{*}\right)=$

$-541.044446$

$\chi=-84.2$

75. [11]Trannulenium-indene cation (50)

C, $0,-1.2638311145,-0.9866769844,-0.642083777$

C, $0,-1.2105073247,-1.0192576861,0.7861454121$

C, $0,1.2740461678,-1.0199149191,0.7472003858$

C, $0,2.0488378756,0.1153426029,0.6604187482$

C, $0,2.5187105593,0.4089998601,-0.6604055701$

C, $0,-1.668786674,0.1118239423,-1.380003743$

C, $, 0,-0.8101926131,0.3991440709,-2.4838893527$

C, $0,-0.0476178623,1.5700473376,-2.4487424207$

C, $0,1.3336722835,1.2826288289,-2.4643159038$

C,0,2.0531810253,1.5672803194,-1.2797940837

C, $0,0.0239946887,-1.0269080423,1.4713996088$

$\mathrm{H}, 0,-0.5971713554,-1.6802335532,-1.1535058282$

H,0,1.3824943612,-1.754112583,-0.049830991

$\mathrm{H}, 0,1.8919280803,0.9311296952,1.3630045017$

H, $0,2.7855289713,-0.4379392951,-1.2914309937$

$\mathrm{H}, 0,-2.2108560338,0.9229966485,-0.8999063525$

$\mathrm{H}, 0,-0.4035634916,-0.4523616854,-3.0281584078$

$\mathrm{H}, 0,-0.4045146096,2.4166798263,-1.8658677042$

H,0,1.6759444084, $0.4513345969,-3.0792712442$

$\mathrm{H}, 0,1.7391083718,2.4074816893,-0.6637318423$

C, $0,-2.2790093103,-0.5830144516,1.761789315$

C, $0,-0.2079755959,-0.6107375626,2.8521245282$

C,0,-1.5268857227,-0.394270894,3.0461506156

$\mathrm{H}, 0,-3.0790403354,-1.3329317331,1.8494501305$

$\mathrm{H}, 0,0.5764205963,-0.5447245874,3.5972126233$

$\mathrm{H}, 0,-2.7764675263,0.3424668942,1.4371417093$

$\mathrm{H}, 0,-2.0056297328,-0.1567044448,3.9889318228$

$\mathrm{E}\left(\mathrm{B} 3 \mathrm{LYP} / 6-31 \mathrm{G}^{*}\right.$ opt $)=$

$-540.888246$

Sum of electronic and zero-point Energies=

$-540.663177$ 
Sum of electronic and thermal Energies= Sum of electronic and thermal Enthalpies= Sum of electronic and thermal Free Energies= $\mathrm{E}\left(\mathrm{B} 3 \mathrm{LYP} / 6-311+\mathrm{G}^{* *} / / \mathrm{B} 3 \mathrm{LYP} / 6-31 \mathrm{G}^{*}\right)=$ $\chi=-116.5$

76. [11]Trannulenium-isoindene cation (51)

C, $0,-1.3947478641,-0.8184451506,-0.5803610819$ C, $0,-1.3411499372,-0.8334748943,0.8846440333$ C, $0,1.2565954733,-0.8205459042,0.8374888443$ C, $0,1.9687019443,0.3090498516,0.5599219611$ C,0,2.5079367868,0.3949325611,-0.7726176698 C, $0,-1.5586935722,0.3115267125,-1.3263018021$ C, $0,-0.7498216324,0.3972024563,-2.5146659573$ C, $0,0.0719189863,1.5047274848,-2.682025664$ C, $0,1.4279957685,1.098857667,-2.6693896388$ C,0,2.1918719056,1.5032093921,-1.5483052416 C, $0,0.0083320167,-0.8346815752,1.6063360479$ $\mathrm{H}, 0,-0.983442095,-1.69579587,-1.0849985692$ $\mathrm{H}, 0,1.4473593938,-1.6979327675,0.2150964402$ $\mathrm{H}, 0,1.7676894656,1.2278230885,1.1053892762$ $\mathrm{H}, 0,2.7562254558,-0.542641178,-1.2694677067$ $\mathrm{H}, 0,-1.9001288193,1.2303616005,-0.8559323075$ $\mathrm{H}, 0,-0.4753757552,-0.5404202475,-2.9974636879$ $\mathrm{H}, 0,-0.1945610167,2.4375765648,-2.1901782389$ $\mathrm{H}, 0,1.7042630368,0.1808553255,-3.187206285$ $\mathrm{H}, 0,1.931703865,2.4360129435,-1.053002892$ C, $0,-2.3123771138,-0.7555031453,1.8191493981$ C, $0,-0.2296015515,-0.7573614867,2.9330012912$ C,0,-1.7066393341,-0.7248409652,3.1907296886 $\mathrm{H}, 0,-3.37814568,-0.7425212789,1.6243778616$ $\mathrm{H}, 0,0.524132825,-0.7457889214,3.7112787452$

$\mathrm{H}, 0,-2.0379603275,-1.5728713486,3.8087853847$

H,0,-2.0036916052,0.1774240647,3.7476967252

$\mathrm{E}\left(\mathrm{B} 3 \mathrm{LYP} / 6-31 \mathrm{G}^{*}\right.$ opt $)=$ Sum of electronic and zero-point Energies= Sum of electronic and thermal Energies= Sum of electronic and thermal Enthalpies= Sum of electronic and thermal Free Energies= $\mathrm{E}\left(\mathrm{B} 3 \mathrm{LYP} / 6-311+\mathrm{G}^{* *} / / \mathrm{B} 3 \mathrm{LYP} / 6-31 \mathrm{G}^{*}\right)=$ $\chi=-92.1$
$-540.651948$ $-540.651004$ $-540.699485$

$-541.017458$

77. Cyclopropa[1,2][11] annulenium cation, planar (NImag=2: 95i, 100i) (52a)

$\begin{array}{llll}\mathrm{C} & 0.000000 & 0.000000 & 0.000000 \\ \mathrm{C} & 0.000000 & 0.000000 & 1.365478 \\ \mathrm{C} & 0.439405 & 0.000000 & 2.668680 \\ \mathrm{C} & 1.680609 & 0.000000 & 3.317294 \\ \mathrm{C} & 3.057450 & 0.000000 & 3.051109 \\ \mathrm{C} & 4.054692 & 0.000000 & 2.051614 \\ \mathrm{C} & 4.412997 & 0.000000 & 0.682710 \\ \mathrm{C} & 4.054855 & 0.000000 & -0.686244 \\ \mathrm{C} & 3.057640 & 0.000000 & -1.685726 \\ \mathrm{C} & 1.680788 & 0.000000 & -1.951673\end{array}$




$\begin{array}{rrrr}\mathrm{C} & 0.439499 & 0.000000 & -1.303129 \\ \mathrm{H} & -0.391126 & 0.000285 & 3.372368 \\ \mathrm{H} & 1.523953 & 0.000236 & 4.394290 \\ \mathrm{H} & 3.571299 & 0.000194 & 4.009970 \\ \mathrm{H} & 4.999093 & 0.000144 & 2.591327 \\ \mathrm{H} & 5.500272 & 0.000055 & 0.682886 \\ \mathrm{H} & 4.999255 & 0.000038 & -1.225961 \\ \mathrm{H} & 3.571385 & 0.000023 & -2.644638 \\ \mathrm{H} & 1.523971 & 0.000072 & -3.028656 \\ \mathrm{H} & -0.390909 & 0.000186 & -2.006949 \\ \mathrm{C} & -1.330822 & -0.000612 & 0.682770 \\ \mathrm{H} & -1.923164 & -0.918511 & 0.683077 \\ \mathrm{H} & -1.923658 & 0.916887 & 0.682667\end{array}$

$\mathrm{E}\left(\mathrm{B} 3 \mathrm{LYP} / 6-31 \mathrm{G}^{*}\right.$ opt $)=$

Sum of electronic and zero-point Energies= Sum of electronic and thermal Energies= Sum of electronic and thermal Enthalpies= Sum of electronic and thermal Free Energies= $\mathrm{E}\left(\mathrm{B} 3 \mathrm{LYP} / 6-311+\mathrm{G}^{* *} / / \mathrm{B} 3 \mathrm{LYP} / 6-31 \mathrm{G}^{*}\right)=$
$-463.442788$

$-463.251260$

$-463.242225$

$-463.241281$

$-463.285846$

$-463.551442$

78. Cyclopropa[1,2][11]annulenium cation, non-planar (52b)

C, $0,-1.3363304985,-0.5370299515,-0.0822140611$

C, $0,0.887505196,1.8841185792,-0.9744824591$

C,0,-0.0448769609,2.6033848186,-0.1200206235

C, $0,0.3442563073,-2.3246748304,-0.8583179204$

C, $0,1.6047427639,0.1554286807,0.7032471754$

C, $0,1.6310010764,-1.171149737,0.9778770965$

C, $0,1.3942763296,-2.2269437529,0.0251186525$

C, $0,1.5500978165,0.7592212344,-0.6375183004$

C, $0,-0.8799937634,-1.5951202157,-0.8414142187$

C, $0,-1.0481743518,2.0340164581,0.604871557$

C, $0,-1.4010450633,0.651756297,0.548742719$

C, $0,-2.6403684454,-0.1467924623,0.6527618856$

$\mathrm{H}, 0,0.3471729676,-3.1743173962,-1.535016982$

$\mathrm{H}, 0,0.0248577186,3.6908076823,-0.1140417619$

$\mathrm{H}, 0,1.0656164043,2.3158138432,-1.9583917364$

$\mathrm{H}, 0,-1.6677497288,-2.0548517324,-1.4412716912$

$\mathrm{H}, 0,2.0807514903,-3.0736727896,0.0555086414$

$\mathrm{H}, 0,-2.8339612063,-0.7709565169,1.5262902933$

$\mathrm{H}, 0,-3.538066908,0.2362073438,0.160535184$

$\mathrm{H}, 0,2.170712452,0.2922877361,-1.3998986831$

$\mathrm{H}, 0,-1.7037050171,2.6629393872,1.1999658125$

$\mathrm{H}, 0,1.9265933832,-1.4826904059,1.9794401069$

$\mathrm{H}, 0,1.7612360064,0.8411421382,1.5349718$

$\mathrm{E}\left(\mathrm{B} 3 \mathrm{LYP} / 6-31 \mathrm{G}^{*}\right.$ opt $)=$

$-463.479170$

Sum of electronic and zero-point Energies=

$-463.291042$

Sum of electronic and thermal Energies=

Sum of electronic and thermal Enthalpies=

$-463.280408$

$-463.279464$

$-463.326858$

E(B3LYP/6-311+G**//B3LYP/6-31G*) =

$-463.588998$

79. Cyclopropa[1,2][11]annulenium cation triplet, non-planar (52t) 
C, $0,0.3071228957,-0.7214304571,-1.3028335493$

C, $0,0.3899004361,-0.5742067018,2.3132549046$

C, $0,1.7262594551,-0.6669981863,1.9030492233$

C, $0,-2.1562630815,0.0562450744,-1.2401592516$

$\mathrm{C}, 0,-0.343934825,1.348808352,0.9468368408$

C, $0,-1.291967163,1.9981265036,0.1301209733$

C, $0,-2.235516578,1.3420232267,-0.6542728313$

C, $0,-0.600258733,0.1556217478,1.6255938183$

C, $0,-1.055100777,-0.7550169051,-1.5879321105$

C, $0,2.2083405397,-0.5529911408,0.5867571606$

C, $0,1.4666084906,-0.5467953508,-0.6028543447$

C, $0,1.6088012553,-1.0418545226,-2.002603662$

H, $,-3.0789891233,-0.2655960812,-1.7180317544$

$\mathrm{H}, 0,2.454019504,-0.9960187653,2.6424483785$

$\mathrm{H}, 0,0.0962467238,-1.1261145534,3.2049730508$

$\mathrm{H}, 0,-1.2976049686,-1.4925577028,-2.3533012668$

$\mathrm{H}, 0,-3.1004332354,1.9248731703,-0.9688308975$

$\mathrm{H}, 0,1.8906703711,-0.3630200921,-2.8114527072$

$\mathrm{H}, 0,1.950574315,-2.0702689512,-2.1488548295$

$\mathrm{H}, 0,-1.6365994565,-0.1597758595,1.7311688697$

$\mathrm{H}, 0,3.2823461141,-0.6584168011,0.4529119519$

$\mathrm{H}, 0,-1.2907304926,3.0875232238,0.1144905015$

$\mathrm{H}, 0,0.5865487593,1.8701825713,1.1647356735$

$\mathrm{E}\left(\mathrm{UB} 3 \mathrm{LYP} / 6-31 \mathrm{G}^{*}\right.$ opt $)=$

Sum of electronic and zero-point Energies=

Sum of electronic and thermal Energies=

Sum of electronic and thermal Enthalpies=

Sum of electronic and thermal Free Energies=

$\mathrm{E}\left(\mathrm{UB} 3 \mathrm{LYP} / 6-311+\mathrm{G}^{* *} / / \mathrm{UB} 3 \mathrm{LYP} / 6-31 \mathrm{G}^{*}\right)=$
$-463.462565$

$-463.275114$

$-463.264736$

$-463.263792$

$-463.311391$

$-463.571212$

80. Dicyclopropa[1,2:6,7][11] annulenium cation, planar (NImag=2: 48.0i, 48.4i) (53a)

C, $0,0.9826848582,-0.0001427466,-1.8199872609$

C, $0,0.9502948188,-0.003660095,2.6116238718$

C, $0,2.1014916725,-0.0036601106,1.8186182238$

$\mathrm{C}, 0,-1.5513234782,-0.0032658812,-2.2520378106$

C, $0,-1.4006058547,0.0024123242,1.4784302923$

$\mathrm{C}, 0,-2.0506966178,-0.0001427517,0.2695673108$

C, $0,-2.3223779173,-0.0021110374,-1.0848019774$

C, $0,-0.4490139197,0.0007000753,2.4798036286$

C, $, 0,-0.1859403925,-0.002111032,-2.5564938289$

C, $0,2.4770046444,0.0007000473,0.4642063478$

C, $0,1.8805017137,0.0024123094,-0.7817711902$

C, $0,-2.8985978589,0.0041368547,1.4984950166$

C, $0,2.4330955813,0.0041368492,-2.1742591272$

$\mathrm{H}, 0,-2.1686243033,-0.0046164886,-3.1481660639$

$\mathrm{H}, 0,2.9906520337,-0.006571085,2.4458540269$

$\mathrm{H}, 0,1.2194739596,-0.0065710581,3.665935728$

$\mathrm{H}, 0,0.0109053144,-0.0028230839,-3.6268121625$

$\mathrm{H}, 0,-3.392573311,-0.0028230959,-1.2823149795$

$\mathrm{H}, 0,-3.4215664263,0.9233623641,1.7726247004$

$\mathrm{H}, 0,-3.4232208729,-0.9123684225,1.7785113184$

$\mathrm{H}, 0,2.8755995673,0.9233623586,-2.5651982892$

$\mathrm{H}, 0,2.8816892682,-0.9123684278,-2.5646462945$

$\mathrm{H}, 0,-0.932322449,0.0024940754,3.4555052648$ 
$\mathrm{E}\left(\mathrm{B} 3 \mathrm{LYP} / 6-31 \mathrm{G}^{*}\right.$ opt $)=$

Sum of electronic and zero-point Energies=

Sum of electronic and thermal Energies=

Sum of electronic and thermal Enthalpies=

Sum of electronic and thermal Free Energies=

$\mathrm{E}\left(\mathrm{B} 3 \mathrm{LYP} / 6-311+\mathrm{G}^{* *} / / \mathrm{B} 3 \mathrm{LYP} / 6-31 \mathrm{G}^{*}\right)=$
$-501.541228$

$-501.346113$

$-501.336561$

$-501.335616$

$-501.380952$

$-501.655699$

81. Dicyclopropa[1,2:6,7][11]annulenium cation, non-planar (53b)

$\mathrm{C}, 0,0.8705504245,0.1887478991,-1.7271483072$

C, $0,0.8578545179,-0.602368431,2.4808891779$

$\mathrm{C}, 0,2.0124371639,-0.6019701798,1.6856788332$

C, $0,-1.4304504815,-0.8260353047,-2.0764805451$

C, $0,-1.2457378276,0.3981411032,1.3679568738$

C, $0,-1.9240390085,0.1890307854,0.1978068933$

$\mathrm{C}, 0,-2.2246786841,-0.4550550986,-0.9862808238$

C, $0,-0.4187902695,-0.0385567736,2.3914976799$

C, $0,-0.1286637579,-0.4553430299,-2.4299653046$

C,0,2.3837973585,-0.0378282908,0.4611201551

C,0,1.7220880998,0.3984158608,-0.6764343628

C,0,-2.6550361746,0.8981997946,1.2983868131

C,0,2.1592769465,0.8981490214,-2.0180883875

$\mathrm{H}, 0,-1.9778480685,-1.3272721151,-2.8711268424$

$\mathrm{H}, 0,2.8690236706,-1.0153315636,2.2146888954$

$\mathrm{H}, 0,1.0468585225,-1.0160623447,3.4696198835$

$\mathrm{H}, 0,0.1175855636,-0.6147054776,-3.4796188574$

H,0,-3.2931929783,-0.6142165967,-1.1303361959

$\mathrm{H}, 0,-2.8094875738,1.9704757007,1.1616589277$

$\mathrm{H}, 0,-3.4430643219,0.3975129409,1.8657248942$

$\mathrm{H}, 0,2.0862340551,1.9703139844,-2.2116133167$

H,0,2.9703980489,0.3974847905,-2.5519087201

$\mathrm{H}, 0,-0.902706074,0.0646705065,3.3636642502$

H,0,3.4645493122,0.0659660386,0.3556149097

$\mathrm{E}\left(\mathrm{B} 3 \mathrm{LYP} / 6-31 \mathrm{G}^{*}\right.$ opt $)=$

$-501.546306$

Sum of electronic and zero-point Energies=

Sum of electronic and thermal Energies=

Sum of electronic and thermal Enthalpies=

Sum of electronic and thermal Free Energies=

$-501.351911$

$-501.340626$

$-501.339682$

$-501.389620$

$\mathrm{E}\left(\mathrm{B} 3 \mathrm{LYP} / 6-311+\mathrm{G}^{* *} / / \mathrm{B} 3 \mathrm{LYP} / 6-31 \mathrm{G}^{*}\right)=$

$-501.661715$

82. Tricyclopropa[1,2:4,5:8,9][11]annulenium cation, planar (54)

C, $0,-2.3053616108,-0.0010835837,-0.0202598089$

$\mathrm{C}, 0,-2.2957887153,-0.0019920627,1.3567147561$

$\mathrm{C}, 0,-1.2678040162,-0.0012415207,2.3171628659$

C, $0,0.1104413197,-0.0002787613,2.2620758009$

C, $0,1.3989860384,0.0004390795,1.7808749665$

C, $0,2.476125155,0.0010013165,0.9192633166$

C, $0,2.6231625833,0.0008892,-0.4798773909$

C, $0,1.7279769881,0.0004652639,-1.5262036993$

C, $0,0.5200705677,0.0000083375,-2.1664009009$

C, $0,-0.8552637259,0.0000083934,-2.2906945805$

C,0,-1.8125616399,0.0010657852,-1.2954231706 
C, $0,1.2194500049,0.0004012904,3.266019475$

C, $0,-3.2983697421,0.0003202739,-1.1431713089$

C, $0,1.7420987152,-0.0000235244,-3.0251428216$

H,0,-3.2932092276,-0.0037958391,1.7929067425

$\mathrm{H}, 0,-1.6411241836,-0.0015189812,3.339663106$

$\mathrm{H}, 0,3.4281948668,0.0015933738,1.4469329157$

$\mathrm{H}, 0,3.6623973442,0.0017959464,-0.8040753768$

$\mathrm{H}, 0,-1.235951675,0.0000939387,-3.3102409542$

$\mathrm{H}, 0,1.426736383,0.9177589352,3.821911982$

$\mathrm{H}, 0,1.4274086866,-0.9166856838,3.8221063912$

$\mathrm{H}, 0,-3.8504152669,0.9171477856,-1.3633463143$

$\mathrm{H}, 0,-3.8499074537,-0.9159853912,-1.3667578538$

$\mathrm{H}, 0,2.013728974,-0.9167083867,-3.5540192544$

H, $0,2.01317002,0.9164273764,-3.5547063803$

$\mathrm{E}\left(\mathrm{B} 3 \mathrm{LYP} / 6-31 \mathrm{G}^{*}\right.$ opt $)=$

$-539.615308$

Sum of electronic and zero-point Energies= $-539.416231$

Sum of electronic and thermal Energies=

$-539.404196$

Sum of electronic and thermal Enthalpies=

$-539.403252$

Sum of electronic and thermal Free Energies=

$-539.455564$

$\mathrm{E}\left(\mathrm{B} 3 \mathrm{LYP} / 6-311+\mathrm{G}^{* *} / / \mathrm{B} 3 \mathrm{LYP} / 6-31 \mathrm{G}^{*}\right)=$

$-539.736333$

83. Tetracyclopropa[1,2:3,4:6,7:8,9][11] annulenium cation, planar (55)

C, $0,0.9698858918,-0.0468936242,-1.9181183909$

C, $0,1.1524589251,-0.110396514,2.6060954347$

C, $0,2.257889458,-0.1929756618,1.7312912533$

C, $0,-1.6792805868,0.123712295,-2.133512266$

C, $0,-1.3578490029,0.1049166185,1.6436648004$

C, $0,-2.0861388347,0.1502524879,0.4985526298$

$\mathrm{C}, 0,-2.2188599304,0.1540580332,-0.8625847912$

$\mathrm{C}, 0,-0.1979585899,0.0073910851,2.3747759879$

C, $0,-0.3201506605,0.0396940686,-2.3638343134$

C, $0,2.3490841774,-0.1805403998,0.3592348009$

C, $0,1.9109787783,-0.1253429704,-0.942459705$

C, $0,-3.4771420608,0.2381954395,-0.0622649511$

C, $0,-1.4867915922,0.1130501523,3.1337211925$

C, $0,3.3856046939,-0.2439325137,-0.7223524283$

C, $0,0.7498392457,-0.0224327557,-3.4041003402$

$\mathrm{H}, 0,-2.3513681271,0.1683444656,-2.9868841942$

H,0,-4.1272293954,-0.6379721348,-0.0078758139

$\mathrm{H}, 0,-4.0112482673,1.1900562084,-0.0181519326$

$\mathrm{H}, 0,-1.8852676917,-0.7734200879,3.6320203414$

$\mathrm{H}, 0,-1.7311453227,1.0528672247,3.63368634$

$\mathrm{H}, 0,3.8730637744,-1.1975134232,-0.9367589509$

$\mathrm{H}, 0,4.0159781626,0.6293967083,-0.9036417011$

$\mathrm{H}, 0,0.9942028016,0.8838247315,-3.9628956022$

$\mathrm{H}, 0,0.8770079174,-0.9441173487,-3.9764148094$

$\mathrm{H}, 0,3.2175106256,-0.2799461534,2.2374539581$

H,0,1.4190760508,-0.1440546325,3.6608088834

$\mathrm{E}\left(\mathrm{B} 3 \mathrm{LYP} / 6-31 \mathrm{G}^{*}\right.$ opt $)=$

$-577.673933$

Sum of electronic and zero-point Energies=

$-577.470507$

$-577.457768$

Sum of electronic and thermal Energies=

$-577.456824$

Sum of electronic and thermal Enthalpies=

$-577.509902$ 
$\mathrm{E}\left(\mathrm{B} 3 \mathrm{LYP} / 6-311+\mathrm{G}^{* *} / / \mathrm{B} 3 \mathrm{LYP} / 6-31 \mathrm{G}^{*}\right)=\quad-577.802084$

84. Pentacyclopropa[1,2:3,4:5,6:7,8:9,10][11]annulenium cation, planar (56)

C,0,-2.0622345369,-1.1478025117,-0.0688599354

C, $0,0.6403657174,2.2912231069,0.2164091218$

C, $, 0,-0.7340963738,2.2602096008,0.2442359295$

C, $0,0.0574348889,-2.7904589881,-0.2822264246$

C, $0,2.2882360372,0.2849746788,-0.0241197103$

C, $0,2.1076117555,-1.0535129134,-0.1534000302$

C, $0,1.2805163079,-2.1438577449,-0.2438560378$

C,0,1.767272575,1.5499489283,0.1155637328

C,0,-1.1910498584,-2.1997297743,-0.1938815642

C, $0,-1.8294986121,1.4686406637,0.1888055725$

C, $0,-2.2978676389,0.1811745424,0.0696375775$

C, $0,2.7558532774,-2.3960094023,-0.3018276569$

C, $0,3.2588084533,1.4267683374,0.0687324983$

C, $0,-0.0740332814,3.6012976443,0.3650563379$

C, $0,-3.3146076032,1.2779606531,0.2016851744$

C, $0,-2.6546226388,-2.5183080463,-0.1927063679$

H,0,0.0796844981,-3.8726285955,-0.3916062979

$\mathrm{H}, 0,3.206058931,-2.6672833113,-1.2593991113$

H,0,3.2465783059,-2.8489142633,0.5626194294

H,0,3.7891821283,1.7413075656,-0.8323630923

$\mathrm{H}, 0,3.8315344033,1.5575947687,0.98909218$

$\mathrm{H}, 0,-0.1065975004,4.2854544145,-0.485140543$

H,0,-0.0657864702,4.1007030527,1.3358942909

$\mathrm{H}, 0,-3.8941343944,1.5671039813,-0.6773945201$

$\mathrm{H}, 0,-3.8555528618,1.384178093,1.1441779813$

H,0,-3.0891105763,-2.9923849618,0.6902897353

H,0,-3.1303872776,-2.8102433919,-1.1316593576

$\mathrm{E}\left(\mathrm{B} 3 \mathrm{LYP} / 6-31 \mathrm{G}^{*}\right.$ opt $)=$

$-615.721537$

Sum of electronic and zero-point Energies=

Sum of electronic and thermal Energies=

$-615.513598$

$-615.500106$

Sum of electronic and thermal Enthalpies=

$-615.499162$

Sum of electronic and thermal Free Energies=

$-615.553532$

$\mathrm{E}\left(\mathrm{B} 3 \mathrm{LYP} / 6-311+\mathrm{G}^{* *} / / \mathrm{B} 3 \mathrm{LYP} / 6-31 \mathrm{G}^{*}\right)=$

$-615.856752$

85. Cyclopropa[1,2][10]annulene, planar (NImag=1: 15i) (57)

C, $0,-1.6782573946,0.0007468529,-1.7236958309$

C, $0,-1.3705167846,0.0019969578,1.9991358463$

C, $0,0.9478500273,0.0026226191,-2.2307860838$

C, $0,1.0866173576,-0.0027203277,1.3675182955$

C, $0,1.7373520341,-0.0021195123,0.1802604662$

C, $0,1.8769578629,0.0001081488,-1.1854026511$

C, $0,0.0105413958,-0.0004051909,2.2199178479$

$\mathrm{C}, 0,-0.4411601733,0.0025774887,-2.4035141823$

C, $0,-2.2633823985,0.0018459518,0.9211874543$

C, $0,-2.3558527324,0.0004051887,-0.4873613952$

C, $0,2.5882495335,-0.0051783486,1.4185936483$

H,0,1.4522876831,0.0044338359,-3.1968038697

H,0,-1.9134424019,0.0037078806,2.9440588002

H,0,-0.6509915098,0.0039566701,-3.4730741688

H,0,2.9138525103,0.0000006282,-1.519442872 
H,0,3.1126387713,-0.9215147482, 1.7055260084

H,0,3.1156381609,0.9086187519,1.7081291168

$\mathrm{H}, 0,0.286805792,-0.0008086428,3.2736784658$

$\mathrm{H}, 0,-3.2778621691,0.002870481,1.3197102505$

$\mathrm{H}, 0,-3.4198838655,-0.0005843187,-0.723120883$

$\mathrm{H}, 0,-2.4494353377,0.0000404914,-2.4937813387$

$\mathrm{E}\left(\mathrm{B} 3 \mathrm{LYP} / 6-31 \mathrm{G}^{*}\right.$ opt $)=$

Sum of electronic and zero-point Energies=

Sum of electronic and thermal Energies=

Sum of electronic and thermal Enthalpies=

Sum of electronic and thermal Free Energies=

$\mathrm{E}\left(\mathrm{B} 3 \mathrm{LYP} / 6-311+\mathrm{G}^{* *} / / \mathrm{B} 3 \mathrm{LYP} / 6-31 \mathrm{G}^{*}\right)=$

86. Cyclopropa[1,2][10]annulene, non-planar (57a)

C,0,-1.66425616,-0.1493614914,-1.7149948795

C, $0,-1.3662530146,0.1762092059,1.985368659$

C,0,0.9385243514,0.1769694921,-2.2197368003

C, $0,1.0799906323,-0.0679088219,1.3645247079$

C, $0,1.7312636877,-0.0677271296,0.1762716204$

C, $0,1.8712589898,0.080674119,-1.1806522304$

C, $0,0.0114615563,0.0800839404,2.2125820442$

C,0,-0.4433780398,0.055715969,-2.3951545246

C, $0,-2.2575427777,0.0551024059,0.9148255433$

C, $0,-2.3409865993,-0.1495932686,-0.4802929503$

C, $0,2.5768857873,-0.1865692566,1.4123307768$

$\mathrm{H}, 0,1.4362913436,0.3256658284,-3.1777480176$

$\mathrm{H}, 0,-1.9060470409,0.324550617,2.9204007709$

$\mathrm{H}, 0,-0.6596895175,0.0981513008,-3.4628534019$

$\mathrm{H}, 0,2.9076896213,0.1230770917,-1.5138393388$

$\mathrm{H}, 0,3.0252446112,-1.1537163923,1.6579259466$

H,0,3.1762771418,0.6679010983,1.7409783855

$\mathrm{H}, 0,0.2881817489,0.122259192,3.265504991$

$\mathrm{H}, 0,-3.2739438383,0.0972514024,1.3069031871$

$\mathrm{H}, 0,-3.3949774767,-0.3135260591,-0.7051839218$

$\mathrm{H}, 0,-2.4208370736,-0.3131850646,-2.4825203999$

$\mathrm{E}\left(\mathrm{B} 3 \mathrm{LYP} / 6-31 \mathrm{G}^{*}\right.$ opt $)=$

Sum of electronic and zero-point Energies=

Sum of electronic and thermal Energies=

Sum of electronic and thermal Enthalpies=

Sum of electronic and thermal Free Energies=

$\mathrm{E}\left(\mathrm{B} 3 \mathrm{LYP} / 6-311+\mathrm{G}^{* *} / / \mathrm{B} 3 \mathrm{LYP} / 6-31 \mathrm{G}^{*}\right)=$

87. Tricyclopropa[1,2:4,5:7,8][10]annulene, planar (58)

$\mathrm{C}, 0,-1.2792315054,0.1024075744,-1.6067760665$

C,0,-1.0661459418,0.050987129,1.7891725111

C, $0,1.3599641578,-0.0670547046,-2.0731846228$

C, $0,1.3693646857,-0.1077037582,1.5308187428$

C, $0,2.0676166472,-0.1406539773,0.3647046455$

C, $0,2.2646563274,-0.1386176221,-1.001096635$

C,0,0.2187996121,-0.0399782156,2.2916043433

C, $0,-0.0165832539,0.0243627748,-2.1046608095$

C,0,-1.9365552668,0.1200428501,0.7578179512
$-425.026491$

$-424.852725$

$-424.844261$

$-424.843317$

$-424.886761$

$-425.135680$
$-425.026510$

$-424.852855$

$-424.843476$

$-424.842532$

$-424.889073$

$-425.135830$ 
C, $0,-2.2183316973,0.1533393823,-0.5954340522$

C, $0,2.8610641133,-0.2075437712,1.6427509771$

C, $0,-2.5243638549,0.143404596,2.1408667702$

C, $0,-1.1433477413,0.1096861364,-3.0999191835$

$\mathrm{H}, 0,1.827891514,-0.0872699368,-3.0566010227$

$\mathrm{H}, 0,-1.4267397287,-0.7815964589,-3.6682101527$

$\mathrm{H}, 0,-1.3061955677,1.0426373983,-3.6483097671$

$\mathrm{H}, 0,-2.9239275906,1.0807889569,2.5406345504$

H,0,-3.0442659178,-0.7419494165,2.5203499736

$\mathrm{H}, 0,-3.263159924,0.2255608175,-0.8922343757$

$\mathrm{H}, 0,0.3377575156,-0.0599368347,3.3734880344$

$\mathrm{H}, 0,3.3133102463,-1.157073221,1.9453577565$

$\mathrm{H}, 0,3.4338749293,0.667167719,1.965971599$

H, $0,3.3100168308,-0.2043993887,-1.3004340254$

$\mathrm{E}\left(\mathrm{B} 3 \mathrm{LYP} / 6-31 \mathrm{G}^{*}\right.$ opt $)=$

$-501.172082$

Sum of electronic and zero-point Energies=

$-500.990081$

Sum of electronic and thermal Energies=

$-500.979452$

Sum of electronic and thermal Enthalpies=

$-500.978508$

Sum of electronic and thermal Free Energies=

$-501.026128$

$\mathrm{E}\left(\mathrm{B} 3 \mathrm{LYP} / 6-311+\mathrm{G}^{* *} / / \mathrm{B} 3 \mathrm{LYP} / 6-31 \mathrm{G}^{*}\right)=$

$-501.292409$

88. Tetracyclopropa[1,2:3,4:6,7:8,9][10]annulene, planar (59)

$\mathrm{C}, 0,-1.2760594397,0.1018054322,-1.5722095024$

C, $0,-1.3002106912,0.0632143917,2.14986292$

C, $0,1.3002872087,-0.0625564982,-2.1498487354$

C, $0,1.2761654047,-0.102496931,1.5723087055$

C, $0,1.9820231768,-0.135851471,0.4052037009$

C, $0,1.9896369753,-0.1215751431,-0.9519641751$

C, $0,0.0788427141,-0.0292597998,2.2073267254$

C, $0,-0.0787762884,0.0295529765,-2.207408827$

C, $0,-1.9895873963,0.1221625537,0.9519346751$

C, $0,-1.9822226053,0.1351901978,-0.4052966241$

C, $0,3.3248036944,-0.217307035,-0.2695107243$

C, $0,1.3008096409,-0.1196604291,3.076973245$

$\mathrm{C}, 0,-3.3249018027,0.2171188138,0.269520874$

C, $0,-1.3008244237,0.1195638054,-3.0769050289$

$\mathrm{H}, 0,1.8632141279,-0.0899650175,-3.080576106$

$\mathrm{H}, 0,3.9863841314,0.6540997872,-0.2556294634$

$\mathrm{H}, 0,3.8660784946,-1.1682038815,-0.2757057836$

$\mathrm{H}, 0,1.6433145567,0.7672887545,3.6184862872$

$\mathrm{H}, 0,1.5220245981,-1.0550041429,3.599852718$

$\mathrm{H}, 0,-3.8662465447,1.1679805103,0.2746789696$

$\mathrm{H}, 0,-3.9864132977,-0.6543532773,0.2565173541$

$\mathrm{H}, 0,-1.6427916506,-0.7671440861,-3.6191382334$

$\mathrm{H}, 0,-1.5225403077,1.055181773,-3.5991058537$

H,0,-1.8629411138,0.0907143969,3.0806967392

$\mathrm{E}\left(\mathrm{B} 3 \mathrm{LYP} / 6-31 \mathrm{G}^{*}\right.$ opt $)=$

$-539.218696$

Sum of electronic and zero-point Energies=

Sum of electronic and thermal Energies=

$-539.032050$

$-539.020610$

$-539.019666$

Sum of electronic and thermal Enthalpies=

Sum of electronic and thermal Free Energies= $-539.069005$

$\mathrm{E}\left(\mathrm{B} 3 \mathrm{LYP} / 6-311+\mathrm{G}^{* *} / / \mathrm{B} 3 \mathrm{LYP} / 6-31 \mathrm{G}^{*}\right)=$

$-539.345273$ 
89. Tricyclopropa[1,2:5,6:9,10][12]annulene, planar (60)

C, $0,-0.5780946321,-0.0267923689,-2.4155856648$

C, $0,0.7298893068,-0.0137228689,-2.7232357862$

C, $0,1.9903598758,0.0024766419,-1.996839981$

C, $0,2.3800463246,0.0184723285,-0.7109458756$

C, $0,2.3809846568,0.0324607396,0.70698477$

C, $0,1.9933274884,0.0423394112,1.9935545733$

C, $0,0.7339260534,0.0376626847,2.7220528723$

C, $0,-0.574532636,0.0193582427,2.416756929$

C, $0,-1.80294915,-0.0039158587,1.7085709996$

C, $0,-2.7232428813,-0.0285014615,0.7297250956$

C, $0,-1.8056072504,-0.0384180137,-1.7056786522$

C, $0,3.6961076836,0.0366287063,-0.0030605898$

C, $0,-1.8503274369,-0.048368274,-3.1995074983$

C, $0,-1.8457818191,0.0130430174,3.2024153049$

$\mathrm{H}, 0,0.9002033991,-0.0171154258,-3.8000967618$

$\mathrm{H}, 0,2.8367821093,0.002851093,-2.6840581091$

$\mathrm{H}, 0,2.8407397604,0.0580206015,2.6793261705$

$\mathrm{H}, 0,0.9057540437,0.052623238,3.7985795084$

$\mathrm{H}, 0,-3.7408794824,-0.0386414389,1.1208820468$

$\mathrm{H}, 0,4.3044719811,-0.8724265975,0.0054837627$

$\mathrm{H}, 0,4.2888328606,0.9559495311,-0.0128412617$

$\mathrm{H}, 0,-2.1377727031,-0.9697001987,-3.7143576859$

$\mathrm{H}, 0,-2.1632667458,0.8585582536,-3.7249570397$

$\mathrm{H}, 0,-2.1565996945,0.9296441491,3.712141028$

$\mathrm{H}, 0,-2.1340534222,-0.8985430346,3.7339115086$

C, $0,-2.7243765055,-0.0427604461,-0.7251281888$

$\mathrm{H}, 0,-3.7425865755,-0.0609950548,-1.1144830149$

$\mathrm{E}\left(\mathrm{B} 3 \mathrm{LYP} / 6-31 \mathrm{G}^{*}\right.$ opt $)=$

Sum of electronic and zero-point Energies=

Sum of electronic and thermal Energies=

Sum of electronic and thermal Enthalpies=

Sum of electronic and thermal Free Energies=

$\mathrm{E}\left(\mathrm{B} 3 \mathrm{LYP} / 6-311+\mathrm{G}^{* *} / / \mathrm{B} 3 \mathrm{LYP} / 6-31 \mathrm{G}^{*}\right)=$
$-578.506473$

$-578.292380$

$-578.279135$

$-578.278191$

$-578.334369$

$-578.649039$

90. Tricyclopropa[1,2:5,6:9,10][12]annulene triplet, planar (NImag=2: 5i, 14i) $(\mathbf{6 0 t - a})$

C, $0,-2.4639367424,0.0121294547,-0.0308900512$

$\mathrm{C}, 0,-2.4692400771,0.01239655,1.3560473601$

C, $0,-1.5094180557,0.0107942491,2.378696838$

$\mathrm{C}, 0,-0.1249088134,0.0044817588,2.4611061403$

C, $0,1.2052090644,-0.0034102332,2.1493916259$

C, $0,2.4089014666,-0.0129120843,1.4604196492$

C, $0,2.8145839815,-0.0191447194,0.1178574405$

C, $0,2.1937681603,-0.0165897389,-1.1223713669$

C, $0,1.2587032705,-0.0097085101,-2.1184877952$

C, $0,0.0602811836,0.0009897952,-2.816500273$

C, $0,-2.0688721172,0.0124517681,-1.3386818622$

C, $0,0.8447033043,0.0029029762,3.6045472468$

C, $0,-3.5438618027,0.0178895141,-1.0707647352$

C,0,2.6991983952,-0.0221266172,-2.5337501065

$\mathrm{H}, 0,-3.4856250823,0.0156292261,1.7478804429$

$\mathrm{H}, 0,-1.9648025365,0.014831803,3.3682485933$

H, $0,3.2566001931,-0.0167532848,2.1445539$ 
$\mathrm{H}, 0,3.899272596,-0.0276381477,0.01764272$

$\mathrm{H}, 0,0.229196531,0.0007508289,-3.8926426875$

$\mathrm{H}, 0,0.986669047,0.9194634929,4.1867444281$

$\mathrm{H}, 0,0.9770522989,-0.9115065958,4.1923759241$

$\mathrm{H}, 0,-4.11562042,0.9358659364,-1.2429532467$

H, $0,-4.1225105243,-0.8950858754,-1.246468515$

$\mathrm{H}, 0,3.1294767071,-0.9416546566,-2.9443181464$

$\mathrm{H}, 0,3.1451641371,0.8892141365,-2.9458443619$

C, $0,-1.305217597,0.0100819709,-2.4965144128$

$\mathrm{H}, 0,-1.9342346732,0.0155263319,-3.3858532374$

$\mathrm{E}\left(\mathrm{UB} 3 \mathrm{LYP} / 6-31 \mathrm{G}^{*}\right.$ opt $)=$

Sum of electronic and zero-point Energies=

Sum of electronic and thermal Energies=

Sum of electronic and thermal Enthalpies=

Sum of electronic and thermal Free Energies=

$\mathrm{E}\left(\mathrm{UB} 3 \mathrm{LYP} / 6-311+\mathrm{G}^{* *} / / \mathrm{UB} 3 \mathrm{LYP} / 6-31 \mathrm{G}^{*}\right)=$
$-578.492332$

$-578.279495$

$-578.268105$

$-578.267160$

$-578.317344$

$-578.633914$

91. Tricyclopropa[1,2:5,6:9,10][12]annulene triplet, non-planar (60t-b)

C, $0,-2.4495036475,0.1283983201,-0.0288444283$

C, $0,-2.4636309113,0.0230223859,1.3535632794$

C, $0,-1.5086662082,-0.1320153466,2.369351041$

C, $0,-0.1239935199,-0.1375381026,2.4483511324$

C, $0,1.1974470686,0.0137810059,2.1389931986$

C, $0,2.3867591551,0.2193786025,1.4551024433$

C, $0,2.789333174,0.2543057075,0.1126414701$

C, $0,2.1796403964,0.0700697926,-1.1200100987$

C, $0,1.2572102306,-0.1412709307,-2.1039372893$

C, $0,0.0597944774,-0.2413940964,-2.7972671008$

C, $0,-2.0553129252,0.0677325063,-1.336105638$

C, $0,0.8508672447,-0.1825641627,3.5858523781$

C, $0,-3.5175680749,0.2671441056,-1.0716607893$

C, $0,2.696100333,-0.0868609488,-2.5203157681$

$\mathrm{H}, 0,-3.4793844333,0.0642755903,1.745507811$

H, $0,-1.9651177692,-0.2413463784,3.3523578469$

$\mathrm{H}, 0,3.2250661934,0.3374939796,2.1407204148$

$\mathrm{H}, 0,3.8625636368,0.4083192186,0.0073318365$

$\mathrm{H}, 0,0.2270584712,-0.3997439329,-3.8618801941$

$\mathrm{H}, 0,0.900765169,0.6650961327,4.2775564011$

$\mathrm{H}, 0,1.0864766494,-1.1430296852,4.0557173194$

$\mathrm{H}, 0,-3.9577198147,1.2525551423,-1.2559839401$

$\mathrm{H}, 0,-4.215529909,-0.5602064891,-1.2387979448$

$\mathrm{H}, 0,3.2201558703,-1.0111084412,-2.7851301517$

H,0,3.0526956539,0.7898215291,-3.0712140156

C, $0,-1.302302169,-0.1215380202,-2.4848453939$

H, $,-1.93407746,-0.166031577,-3.3713960017$

$\mathrm{E}\left(\mathrm{UB} 3 \mathrm{LYP} / 6-31 \mathrm{G}^{*}\right.$ opt $)=$

$-578.492355$

Sum of electronic and zero-point Energies=

Sum of electronic and thermal Energies=

Sum of electronic and thermal Enthalpies=

Sum of electronic and thermal Free Energies=

$\mathrm{E}\left(\mathrm{UB} 3 \mathrm{LYP} / 6-311+\mathrm{G}^{* *} / / \mathrm{UB} 3 \mathrm{LYP} / 6-31 \mathrm{G}^{*}\right)=$

$-578.279592$

$-578.266342$

$-578.265398$

$-578.323055$

$-578.634095$

92. Heptacyclopropa[15]annulenium cation, planar (NImag=2: 10i, 14i) (61) 
C, $0,-3.1262380031,-0.0053233186,-0.9094103194$

C, $0,-3.1094366987,-0.0047596618,0.4473669758$

C, $0,-2.6216252896,-0.0051405571,1.7377063895$

C, $0,-1.6786308709,-0.0039170345,2.705460461$

C, $0,-0.4023809467,-0.0024939292,3.2326766113$

C, $0,2.8752728295,0.0069241289,-1.2744685974$

C, $0,2.0502374052,0.0057424896,-2.3800133999$

C, $0,0.9157385717,0.0034826182,-3.124344277$

C, $0,-0.4257350571,0.000370342,-3.4211810606$

C, $0,-1.8029863272,-0.0027022016,-3.2901770637$

C, $0,-2.6545190709,-0.0046997566,-2.1998147211$

$\mathrm{H}, 0,-4.6957847061,0.9081664022,-2.2270296228$

C,0,3.5308871361,0.0090853421,-2.6199489767

$\mathrm{H}, 0,4.0042337345,-0.9062015457,-2.9820894282$

$\mathrm{H}, 0,-1.7772379418,0.9112411389,4.7477091434$

$\mathrm{H}, 0,-1.774960418,-0.9210297228,4.7470215305$

$\mathrm{H}, 0,0.651402842,-0.913549148,-5.154811596$

$\mathrm{H}, 0,0.6470116751,0.9199733032,-5.1545188679$

$\mathrm{H}, 0,-2.3261968788,-0.003707411,-4.2449570032$

$\mathrm{H}, 0,-4.6923481648,-0.9253560903,-2.2262928686$

C, $0,-1.545862564,-0.0044026837,4.1993051709$

C, $0,-4.1394605606,-0.0074701207,-2.0125231856$

C, $0,0.531237058,0.0028355347,-4.5719738679$

C, $0,0.9439112381,-0.0004348566,3.1592501605$

C, $0,2.1551813439,0.0021210185,2.4957696505$

C, $0,2.9415950043,0.0044098092,1.4005688415$

C, $0,3.1836308843,0.0060002443,0.0410889928$

C, $0,4.3715371774,0.0073041596,0.9565684269$

C, $0,2.1885136844,0.0005218253,3.9939952115$

$\mathrm{H}, 0,4.9557842251,0.9243789245,1.0581318339$

$\mathrm{H}, 0,4.9590097075,-0.9078904821,1.0564656367$

$\mathrm{H}, 0,2.4727944794,0.9163439976,4.5165065147$

$\mathrm{H}, 0,2.4755211291,-0.9156603076,4.5143797743$

C, $0,-4.1084742084,-0.0070523539,1.5662125221$

$\mathrm{H}, 0,-4.6681512581,0.9085394656,1.7696958518$

$\mathrm{H}, 0,-4.6656501335,-0.9245211367,1.7681095178$

$\mathrm{H}, 0,4.0002152939,0.9268663834,-2.9810040867$

$\mathrm{E}\left(\mathrm{B} 3 \mathrm{LYP} / 6-31 \mathrm{G}^{*}\right.$ opt $)=$

$-846.605585$

$-846.322908$

$-846.305036$

Sum of electronic and thermal Energies=

$-846.304092$

Sum of electronic and thermal Enthalpies=

$-846.367965$

Sum of electronic and thermal Free Energies=

$\mathrm{E}\left(\mathrm{B} 3 \mathrm{LYP} / 6-311+\mathrm{G}^{* *} / / \mathrm{B} 3 \mathrm{LYP} / 6-31 \mathrm{G}^{*}\right)=$

$-846.794885$

93. Heptacyclopropa[15] annulenium cation, non-planar (61a)

C, $0,-3.0408512117,-0.4353639326,-0.888514202$

C, $0,-3.06191022,-0.1790903652,0.4436915276$

C, $0,-2.5999901366,0.1613015061,1.6960264491$

C, $0,-1.6604404636,0.3772451897,2.6439231515$

C, $0,-0.3853651669,0.379720946,3.1724554165$

C, $0,2.8272998898,-0.1614155356,-1.2819784839$

C, $0,2.0188117724,0.1782394873,-2.3443290406$

C, $0,0.8837249359,0.4342841234,-3.0421361556$ 
C, $0,-0.4547254603,0.3722672255,-3.3440780147$

C, $0,-1.7837840351,-0.000388785,-3.2504077192$

C, $0,-2.576636225,-0.3731389355,-2.1796747045$

$\mathrm{H}, 0,-4.8021414695,-0.1022842223,-2.2635916006$

C,0,3.4903179904,0.0392881998,-2.6097004299

$\mathrm{H}, 0,3.8201486944,-0.8252592686,-3.1897619384$

$\mathrm{H}, 0,-1.6741085387,1.664202875,4.468526455$

$\mathrm{H}, 0,-1.8184289117,-0.126484334,4.828829115$

$\mathrm{H}, 0,0.6697563967,0.100902255,-5.2663384154$

$\mathrm{H}, 0,0.4932782018,1.8437065815,-4.7242840789$

$\mathrm{H}, 0,-2.3078061878,-0.0004186944,-4.2053861345$

$\mathrm{H}, 0,-4.2496756154,-1.8449604431,-2.1215573355$

C, $0,-1.5204160552,0.6429287219,4.1134974541$

C, $0,-4.0000699244,-0.7900981885,-1.9855276775$

C, $0,0.4736831958,0.7888882521,-4.4405316458$

C, $0,0.94376315,0.1546198835,3.1179295118$

C, $0,2.123176459,-0.1536360653,2.4709129333$

C, $0,2.88327187,-0.3786693373,1.3791986663$

C, $0,3.1223559307,-0.3765462764,0.0197834495$

C, $0,4.2868379931,-0.6413738856,0.927324896$

C, $0,2.1736149705,0.0007358175,3.9611617185$

$\mathrm{H}, 0,5.0499049274,0.1284764956,1.059865226$

$\mathrm{H}, 0,4.6693781395,-1.662430265,0.988772824$

$\mathrm{H}, 0,2.6548722187,0.8892219589,4.3753583331$

$\mathrm{H}, 0,2.2645196213,-0.8875733084,4.5898252554$

C, $0,-4.0760201027,-0.0399397286,1.5424480306$

$\mathrm{H}, 0,-4.7427998616,0.8243359173,1.5087562755$

$\mathrm{H}, 0,-4.5262812716,-0.9343752467,1.978224473$

$\mathrm{H}, 0,4.0994887202,0.9337897967,-2.7560892407$

$\mathrm{E}\left(\mathrm{B} 3 \mathrm{LYP} / 6-31 \mathrm{G}^{*}\right.$ opt $)=$

$-846.605858$

Sum of electronic and zero-point Energies=

Sum of electronic and thermal Energies=

Sum of electronic and thermal Enthalpies=

Sum of electronic and thermal Free Energies=

$\mathrm{E}\left(\mathrm{B} 3 \mathrm{LYP} / 6-311+\mathrm{G}^{* *} / / \mathrm{B} 3 \mathrm{LYP} / 6-31 \mathrm{G}^{*}\right)=$

$-846.323107$

$-846.303414$

$-846.302470$

$-846.373386$

$-846.795290$

94. 1;2,6;7-Bisdehydro[11] annulenium cation (62)

C, $0,2.0988613557,-1.5696829596,-0.0558759306$

C, $0,2.8897329686,-0.4104114003,-0.0459707622$

C, $0,2.4536990105,0.9231978187,-0.0166337781$

C, $0,1.1416087074,1.3129061483,0.0073054443$

C, $0,0.7300670708,-1.5784603051,-0.0375354206$

C, $0,-0.5019583247,-1.4831812003,-0.0192344002$

C, $0,-0.0682980783,1.5638671812,0.0280837939$

C, $0,-1.3962216812,1.9019131904,0.0520376831$

C, $0,-1.8715522559,-1.4378731297,-0.0001183983$

C, $0,-2.7196728203,-0.3272363801,0.0310759787$

C, $0,-2.520585259,1.0714897054,0.0529349864$

$\mathrm{H}, 0,2.6140341492,-2.5287957273,-0.0797730532$

$\mathrm{H}, 0,3.9651382338,-0.5631880939,-0.0630273228$

$\mathrm{H}, 0,3.2160790043,1.7007790344,-0.0129406614$

$\mathrm{H}, 0,-1.6087598113,2.9710296067,0.0735771679$

$\mathrm{H}, 0,-2.3739987877,-2.4053971062,-0.0104589303$

$\mathrm{H}, 0,-3.7719605266,-0.6016978383,0.0406461155$ 
$\mathrm{H}, 0,-3.4546164241,1.6280981115,0.0755615052$

$\mathrm{E}\left(\mathrm{B} 3 \mathrm{LYP} / 6-31 \mathrm{G}^{*}\right.$ opt $)=$

Sum of electronic and zero-point Energies=

Sum of electronic and thermal Energies=

Sum of electronic and thermal Enthalpies=

Sum of electronic and thermal Free Energies=

$\mathrm{E}\left(\mathrm{B} 3 \mathrm{LYP} / 6-311+\mathrm{G}^{* *} / / \mathrm{B} 3 \mathrm{LYP} / 6-31 \mathrm{G}^{*}\right)=$
$-422.975551$

$-422.836965$

$-422.828000$

$-422.827056$

$-422.870838$

$-423.074205$

95. Dicyclopropa[1,2:4,5][11]annulenium cation, non-planar (63)

C, $0,0.5105695367,-1.2026207559,-1.8240895605$

C, $0,0.4214434032,-0.3291661845,2.4511678608$

C, $0,1.5884873254,-0.6794315011,1.7020048852$

$\mathrm{C}, 0,-1.6719289698,0.1436112362,-1.1512115131$

C, $0,-0.6581222825,1.8912197898,1.5767584772$

$\mathrm{C}, 0,-1.2042551118,2.1719618311,0.3485355071$

C, $0,-1.8864722948,1.3851198673,-0.6320275611$

$\mathrm{C}, 0,-0.4571458279,0.724917094,2.4003559662$

C, $0,-0.7945908784,-0.7976960063,-1.6364190023$

C, $0,1.8900412667,-0.655586964,0.3733162886$

C, $0,1.5114983861,-0.8916828701,-0.9274894204$

C, $0,2.9510506798,-1.0874129466,-0.6012564755$

$\mathrm{H}, 0,2.3879694247,-1.076944449,2.3300654914$

$\mathrm{H}, 0,0.370218941,-0.8745290664,3.3921168076$

$\mathrm{H}, 0,-2.7367433121,1.9083183968,-1.0735656485$

$\mathrm{H}, 0,-0.9978886534,0.846697384,3.3426826244$

$\mathrm{H}, 0,-1.3728645696,3.2367873445,0.1965014484$

$\mathrm{H}, 0,-0.553918532,2.7913243015,2.1884874416$

C, $0,-2.1568811295,-0.7780453228,-2.2365702031$

$\mathrm{H}, 0,-2.844158141,-1.5753356222,-1.9437956152$

$\mathrm{H}, 0,-2.2959324758,-0.4250007712,-3.2606610559$

$\mathrm{H}, 0,0.7590667511,-1.7839483324,-2.7092342051$

$\mathrm{H}, 0,3.3711718473,-2.0951018839,-0.5730735308$

H,0,3.6509141007,-0.3233909045,-0.9479752514

$\mathrm{E}\left(\mathrm{B} 3 \mathrm{LYP} / 6-31 \mathrm{G}^{*} \mathrm{opt}\right)=$

$-501.533348$

Sum of electronic and zero-point Energies=

Sum of electronic and thermal Energies=

Sum of electronic and thermal Enthalpies=

Sum of electronic and thermal Free Energies=

$-501.339790$

$-501.328309$

$-501.327365$

$-501.377542$

$\mathrm{E}\left(\mathrm{B} 3 \mathrm{LYP} / 6-311+\mathrm{G}^{* *} / / \mathrm{B} 3 \mathrm{LYP} / 6-31 \mathrm{G}^{*}\right)=$

$-501.649678$

96. Dicyclopropa[1,2:4,5][11] annulenium cation triplet, non-planar (63t)

C, $0,0.5210724216,-0.6298645097,-1.7769078554$

C, $0,0.6829027851,-0.7424146849,2.3316577321$

C, $0,1.9398604376,-0.640509691,1.7273372536$

C, $0,-1.931414618,0.1249212705,-0.9587643235$

C, $0,-0.4674374629,1.2219718692,1.3729638098$

C, $0,-1.5872213077,1.8355306366,0.7521505245$

C, $0,-2.3907229003,1.185505676,-0.1898583103$

C, $0,-0.4731642666,-0.0850012341,1.8345701615$

C, $0,-0.8398805687,-0.48072631,-1.5557944651$

C, $0,2.0999137492,-0.3285953554,0.3841657564$

C, $0,1.5562302084,-0.3851777036,-0.8870180582$ 
C, $0,3.0467131019,-0.382242364,-0.7635934227$

$\mathrm{H}, 0,2.8227557091,-0.9602321136,2.2770798863$

H, $0,0.5798924427,-1.3970269264,3.1960841882$

$\mathrm{H}, 0,-3.3595377064,1.609088869,-0.4466045696$

$\mathrm{H}, 0,-1.432561193,-0.5902185672,1.9371517248$

$\mathrm{H}, 0,-1.8049399782,2.877828938,0.9814421365$

$\mathrm{H}, 0,0.3928152934,1.847036765,1.608483802$

C, $0,-2.1790413739,-0.6665747803,-2.19523989$

H, $0,-2.6504137076,-1.6505625888,-2.1040842026$

$\mathrm{H}, 0,-2.4257305813,-0.1558374201,-3.13107868$

$\mathrm{H}, 0,0.8112279757,-0.9806001232,-2.7663816368$

$\mathrm{H}, 0,3.6237443797,-1.2922902644,-0.9542496642$

$\mathrm{H}, 0,3.5758861315,0.5318765145,-1.0518564605$

$\mathrm{E}\left(\mathrm{UB} 3 \mathrm{LYP} / 6-31 \mathrm{G}^{*}\right.$ opt $)=$

$-501.523459$

$-501.332018$

$-501.320818$

$-501.319874$

$-501.369352$

$-501.638675$

97. Dicyclopropa[1,2:3,4][11] annulenium cation, non-planar (64)

C, $0,0.5780252954,-1.0876181604,-1.1135722818$

C, $0,0.5941053993,-0.4257784659,1.8779299062$

C, $0,1.9456108987,-0.0329214183,1.6238588924$

C, $0,-1.6803692637,0.3190180057,-0.9139963074$

C,0,-0.6629676142,1.7443466203,1.6414241871

C, $0,-1.5614311601,2.238557008,0.7203275957$

C, $0,-2.1426297605,1.4871348845,-0.3383523425$

C, $0,-0.5423027069,0.3445896585,1.864699884$

C, $0,-0.6814118947,-0.5781246427,-1.2463555593$

C,0,2.5942505506,-0.5954232096,0.5352273123

C, $0,1.8097984849,-1.0908222309,-0.509755223$

C, $0,1.6991494357,-1.8240551774,-1.8044452039$

$\mathrm{H}, 0,2.4526377147,0.705381762,2.2452049679$

$\mathrm{H}, 0,0.4111310239,-1.4954415906,1.7993442216$

$\mathrm{H}, 0,-2.9903520279,1.9455876183,-0.8458384537$

$\mathrm{H}, 0,2.1207938868,-1.3884407197,-2.7130143601$

$\mathrm{H}, 0,1.7411708784,-2.9155883898,-1.779804829$

$\mathrm{H}, 0,-1.4712589029,-0.2189813222,1.7974808804$

H, $0,3.6740162919,-0.5460957897,0.4191432524$

$\mathrm{H}, 0,-1.7882914104,3.3028983572,0.7303368365$

$\mathrm{H}, 0,-0.021024277,2.4411722095,2.1801351861$

C, $0,-1.8980736398,-0.5526497951,-2.1170012418$

$\mathrm{H}, 0,-2.5971650125,-1.3880624715,-2.0346479385$

H,0,-1.8421823136,-0.1199481226,-3.118277472

$\mathrm{E}(\mathrm{B} 3 \mathrm{LYP} / 6-31 \mathrm{G} *$ opt $)=$

$-501.537923$

Sum of electronic and zero-point Energies=

Sum of electronic and thermal Energies=

$-501.344591$

$-501.333598$

$-501.332654$

$-501.380693$

Sum of electronic and thermal Free Energies=

$-501.653359$

98. Dicyclopropa[1,2:3,4][11]annulenium cation triplet, non-planar (64t) 
C, $0,0.3386333429,-0.6850819289,-1.3275372542$

C, $0,0.2848298118,-1.0608403823,2.2689953554$

C, $0,1.5761478299,-1.4540074358,1.8550903135$

C,0,-1.6644066441,0.9623455962,-0.9213139901

C, $0,0.0822621471,1.2616801936,1.4153322655$

C,0,-0.6937866728,2.3128099204,0.8485806661

C, $0,-1.6624266039,2.1135885265,-0.1323592614$

C, $0,-0.4576585076,0.0336024324,1.785915061$

C, $0,-0.8634412886,-0.0482171489,-1.401709345$

C,0,2.1217970171,-1.3713074966,0.5704328896

C, $0,1.4527712302,-1.0406773719,-0.616099859$

C, $0,1.4671463951,-1.3274822584,-2.0848323844$

$\mathrm{H}, 0,2.1563693225,-2.0369204174,2.5690686663$

H, $0,-0.1900077423,-1.685344606,3.0262158757$

$\mathrm{H}, 0,-2.330504958,2.9276372321,-0.4026544054$

$\mathrm{H}, 0,2.0334663576,-0.6886856386,-2.7671916786$

$\mathrm{H}, 0,1.4096131007,-2.3713297159,-2.4048852168$

$\mathrm{H}, 0,-1.5422651535,-0.0350891423,1.8545579297$

$\mathrm{H}, 0,3.1238446927,-1.774087615,0.4424541568$

$\mathrm{H}, 0,-0.4772825769,3.3353273705,1.155135328$

$\mathrm{H}, 0,1.1156847992,1.4763584192,1.684066802$

C, $0,-2.0581099396,0.3503936613,-2.2215841199$

$\mathrm{H}, 0,-2.8985130501,-0.3491877214,-2.2425326437$

$\mathrm{H}, 0,-1.9429534972,0.92048399,-3.1476968358$

$\mathrm{E}\left(\mathrm{UB} 3 \mathrm{LYP} / 6-31 \mathrm{G}^{*}\right.$ opt $)=$

$-501.519096$

Sum of electronic and zero-point Energies $=\quad-501.327283$

Sum of electronic and thermal Energies= $\quad-501.316061$

Sum of electronic and thermal Enthalpies $=\quad-501.315116$

Sum of electronic and thermal Free Energies $=\quad-501.364661$

$\mathrm{E}\left(\mathrm{UB} 3 \mathrm{LYP} / 6-311+\mathrm{G}^{* *} / / \mathrm{UB} 3 \mathrm{LYP} / 6-31 \mathrm{G}^{*}\right)=\quad-501.634504$

99. 1,2-Dihydrobenzene, held planar (NImag=1)

$\begin{array}{lrrr}6 & 0.138472 & -1.430314 & 0.000044 \\ 6 & 1.282648 & -0.733467 & -0.000262 \\ 6 & 1.282535 & 0.733625 & 0.000269 \\ 6 & 0.138288 & 1.430344 & -0.000056 \\ 6 & -1.225492 & 0.780379 & -0.000226 \\ 6 & -1.225380 & -0.780537 & 0.000231 \\ 1 & 0.157807 & -2.518850 & -0.000160 \\ 1 & 2.239998 & -1.249465 & -0.000525 \\ 1 & 2.239813 & 1.249770 & 0.000529 \\ 1 & 0.157484 & 2.518878 & 0.000131 \\ 1 & -1.785235 & 1.152289 & -0.870306 \\ 1 & -1.785542 & -1.153063 & -0.869311 \\ 1 & -1.785687 & 1.152803 & 0.869330 \\ 1 & -1.785066 & -1.152547 & 0.870311\end{array}$

$\mathrm{E}\left(\mathrm{B} 3 \mathrm{LYP} / 6-31 \mathrm{G}^{*}\right.$ opt $)=$

$-233.415885$

Sum of electronic and zero-point Energies $=\quad-233.293215$

Sum of electronic and thermal Energies $=\quad-233.288620$

Sum of electronic and thermal Enthalpies $=\quad-233.287676$

Sum of electronic and thermal Free Energies $=\quad-233.321026$

$\mathrm{E}\left(\mathrm{B} 3 \mathrm{LYP} / 6-311+\mathrm{G}^{* *} / / \mathrm{B} 3 \mathrm{LYP} / 6-31 \mathrm{G}^{*}\right)=\quad-233.480334$ 
$\chi=-41.9$

100. 1,2-Dihydrotropylium cation, held planar (NImag=1)

$\begin{array}{lrcc}6 & -1.471529 & 0.774262 & 0.000027 \\ 6 & -0.237615 & 1.602164 & -0.000006 \\ 6 & 1.095917 & 1.288627 & -0.000018 \\ 6 & -1.469026 & -0.778475 & -0.000002 \\ 6 & 1.677304 & 0.002458 & -0.000002 \\ 6 & -0.232795 & -1.602938 & -0.000066 \\ 6 & 1.099819 & -1.285394 & 0.000030 \\ 1 & -2.056340 & 1.138100 & -0.859216 \\ 1 & -0.461406 & 2.669530 & 0.000098 \\ 1 & 1.794160 & 2.120446 & -0.000017 \\ 1 & -2.053284 & -1.144277 & -0.858781 \\ 1 & 2.766922 & 0.004076 & 0.000030 \\ 1 & -0.453503 & -2.670924 & -0.000120 \\ 1 & 1.800473 & -2.115182 & 0.000091 \\ 1 & -2.052737 & -1.144195 & 0.859223 \\ 1 & -2.056743 & 1.138199 & 0.858916\end{array}$

$\mathrm{E}\left(\mathrm{B} 3 \mathrm{LYP} / 6-31 \mathrm{G}^{*}\right.$ opt $)=$

$-271.847627$

Sum of electronic and zero-point Energies $=\quad-271.707248$

Sum of electronic and thermal Energies $=\quad-271.701541$

Sum of electronic and thermal Enthalpies $=\quad-271.700596$

Sum of electronic and thermal Free Energies $=\quad-271.736796$

$\mathrm{E}\left(\mathrm{B} 3 \mathrm{LYP} / 6-311+\mathrm{G}^{* *} / / \mathrm{B} 3 \mathrm{LYP} / 6-31 \mathrm{G}^{*}\right)=\quad-271.912422$

$\chi=-33.9$

101. 1,2-Dihydro[11]annulenium cation, all cis, held planar (NImag=4)

$\begin{array}{rrrr}6 & -1.453260 & 2.116853 & 0.000000 \\ 6 & 0.000000 & 2.489096 & 0.000000 \\ 6 & 1.326574 & 2.157420 & 0.000000 \\ 6 & 2.293049 & 1.095967 & 0.000000 \\ 6 & 2.569045 & -0.269741 & 0.000000 \\ 6 & 2.033644 & -1.576753 & 0.000000 \\ 6 & 0.900988 & -2.420557 & 0.000000 \\ 6 & -0.490401 & -2.493359 & 0.000000 \\ 6 & -1.759294 & -1.822014 & 0.000000 \\ 6 & -2.411081 & -0.619901 & 0.000000 \\ 6 & -2.412520 & 0.880280 & 0.000000 \\ 1 & 1.897483 & 3.082761 & 0.000000 \\ 1 & 3.260372 & 1.595742 & 0.000000 \\ 1 & 3.650635 & -0.377277 & 0.000000 \\ 1 & 2.894083 & -2.244045 & 0.000000 \\ 1 & 1.274385 & -3.441342 & 0.000000 \\ 1 & -0.733371 & -3.554723 & 0.000000 \\ 1 & -2.513187 & -2.605470 & 0.000000 \\ 1 & -3.480302 & -0.840379 & 0.000000 \\ 1 & -3.084437 & 1.070289 & 0.851030 \\ 1 & -1.804455 & 2.720442 & -0.850901 \\ 1 & -1.804455 & 2.720442 & 0.850901 \\ 1 & -3.084437 & 1.070289 & -0.851030 \\ 1 & -0.052783 & 3.579529 & 0.000000\end{array}$


$\mathrm{E}\left(\mathrm{B} 3 \mathrm{LYP} / 6-31 \mathrm{G}^{*}\right.$ opt $)=$

Sum of electronic and zero-point Energies= Sum of electronic and thermal Energies= Sum of electronic and thermal Enthalpies= Sum of electronic and thermal Free Energies= $\mathrm{E}\left(\mathrm{B} 3 \mathrm{LYP} / 6-311+\mathrm{G}^{* *} / / \mathrm{B} 3 \mathrm{LYP} / 6-31 \mathrm{G}^{*}\right)=$ $\chi=-26.9$
$-426.519348$

$-426.309711$

$-426.302012$

$-426.301068$

$-426.342658$

$-426.625177$

102. [11]Annulenium, di-trans, 5+4 type-indene cation, isomer of $\mathbf{3 5}$<smiles></smiles>

C, $0,-1.7828830245,-0.0367103818,-1.0562074625$

C, $0,-1.8162141104,0.0441342072,0.371121183$

C, $0,-0.8625312927,0.019355979,1.4602621453$

C, $0,0.5066305362,-0.2340088833,1.5518055481$

C, $0,-0.8039961513,0.0963819297,-2.0375333392$

C, $0,0.5286712424,0.4828090077,-1.8279599983$

C, $0,1.3353964538,-0.6791032131,0.515259436$

C, $0,2.6714509356,-0.3339072968,0.4034237675$

C, $0,1.5817941866,0.0228912393,-2.5973058826$

C, $0,2.9168823366,-0.1508516145,-2.1287654595$

C, $0,3.3923852654,-0.2382814119,-0.8223013288$

$\mathrm{H}, 0,-2.7654462541,-0.2711967379,-1.4632061678$

$\mathrm{H}, 0,1.3788284658,-0.3163390175,-3.6133664191$

$\mathrm{H}, 0,0.7568893393,0.9994667703,-0.9053185876$

$\mathrm{H}, 0,-1.0861575491,-0.2386675089,-3.0349939979$

$\mathrm{H}, 0,0.979625966,0.0470932463,2.4934021866$

$\mathrm{H}, 0,0.8584177489,-1.1552801637,-0.329255684$

$\mathrm{H}, 0,3.6706745032,-0.2757888792,-2.9043990582$

$\mathrm{H}, 0,4.4754580799,-0.1940322293,-0.7212956484$

$\mathrm{H}, 0,3.2097885929,-0.0337170598,1.3027160047$

C, $0,-3.1364016418,0.2124040613,0.976142928$

C, $0,-1.6215220826,0.3456651927,2.7366182928$

C, $0,-3.0473948311,0.4014307543,2.3090175253$

$\mathrm{H}, 0,-3.8733022374,0.5900831357,2.9848902652$

$\mathrm{H}, 0,-1.2891554008,1.3075290704,3.1581785259$

H,0,-1.4404133968,-0.4035771826,3.520659529

$\mathrm{H}, 0,-4.0488147911,0.2311691385,0.3905249204$

$\mathrm{E}\left(\mathrm{B} 3 \mathrm{LYP} / 6-31 \mathrm{G}^{*} \mathrm{opt}\right)=$

$-540.979404$

Sum of electronic and zero-point Energies=

Sum of electronic and thermal Energies=

$-540.753113$

$-540.741811$

Sum of electronic and thermal Enthalpies=

$-540.740867$

Sum of electronic and thermal Free Energies=

$-540.789719$

$\mathrm{E}\left(\mathrm{B} 3 \mathrm{LYP} / 6-311+\mathrm{G}^{* *} / / \mathrm{B} 3 \mathrm{LYP} / 6-31 \mathrm{G}^{*}\right)=$

$-541.106656$

$\chi=-122.2$

103. [11]Annulenium, di-trans, 5+4 type-indene cation, another isomer of $\mathbf{3 5}$ 
<smiles>C/C=C/C=C\C=C/C=C/C=C1/C=CCC1</smiles>

C,0,-1.771238653,0.0163977663,-1.0379976837 C, $0,-1.8126686897,0.0471613605,0.3626931538$ C, $0,-0.8803249735,0.0461011207,1.4724268034$ C, $0,0.5310737931,-0.1138110593,1.5643328104$ C, $0,-0.758967813,0.064172472,-2.0283454469$ C, $0,0.5540169812,0.4372274857,-1.8240240211$ C, $0,1.3504238048,-0.5893609108,0.5667591499$ C, $0,2.7242078655,-0.2964880728,0.4449088131$ C, $0,1.6416629858,-0.0074191712,-2.5995547575$ C, $0,2.9322587893,-0.211729545,-2.1042553813$ C,0,3.4133197753,-0.2065410655,-0.767037038 $\mathrm{H}, 0,-2.7626707467,-0.1352114569,-1.4663440649$ $\mathrm{H}, 0,1.4608918778,-0.2897302694,-3.6360657876$ H, $0,0.7850733777,0.9737408089,-0.9136367042$ H,0,-1.0412049086,-0.3349438915,-3.0019687876 $\mathrm{H}, 0,0.9888424796,0.2774648957,2.4723247205$ $\mathrm{H}, 0,0.8809361999,-1.0986212719,-0.2638098935$ H,0,3.6924295791,-0.4280323807,-2.8530302843 $\mathrm{H}, 0,4.493882302,-0.1036811117,-0.6816344848$ $\mathrm{H}, 0,3.2827901995,-0.0487163643,1.3466194537$ C, $0,-3.2068115341,0.1586880415,0.9764966804$ C, $0,-1.635684124,0.309081416,2.6859745378$ C,0,-2.9619799572,0.3587268102,2.4297677855 $\mathrm{H}, 0,-3.7497727871,0.4975044073,3.161321571$ $\mathrm{H}, 0,-3.790372742,-0.7571392788,0.7915631591$ $\mathrm{H}, 0,-1.1686526931,0.3952906938,3.6608607543$ $\mathrm{H}, 0,-3.7879016402,0.97883533,0.5309279139$

$\mathrm{E}\left(\mathrm{B} 3 \mathrm{LYP} / 6-31 \mathrm{G}^{*}\right.$ opt $)=$ Sum of electronic and zero-point Energies= Sum of electronic and thermal Energies= Sum of electronic and thermal Enthalpies= Sum of electronic and thermal Free Energies= $\mathrm{E}\left(\mathrm{B} 3 \mathrm{LYP} / 6-311+\mathrm{G}^{* *} / / \mathrm{B} 3 \mathrm{LYP} / 6-31 \mathrm{G}^{*}\right)=$ $\chi=-122.7$
$-540.980982$

$-540.754544$

$-540.743286$

$-540.742342$

$-540.791100$

$-541.108198$

104. [11]Annulenium, di-trans, 5+4 type-isoindene cation, isomer of $\mathbf{3 6}$<smiles></smiles>

C, $0,-1.79842217,0.0248437871,-1.0757680832$

C, $0,-1.8553325997,-0.0494246331,0.3666340279$

$\mathrm{C}, 0,-0.8754078821,-0.0632149643,1.5217361232$

$\mathrm{C}, 0,0.5848391341,-0.0845791446,1.5283596458$

$\mathrm{C}, 0,-0.8087241912,0.2075667391,-2.0317374257$

C, $0,0.5333708196,0.5262326301,-1.7821586681$

C, $0,1.3775639092,-0.5838602294,0.5530662363$

C,0,2.7612036529,-0.19838978,0.398305502 
C, $0,1.6182474877,0.2045857954,-2.6115167609$

C, $0,2.9177584112,0.0572880129,-2.1328344877$

C, $0,3.4174065276,0.0497242972,-0.7883583513$

$\mathrm{H}, 0,-2.7756889823,-0.1746762048,-1.5128901701$

$\mathrm{H}, 0,1.4328592448,-0.0493754732,-3.6532720509$

$\mathrm{H}, 0,0.7620374822,1.0045905665,-0.8415983583$

$\mathrm{H}, 0,-1.092213429,-0.02986123,-3.0556748041$

$\mathrm{H}, 0,1.051019601,0.4343414774,2.3656484453$

$\mathrm{H}, 0,0.9248515271,-1.1386668332,-0.2631268338$

$\mathrm{H}, 0,3.6801343042,-0.1116591785,-2.8928153677$

$\mathrm{H}, 0,4.4873598551,0.2303241231,-0.710365188$

$\mathrm{H}, 0,3.3398721782,-0.0526559571,1.3102883614$

C, $0,-1.5955650738,-0.0113774503,2.6734760359$

C, $0,-3.1201829724,-0.0648828775,0.9150782503$

C, $0,-3.0621427307,-0.0192991931,2.3970045844$

$\mathrm{H}, 0,-1.1726120272,-0.0031104201,3.6714827093$

$\mathrm{H}, 0,-4.0432776977,-0.0771544165,0.3459536136$

$\mathrm{H}, 0,-3.5839006755,-0.8747248723,2.8560646652$

$\mathrm{H}, 0,-3.5781153151,0.8713504811,2.7925852053$

$\mathrm{E}\left(\mathrm{B} 3 \mathrm{LYP} / 6-31 \mathrm{G}^{*}\right.$ opt $)=$

Sum of electronic and zero-point Energies=

Sum of electronic and thermal Energies=

Sum of electronic and thermal Enthalpies=

Sum of electronic and thermal Free Energies=

$\mathrm{E}\left(\mathrm{B} 3 \mathrm{LYP} / 6-311+\mathrm{G}^{* *} / / \mathrm{B} 3 \mathrm{LYP} / 6-31 \mathrm{G}^{*}\right)=$

$\chi=-70.8$
$-540.957659$

$-540.732312$

$-540.720819$

$-540.719875$

$-540.769174$

$-541.085665$

105. [11]Annulenium, all cis-indene cation, another isomer of $\mathbf{4 5}$<smiles>[C+]1C=C[C@H]2CC=CC3=C1CC=C[C@@H]32</smiles>

C, $0,0.201803462,-0.4540809039,-1.7051427626$

C, $0,0.3744179126,-0.1198949426,2.7489957713$

C, $0,1.3055623165,-0.7154868201,1.9134026435$

C, $0,1.2145426828,1.7696710932,-1.3058994808$

C, $0,-1.3166262486,1.4215736512,1.5177287505$

C, $0,-0.7216143355,1.6860159627,0.2394199318$

C, $0,0.5901851907,1.9635166018,-0.0197087547$

C, $0,-0.8136937473,0.6762581528,2.5599787178$

C, $0,0.9216577127,0.7101668416,-2.1372552256$

C, $0,1.3183281696,-0.7712382749,0.4992687144$

C, $0,0.2765616946,-0.9922552134,-0.3838006589$

$\mathrm{H}, 0,2.2864196148,-0.5456231731,0.0458062077$

$\mathrm{H}, 0,2.2283268212,-1.019715137,2.4069563344$

$\mathrm{H}, 0,0.6074889721,-0.2442473822,3.8067527547$

$\mathrm{H}, 0,-1.4335151763,0.6952059491,3.4561331411$

$\mathrm{H}, 0,-2.3354498305,1.7839003676,1.6533393452$

$\mathrm{H}, 0,-1.4032345865,1.6300897257,-0.6110812043$

$\mathrm{H}, 0,1.2236211085,2.3146600624,0.7950310462$

$\mathrm{H}, 0,1.946231642,2.5036434916,-1.6427558019$

$\mathrm{H}, 0,1.2333565322,0.7591904601,-3.1798741323$

C, $0,-0.9092220871,-1.9162121606,-0.2079632575$ 
C, $0,-0.7916877835,-1.192735524,-2.452420764$

C, $0,-1.4421276951,-2.0338446199,-1.6074436562$

$\mathrm{H}, 0,-0.6495380807,-2.8820842896,0.2411841771$

$\mathrm{H}, 0,-2.2648590896,-2.6879231581,-1.8748058267$

$\mathrm{H}, 0,-1.0186023978,-1.0319873503,-3.4999499941$

$\mathrm{H}, 0,-1.6687689948,-1.4638326301,0.4483041386$

$\mathrm{E}(\mathrm{B} 3 \mathrm{LYP} / 6-31 \mathrm{G} *$ opt $)=$

$-540.954036$

Sum of electronic and zero-point Energies=

$-540.729832$

Sum of electronic and thermal Energies=

$-540.718215$

Sum of electronic and thermal Enthalpies=

$-540.717271$

Sum of electronic and thermal Free Energies=

$-540.766671$

$\mathrm{E}\left(\mathrm{B} 3 \mathrm{LYP} / 6-311+\mathrm{G}^{* *} / / \mathrm{B} 3 \mathrm{LYP} / 6-31 \mathrm{G}^{*}\right)=$

$\chi=-95.6$

$-541.082722$

106. [11]Annulenium, all cis-indene cation, another isomer of $\mathbf{4 5}$

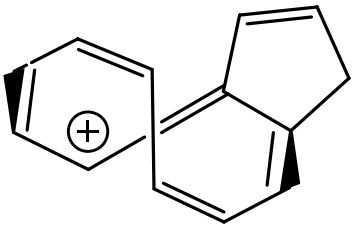

$\mathrm{C}, 0,-0.6331791467,-1.5649990256,-1.7414747301$

C, $, 0,-0.1772075834,-0.9127277703,2.572422378$

C, $0,0.8288689198,-1.3959339374,1.6832007369$

C, $0,0.4470650426,0.6225682156,-1.5795883157$

$\mathrm{C}, 0,-2.034651142,0.3233246409,1.2472262282$

C, $, 0,-1.53585005,0.6906433395,0.0219642077$

C, $, 0,-0.1891406947,0.9970691068,-0.3491606694$

C, $0,-1.3856073277,-0.2532848731,2.3830966543$

C, $0,0.0996175331,-0.4926562138,-2.3271386542$

C, $0,0.7418990999,-1.6794963611,0.3400969211$

C, $0,-0.4454722891,-1.9257659082,-0.41662632$

$\mathrm{H}, 0,0.3981928136,-0.5540317384,-3.3745055$

$\mathrm{H}, 0,-1.3465939952,-2.1165927979,-2.3525351874$

$\mathrm{H}, 0,-1.2394916781,-2.4860045036,0.0784289474$

$\mathrm{H}, 0,1.6803753264,-1.7599807815,-0.2060013832$

$\mathrm{H}, 0,1.8119362597,-1.5108161126,2.1380354026$

$\mathrm{H}, 0,0.065569883,-1.0721126657,3.6228962501$

$\mathrm{H}, 0,-1.9861164704,-0.1881948085,3.2900854324$

$\mathrm{H}, 0,-3.1166818264,0.3961113131,1.3523474088$

$\mathrm{H}, 0,-2.2583727914,0.7670486232,-0.7905576685$

C, $0,0.7058835233,1.8768039879,0.3580015022$

C, $0,1.6442998192,1.5256708633,-1.7833501999$

C, $0,1.7533982154,2.202101273,-0.4501100321$

$\mathrm{H}, 0,0.5041919603,2.2865047014,1.3402399207$

$\mathrm{H}, 0,2.5462295128,2.9000709339,-0.2029829462$

$\mathrm{H}, 0,2.558039405,0.9896106492,-2.070037649$

$\mathrm{H}, 0,1.4631780824,2.2684831629,-2.5767712703$

$\mathrm{E}\left(\mathrm{B} 3 \mathrm{LYP} / 6-31 \mathrm{G}^{*}\right.$ opt $)=$

$-540.957996$

$-540.733688$

$-540.722163$

Sum of electronic and thermal Energies=

$-540.721219$

Sum of electronic and thermal Enthalpies=

$-540.770332$

Sum of electronic and thermal Free Energies=

$-541.087012$ 
$\chi=-101.8$

107. [11]Annulenium, all cis-isoindene cation, isomer of $\mathbf{4 6}$<smiles>c1ccc(-c2cccc3ccccc23)cc1</smiles>

C, $0,-0.5878828958,-1.6145787598,-1.7114446424$

$\mathrm{C}, 0,-0.268508865,-1.2116160023,2.5973858172$

C, $0,0.843403826,-1.4020196253,1.7342668485$

C, $0,0.4486616123,0.6485674613,-1.5484127483$

C, $0,-2.0227560768,0.1617049335,1.2085900236$

C,0,-1.4923709912,0.8619803282,0.1794044707

C, $0,-0.1074584438,1.1520592645,-0.2535199623$

C, $0,-1.47395062,-0.6071753323,2.3222865761$

C, $0,-0.050580874,-0.4829720884,-2.2954220936$

C, $0,0.7724363331,-1.5428811687,0.3587841578$

C, $0,-0.3750972641,-1.9309673687,-0.3508898974$

$\mathrm{H}, 0,-0.0375929488,-0.4153053532,-3.3828747831$

$\mathrm{H}, 0,-1.1911513902,-2.2858533321,-2.318946602$

$\mathrm{H}, 0,-1.102126559,-2.563394467,0.1581119152$

$\mathrm{H}, 0,1.6767051768,-1.3522285522,-0.214361895$

$\mathrm{H}, 0,1.8303543813,-1.3986247159,2.1960744654$

$\mathrm{H}, 0,-0.1222081109,-1.5168196448,3.6323897035$

$\mathrm{H}, 0,-2.1834342716,-0.664293097,3.1478243606$

$\mathrm{H}, 0,-3.1077628687,0.2249007676,1.2737486083$

$\mathrm{H}, 0,-2.2312151385,1.3454204053,-0.4621693826$

C, $0,0.7212042163,2.1117954666,0.2106483751$

C, $0,1.5568372742,1.3859097246,-1.8668439628$

C, $0,1.864065556,2.3281770643,-0.7462569456$

$\mathrm{H}, 0,0.5533335566,2.7247469666,1.0884048371$

$\mathrm{H}, 0,2.8495731028,2.1113736545,-0.3032386005$

$\mathrm{H}, 0,2.1679688347,1.2700591158,-2.7556989623$

H,0,1.9295395121,3.3721148675,-1.090719763

$\mathrm{E}\left(\mathrm{B} 3 \mathrm{LYP} / 6-31 \mathrm{G}^{*}\right.$ opt $)=$

$-540.943531$

$-540.719187$

Sum of electronic and zero-point Energies=

Sum of electronic and thermal Energies=

$-540.707572$

Sum of electronic and thermal Enthalpies=

$-540.706627$

Sum of electronic and thermal Free Energies=

$-540.756012$

$\mathrm{E}\left(\mathrm{B} 3 \mathrm{LYP} / 6-311+\mathrm{G}^{* *} / / \mathrm{B} 3 \mathrm{LYP} / 6-31 \mathrm{G}^{*}\right)=$ $\chi=-84.9$

$-541.072914$

108. [11]Annulenium, all cis-indene cation, another isomer of $\mathbf{4 5}$<smiles>[C-]1C=CC2C=CC3CCCC3C=C12</smiles>

$\mathrm{C}, 0,-0.7116005028,-1.1682520018,-1.3503092741$

C, $0,-0.1738965627,-0.7908524728,2.9798474615$

C, $0,0.734979161,-1.3649685654,2.0432758548$

C, $0,0.5320763216,0.9593385113,-0.8872798119$

C, $0,-2.0073122849,0.5975983434,1.8214988257$ 
C, $0,-1.4576996731,0.9556905654,0.5962946835$

C, $0,-0.1049308727,1.232960419,0.3289076671$

C, $0,-1.3638193116,-0.0574339325,2.8918311159$

C, $0,0.1513708888,-0.0871792929,-1.7684298523$

C, $0,0.6148791166,-1.6128917208,0.6964303677$

C, $0,-0.5801377949,-1.7190816392,-0.1027461085$

$\mathrm{H}, 0,-1.4885390047,-1.5164988528,-2.0292833269$

$\mathrm{H}, 0,-1.4069904308,-2.3020578341,0.3034889559$

$\mathrm{H}, 0,1.5458486488,-1.8022364481,0.1636336995$

$\mathrm{H}, 0,1.7101366113,-1.5884483891,2.4743638881$

$\mathrm{H}, 0,0.1309486492,-0.9666578898,4.011773511$

$\mathrm{H}, 0,-1.9061829964,-0.0041751096,3.8356772365$

$\mathrm{H}, 0,-3.0745788463,0.7713998165,1.9537721479$

$\mathrm{H}, 0,-2.1497629825,1.0392072159,-0.2406342391$

$\mathrm{H}, 0,0.4828414152,1.6939278828,1.124209517$

C, $0,1.654670602,1.729874013,-1.5436679926$

C, $0,0.8299700216,0.093234476,-3.0303207476$

C, $0,1.7088460041,1.1254426938,-2.9147112399$

$\mathrm{H}, 0,2.6109685363,1.5870551649,-1.015616208$

H, $0,0.7044950997,-0.5482712893,-3.8946381318$

$\mathrm{H}, 0,1.4820561201,2.814510759,-1.565493395$

$\mathrm{H}, 0,2.3943885023,1.4613685946,-3.6849793519$

$\mathrm{E}\left(\mathrm{B} 3 \mathrm{LYP} / 6-31 \mathrm{G}^{*}\right.$ opt $)=$

$-540.957174$

Sum of electronic and zero-point Energies=

Sum of electronic and thermal Energies=

Sum of electronic and thermal Enthalpies=

Sum of electronic and thermal Free Energies=

$\mathrm{E}\left(\mathrm{B} 3 \mathrm{LYP} / 6-311+\mathrm{G}^{* *} / / \mathrm{B} 3 \mathrm{LYP} / 6-31 \mathrm{G}^{*}\right)=$

$-540.733070$

$-540.721511$

$-540.720567$

$-540.769909$

$\chi=-101.3$

$-541.086062$

109. [11]Annulenium, all cis-indene cation, another isomer of $\mathbf{4 5}$

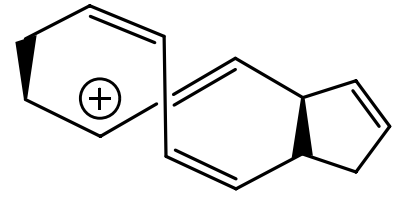

C, $0,-0.482284918,-1.2828493304,-1.3622426668$

C, $0,-0.2749200599,-0.8357775887,3.0954223227$

C, $0,0.6103337944,-1.4770558805,2.251110881$

C, $0,0.5872615296,0.9494884796,-0.9505154412$

C, $0,-1.9223709824,0.7706340675,1.8823643737$

C, $0,-1.3239631141,1.0052638683,0.606563671$

C, $0,-0.0004231996,1.2161246522,0.3329796011$

C, $0,-1.4415537378,-0.0038397877,2.9145423253$

C, $0,0.2120613351,-0.1503482936,-1.7857809706$

C, $0,0.5942723162,-1.5570662334,0.8318593301$

C, $0,-0.4944064865,-1.7008059013,-0.0044504469$

$\mathrm{H}, 0,-1.0356468862,-1.8650747209,-2.1004095123$

$\mathrm{H}, 0,-1.4043973341,-2.152504961,0.3896069259$

$\mathrm{H}, 0,1.5727123448,-1.4823528298,0.3548350497$

$\mathrm{H}, 0,1.5031755717,-1.8695037973,2.7364548601$

$\mathrm{H}, 0,-0.0462067308,-0.9787473398,4.1516533376$

$\mathrm{H}, 0,-2.0671528874,0.0128078994,3.8068142704$

$\mathrm{H}, 0,-2.9337570244,1.1521529932,2.016752132$

$\mathrm{H}, 0,-2.0130905249,0.9618436823,-0.2386512928$ 
$\mathrm{H}, 0,0.6591153849,1.5561539229,1.130762336$

C, $0,1.5433149937,1.7623346378,-1.6672334252$

C, $0,0.7756446678,0.1088191797,-3.1659092814$

C, $0,1.6488889592,1.3076180775,-2.9436685687$

$\mathrm{H}, 0,1.3238732412,-0.7484785068,-3.5789729202$

$\mathrm{H}, 0,2.2484040507,1.7574244196,-3.7274332306$

$\mathrm{H}, 0,2.0233910666,2.6445946417,-1.2602004742$

$\mathrm{H}, 0,-0.0215508583,0.336444914,-3.8914617067$

$\mathrm{E}\left(\mathrm{B} 3 \mathrm{LYP} / 6-31 \mathrm{G}^{*}\right.$ opt $)=$

$-540.956954$

Sum of electronic and zero-point Energies=

$-540.732781$

Sum of electronic and thermal Energies=

Sum of electronic and thermal Enthalpies=

$-540.721181$

Sum of electronic and thermal Free Energies=

$-540.720236$

$\mathrm{E}\left(\mathrm{B} 3 \mathrm{LYP} / 6-311+\mathrm{G}^{* *} / / \mathrm{B} 3 \mathrm{LYP} / 6-31 \mathrm{G}^{*}\right)=$

$-540.769644$

$\chi=-97.0$

$-541.085735$

110. [11]Annulenium, all cis-indene cation, another isomer of $\mathbf{4 6}$

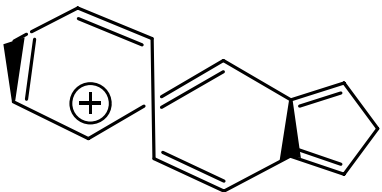

C, $0,-0.0265925717,-1.4697829144,-1.1162855011$

$\mathrm{C}, 0,-0.9932816519,-1.1253476992,3.0483670613$

C, $0,0.2710745251,-1.4431778291,2.6512136068$

C, $0,0.6224176918,1.1172577643,-1.0628018096$

$\mathrm{C}, 0,-1.6073882844,0.598603735,1.372726915$

C, $0,-0.284782956,1.2711916501,1.3503322417$

C, $0,0.5818257633,1.5349676099,0.3463591777$

C, $0,-1.9470770094,-0.4196912173,2.1981737832$

C, $0,0.3386083337,-0.2124982398,-1.6838139762$

C, $0,0.6762998413,-1.2784786168,1.2776091143$

C, $0,-0.0451738517,-1.8433482746,0.2168684599$

$\mathrm{H}, 0,-0.4520135835,-2.1834577609,-1.8203013354$

$\mathrm{H}, 0,-0.708045078,-2.6666016123,0.4749279132$

$\mathrm{H}, 0,1.639731941,-0.8153374725,1.0747856995$

$\mathrm{H}, 0,1.0178192825,-1.7377777288,3.3867579725$

$\mathrm{H}, 0,-1.2868018887,-1.32877929,4.0774540048$

$\mathrm{H}, 0,-2.9955576113,-0.6993732918,2.2824286165$

$\mathrm{H}, 0,-2.3896958457,1.0454576689,0.7596651086$

$\mathrm{H}, 0,-0.0157390774,1.7106327995,2.3123519615$

$\mathrm{H}, 0,1.398849861,2.2049961842,0.611471569$

C, $0,0.9914737763,1.9605183903,-2.0599711323$

C, $0,0.4911353652,-0.0894696399,-3.0479526227$

C, $0,0.9369276786,1.2848103958,-3.3978215947$

$\mathrm{H}, 0,1.2608043848,3.0025561514,-1.9296358225$

$\mathrm{H}, 0,0.3451843617,-0.8890410627,-3.7669923509$

$\mathrm{H}, 0,1.9083178763,1.2704819105,-3.9185127005$

$\mathrm{H}, 0,0.244345476,1.7729128187,-4.1024229765$

$\mathrm{E}\left(\mathrm{B} 3 \mathrm{LYP} / 6-31 \mathrm{G}^{*} \mathrm{opt}\right)=$

$-540.939813$

Sum of electronic and zero-point Energies=

Sum of electronic and thermal Energies=

$-540.715984$

$-540.704374$

Sum of electronic and thermal Enthalpies=

$-540.703430$

Sum of electronic and thermal Free Energies=

$-540.753022$ 
II. Table SI1. Summary Data for Equation 6.

\begin{tabular}{|ccccccc|}
\hline $\begin{array}{c}\text { Conjugated Ion } \\
\text { Length }(\# \text { Cs) } \\
(\mathbf{1 6} \text { to } 16\end{array}$ & R1 & R2 & R3 & $\mathrm{R} 4$ & $\begin{array}{c}\Delta \mathrm{E} \\
\text { (kcal/mol) }\end{array}$ & $\begin{array}{c}\Delta \text { Chg/C } \\
(\%)\end{array}$ \\
3 to 5 & $\mathrm{Et}$ & $\mathrm{Et}$ & $\mathrm{nPr}$ & $\mathrm{nPr}$ & -15.8 & 6.3 \\
3 to 5 & $\mathrm{Et}$ & $\mathrm{Et}$ & $\mathrm{Et}$ & $\mathrm{Et}$ & -14.1 & 5.8 \\
3 to 5 & $\mathrm{Et}$ & $\mathrm{nPr}$ & $\mathrm{Et}$ & $\mathrm{nPr}$ & -13.6 & 5.4 \\
3 to 5 & $\mathrm{nPr}$ & $\mathrm{nPr}$ & $\mathrm{nPr}$ & $\mathrm{nPr}$ & -13.1 & 5.2 \\
3 to 5 & $\mathrm{nPr}$ & $\mathrm{nPr}$ & $\mathrm{Et}$ & $\mathrm{Et}$ & -11.4 & 4.7 \\
5 to 7 & $\mathrm{Et}$ & $\mathrm{Et}$ & $\mathrm{nPr}$ & $\mathrm{nPr}$ & -10.5 & 3.3 \\
5 to 7 & $\mathrm{Et}$ & $\mathrm{Et}$ & $\mathrm{Et}$ & $\mathrm{Et}$ & -9.3 & 3.0 \\
5 to 7 & $\mathrm{Et}$ & $\mathrm{nPr}$ & $\mathrm{Et}$ & $\mathrm{nPr}$ & -9.1 & 2.9 \\
5 to 7 & $\mathrm{nPr}$ & $\mathrm{nPr}$ & $\mathrm{nPr}$ & $\mathrm{nPr}$ & -8.8 & 2.8 \\
5 to 7 & $\mathrm{nPr}$ & $\mathrm{nPr}$ & $\mathrm{Et}$ & $\mathrm{Et}$ & -7.6 & 2.5 \\
7 to 9 & $\mathrm{Et}$ & $\mathrm{Et}$ & $\mathrm{nPr}$ & $\mathrm{nPr}$ & -7.6 & 2.1 \\
7 to 9 & $\mathrm{Et}$ & $\mathrm{Et}$ & $\mathrm{Et}$ & $\mathrm{Et}$ & -6.7 & 1.8 \\
7 to 9 & $\mathrm{Et}$ & $\mathrm{nPr}$ & $\mathrm{Et}$ & $\mathrm{nPr}$ & -6.6 & 1.9 \\
7 to 9 & $\mathrm{nPr}$ & $\mathrm{nPr}$ & $\mathrm{nPr}$ & $\mathrm{nPr}$ & -6.4 & 1.8 \\
7 to 9 & $\mathrm{nPr}$ & $\mathrm{nPr}$ & $\mathrm{Et}$ & $\mathrm{Et}$ & -5.5 & 1.5 \\
9 to 11 & $\mathrm{Et}$ & $\mathrm{Et}$ & $\mathrm{nPr}$ & $\mathrm{nPr}$ & -5.9 & 1.5 \\
9 to 11 & $\mathrm{Et}$ & $\mathrm{Et}$ & $\mathrm{Et}$ & $\mathrm{Et}$ & -5.1 & 1.3 \\
9 to 11 & $\mathrm{Et}$ & $\mathrm{nPr}$ & $\mathrm{Et}$ & $\mathrm{nPr}$ & -5.0 & 1.2 \\
9 to 11 & $\mathrm{nPr}$ & $\mathrm{nPr}$ & $\mathrm{nPr}$ & $\mathrm{nPr}$ & -5.0 & 1.2 \\
9 to 11 & $\mathrm{nPr}$ & $\mathrm{nPr}$ & $\mathrm{Et}$ & $\mathrm{Et}$ & -4.3 & 1.0 \\
\hline
\end{tabular}

Data correlation for "3 to 5": $\Delta \mathrm{E}=-2.60(\Delta \mathrm{Chg} / \mathrm{C})+0.66 ; \mathrm{R}^{2}=0.98$;

Data correlation for "7 to 9": $\Delta \mathrm{E}=-3.37(\Delta \mathrm{Chg} / \mathrm{C})-0.42 ; \mathrm{R}^{2}=0.95$. 